\title{
A Half Full Glass May Seem Fuller than a Half Emply One. Studies on the Influence of Regulatory Fit on Consumer Behavior
}

Citation for published version (APA):

Daryanto, A. (2008). A Half Full Glass May Seem Fuller than a Half Emply One. Studies on the Influence of Regulatory Fit on Consumer Behavior. [Doctoral Thesis, Maastricht University]. Datawyse /

Universitaire Pers Maastricht. https://doi.org/10.26481/dis.20080424ad

Document status and date:

Published: 01/01/2008

DOI:

10.26481/dis.20080424ad

Document Version:

Publisher's PDF, also known as Version of record

Please check the document version of this publication:

- A submitted manuscript is the version of the article upon submission and before peer-review. There can be important differences between the submitted version and the official published version of record.

People interested in the research are advised to contact the author for the final version of the publication, or visit the DOI to the publisher's website.

- The final author version and the galley proof are versions of the publication after peer review.

- The final published version features the final layout of the paper including the volume, issue and page numbers.

Link to publication

\footnotetext{
General rights rights.

- You may freely distribute the URL identifying the publication in the public portal. please follow below link for the End User Agreement:

www.umlib.nl/taverne-license

Take down policy

If you believe that this document breaches copyright please contact us at:

repository@maastrichtuniversity.nl

providing details and we will investigate your claim.
}

Copyright and moral rights for the publications made accessible in the public portal are retained by the authors and/or other copyright owners and it is a condition of accessing publications that users recognise and abide by the legal requirements associated with these

- Users may download and print one copy of any publication from the public portal for the purpose of private study or research.

- You may not further distribute the material or use it for any profit-making activity or commercial gain

If the publication is distributed under the terms of Article $25 \mathrm{fa}$ of the Dutch Copyright Act, indicated by the "Taverne" license above, 


\section{A Half Full Glass May Seem Fuller than a Half Empty One}

\section{Studies on the Influence of Regulatory Fit on Consumer Behavior}

Ahmad Daryanto 


\section{(C) 2008, Ahmad Daryanto, Maastricht}

All rights reserved. No part of this publication may be reprinted or utilized in any form or by any electronic, mechanical, or other means, now known or hereafter invented, including photocopying and recording or in any information storage or retrieval systems, without written permission of the copyright owner.

ISBN 9789052787114 :

Cover Design: Ara and Anto

Printed by: Datawyse Maastricht 



\title{
A Half Full Glass May Seem Fuller than a Half Empty One
}

\section{Studies on the Influence of Regulatory Fit on Consumer Behavior}

\author{
PROEFSCHRIFT \\ Ter verkrijging van de graad van doctor \\ aan de Universiteit Maastricht \\ op gezag van de Rector Magnificus Prof. Mr. G.P.M.F. Mols \\ volgens het besluit van het College van Decanen \\ in het openbaar ter verdedigen \\ op donderdag 24 april 2008 om 16.00 uur \\ door
}

Ahmad Daryanto 



\section{Promotores}

Prof. dr. J.C. de Ruyter

Prof. dr. M.G.M Wetzels

\section{Beoordelingscommissie}

Prof. dr. L.Borghans, voorzitter

Prof. dr. J.G.A.M. Lemmink

Prof. P.G. Patterson, School of Marketing, U. New South Wales, Australia 


\section{Lovingly dedicated to:}

Abak, Mirwan Apen, and Amak, Jusmihanum my wife, Ara,

and our beloved son, Ilham 



\section{ACKNOWLEDGEMENTS}

For the last four years, one of my biggest aspirations in life has been to obtain a $\mathrm{PhD}$ degree in Marketing. Although, this was supposed to be my own personal goal, achieving the final completion could not be realized without the help of many people. Therefore, I would like to express my sincere gratitude to them.

First of all, I would like to thank my first promotor Ko de Ruyter for giving me an opportunity to do a $\mathrm{PhD}$ under his supervision. I am indebted to Ko for his critical comments and feedback through many stimulating discussions that helped me to successfully complete this dissertation. His excellent writing skills assist me a lot in improving and repositioning my writing. Another special thank goes to my second promotor Martin Wetzels. Martin was a supportive and friendly supervisor. He is always a great resource for fulfilling my statistical inquiries. It is due to his influences that I shaped my interest in meta-analysis, a powerful statistical methodology that I used in the second chapter of this dissertation.

Furthermore, I would like to thank all members of my promotion committee for thorough reading of the manuscript and invaluable comments: Prof. dr. L. Borghans, Prof. dr. J.G.A.M Lemmink (Maastricht University), and Prof. P.G. Patterson (University of South Wales, Australia).

Special thanks also go to the members of the Department of Marketing at Maastricht University. In particular, I would like to thank Mirjam Schmidt and Marjoleine van Hout. I highly appreciate their kindness and help. Both had dedicated and acted as my family 'lawyer' when I had to appeal against incorrect decision of immigration office regarding a residence permit of my wife. I am grateful to Niek and Aga for proofreading the introduction and conclusion chapter of this dissertation and for being supportive office roommates.

Next, I would like to thank my 'family' and friends that made me feel at home in the Netherlands: Frenk and Kak Lena's family in Gulpen, Pak Muhammad and Uni Mirna's family in Geleen, Oma's family in de Heeg, Ruhuldah, Eva Susami, Bude Evi, and Hasanul and Nurul Hasanah's family when they were in Maastricht. My special thanks go to Bu Linda in Den Haag who helped me a lot from the very beginning of my stay here. My thank also goes to Bang Batara Simatupang DBA, Andri GW, and my other Indonesian friends in PPI-M and Pengajian KAMIL for creating an Indonesian atmosphere in Maastricht.

I also would like to thank my father and mother for their love and endless praying, and my big family in Padang and Lampung for their support.

Finally, my exceptional gratitude goes to my wife, Ara, and our beloved son Ilham, who have in many different ways inspired me and given my life a meaning. Ara, without you, I would not have been able to end this journey.

Above all, all praise and thanks be to Allah, who gives me strength, and whom I seek for protection. 


\section{TABLE OF CONTENTS}

CHAPTER 1: Introduction 1

CHAPTER 2: A Meta Analysis of the Regulatory Focus Fit Effect: Implica- 11 tions for Consumer Research

CHAPTER 3: Fitness by Fit: A Regulatory Fit Perspective of Reward 29

Preference in a Health Club Loyalty Program

CHAPTER 4: The Role of Regulatory Focus in Explaining Retailer's Attitude 51 and Behavior in relation to Channel Reward Programs

CHAPTER 5: Getting a Discount or Sharing the Cost? The Influence of Regu- 79

latory Focus Fit on Consumer Response to Private Health Insurance Policies

CHAPTER 6: Conclusion 99

$\begin{array}{ll}\text { APPENDICES } & 105\end{array}$

$\begin{array}{ll}\text { REFERENCES } & 109\end{array}$

$\begin{array}{ll}\text { ABOUT THE AUTHOR } & 124\end{array}$ 



\section{CHAPTER 1}

\section{Introduction}

Imagine Githa and Ade working out in a university gym. Githa, a master student, is preparing herself for the national university badminton tournament that is held annually in the summer break. Githa wants to increase her speed, endurance and get stronger. She always measures her muscle strength in every training and consults with her coach about her daily achievements. Ade is a $\mathrm{PhD}$ student in the same university. Working out in the gym, his goals are to manage his stress and to have a balance between mental effort and physical effort, between the intellectual labor required for his $\mathrm{PhD}$ and the exercise that keeps his body fit. He always makes sure that he does not miss any of his scheduled exercise sessions during the week. Furthermore, he never works out beyond the time he normally sets, because he wants to enjoy a healthy meal served in the gym canteen. These two people have different goals and different strategies in approaching their goals. Both receive a flyer from the gym management about the bonus provided to members if they work-out regularly in the gym for the coming twelve months. The flyers have two versions with different layouts. In one flyer Githa reads: "if you don't exercise in the gym at least two times per week from now on, you will fail to get the bonus of the year". In another flyer, Ade reads: "If you exercise in the gym at least two times per week from now on, you will get the bonus of the year".

The scenario illustrates above highlights two distinct goals in regulatory focus theory (Higgins, 1997) (i.e., Githa's goals that relates to her aspirations and Ade's goals of prevention and security) and two different ways a message is framed that Githa and Ade have read separately. This dissertation is about the fit between the goals and the way the messages are communicated and how its influences value of the goals attained and consumer behaviors. The research context of this dissertation is consumer behaviors in loyalty programs. In the next section, we present the controversy surrounding loyalty programs with respect to its effectiveness in creating value and building consumer loyalty. Then, we briefly discus the general concept of self-regulation, regulatory focus theory, and regulatory fit theory as a general theoretical framework utilized in this dissertation to explain how consumers create value. We also outlined the importance of regulatory fit research presented in a separate section. In the last sections, we present the objectives of the dissertation followed by the dissertation outline.

\section{Creating Value and Building Consumer Loyalty}

Creating value and building consumer loyalty are strategic objectives for firms operating in many of today's competitive industries. One manifestation of these objectives is the fact that loyalty programs are enjoying a rapidly growing popularity. As McKee (2007) reports, 
nearly $90 \%$ of American consumers participate in loyalty programs, with an average of four programs per consumer. These loyalty programs are ubiquitous across many industries such as airlines, hotels, banking, and retails. Furthermore, in B-to-B markets, these programs are also becoming popular, under the label of 'partnership programs' (Capizzi and Ferguson, 2005). Across both markets, these programs are developed and maintained on the premise that it is cheaper to keep existing customers than it is to attract new ones and that cultivating loyalty leads to company profitability (Reinartz and Kumar, 2000; Kivetz and Simonson 2002; Sirdeshmukh, Singh, and Sabol 2002). However, loyalty programs do not turn all customers into loyal customers or make the company the preferred supplier (Shugan, 2005; Leenheer et al., 2006). Moreover, many firms adopt these programs as a hype or 'me-too' scheme (Yi, 2007; Dowling, 2002). At the same time recent research has shown that such programs have a positive impact on consumers' share-of-wallet (Lewis, 2004; Wirtz, Mattila and Lwin, 2007). Thus, loyalty programs seem to send out a mixed message, which urges a need for a better understanding of consumer behavior in response to these programs. This dissertation aims at fulfilling this need.

Loyalty programs are basically value-enhancement programs as they are meant to increase consumers' value perceptions and customer retention. Indeed, enhanced value perception has been regarded as a determinant factor in fostering loyalty (e.g., Li, 2007; Yi and Jeon, 2003). While the key to enhancing value is understanding consumer needs and goals, little is known about how these factors affect the effectiveness of loyalty programs. The objective of this dissertation, thus, is to develop an insight into the role of factors that affect value perceptions and consumer responses to such programs. Specifically, we utilize regulatory focus (RF) theory (Higgins, 1997) as a theoretical foundation to explain value creation, as it provides a powerful framework for explaining how consumer goals drive their behavior. In the next section, we will briefly introduce regulatory focus theory as well as its theoretical roots. As this theory is a branch of the broad umbrella of self regulation theory, we start our introduction describing the general concept of self-regulation.

\section{The General Concept of Self-Regulation}

The explanation of the self-regulation concept may be best started by posing a number of illustrative questions; "why do some consumers consider more and various criteria when buying a product, whereas others consider only a few?", "why do some students study beyond course requirements to get an A (e.g., spend more time in library, approach their lecturer after class), whereas others just make sure that they just cover the required materials?" These types of questions have fascinated researchers in social psychology and consumer research more than a decade ago (Higgins, 1997). The answer to these questions can be related to the concept of self-regulation. This term has a connotation of regulation by the self, especially with regard to bringing the self into line with preferred standards (e.g. goals) (Vohs and Baumeister, 2004). Thus, self regulation encompasses people's efforts (i.e., in- 
volving both conscious and/or unconscious processes) to regulate their thoughts, emotions, and behaviors.

Nowadays, regulation research has addressed one of the most challenging topics related to human behavior across different sub-disciplines of psychology, ranging from social to consumer to developmental psychology. In general, researchers have relied on two approaches in studying self-regulation. The first approach emphasizes the importance of the self-monitoring process. This approach has been greatly influenced by cybernetic theory (from the Greek word of 'kybernetes', meaning "steersman"). Introduced by the well-known mathematician Norbert Wiener (1948) in the early $20^{\text {th }}$ century, this theory deals with the control of biological and mechanical systems. It shows how systems or objects can regulate themselves by making adjustments to conform their own programmed goals (e.g., a thermostat that controls a cooling and heating system to maintain a room's temperature). An important characteristic of this approach is the mechanism for a feedback process that allows the self-monitoring of the discrepancy between the current and the desired-end states or reference values. When there is a negative feedback (e.g., current temperature is below the desired level), the object reduces or eliminates the discrepancy between the current states and the reference value (i.e., the approach motives). In contrast, when there is a positive feedback (e.g., current temperature meets the desired level), the object attempts to avoid a discrepancy between the current states with the undesired end states or anti-goals (i.e., the avoidance motives). Research using this approach was initiated by an influential work of Carver and Scheier (1981) on affect self-regulation, which shows how people use their feelings as signals to detect a discrepancy between their current and the desired-end states (Carver and Scheier, 1981, 1998). The author's feedback-loop model was also called the TOTE (test-operate-test-exit) system comprising of four phases. In the first phase, i.e., the test phase, a person determines his current states (e.g., emotional state) and compares his current state with his desired-end state (e.g., preferred emotional state). The operate phase is initiated when a discrepancy between the current state and the preferred desired-end state is detected. This phase involves a process of change where actions commonly take places to move the self to the preferred desired-end states. In order to monitor the progress in the process of change, the test phase is again needed to compare the new current state as a result of change with the standard. When the discrepancy is not detected anymore, then the feedback-loop is terminated (i.e., exit phase). The theory of regulatory focus was originated form this goal-discrepancy model (Higgins, 1987). In the next section, we will explain how RF theory advances this theorizing.

The second approach in self-regulation research emphasizes the process of change, i.e., the operate phase in the TOTE system. In this approach, the focus of research is to understand how people' actions reduce or enlarge the discrepancy between people current and desired-end states. An example of a model using this approach is the self-regulatory strength model developed by Baumeister, Heatherton, and Tice (1994), which has lead to the development of self-regulatory resources theory. According to this theory, the core of 
self-regulation is the availability of internal self-regulatory resources enabling for selfcontrol. The self-regulatory resources encompass physical and psychological energies, such as willpower. Therefore, people's failures in self-regulation are maybe due to the lack or depletion in people self-regulatory resources. For example, it is easier for people to control their appetite when have just eaten than when they are hungry. This second approach views self-regulation as involving deliberate and conscious processing.

\section{Regulatory Focus Theory}

Almost a decade ago, a branch of self-regulation theory- the so-called 'regulatory focus theory' (RF) was developed (Higgins, 1997). The theory explains how consumers' motives to obtain certain goals affect their behavior. As mentioned in the previous section, RF theory originated from the theory of self-regulation toward desired-end states (e.g., goaldiscrepancy theory) (Carver and Scheier, 1981, 1998). However, the theory of selfregulation does neither distinguish different means of approaching end-states nor identify different types of desired-end state that relate to the different means of approach (Higgins, 1997). RF theory advances this theory by fulfilling these research gaps.

RF theory proposes that there are different ways of approaching different types of desiredend states. With respect to desired-end states, RF theory distinguishes two types of fundamental needs, namely nurturance (e.g., nourishment) and security (e.g., protection) and twotypes of desired-goals: ideal goals and ought goals. Ideal-goals are the goals that people would ideally like to achieve. These goals are typically the goals that are concerned with advancement, aspiration, and accomplishment (e.g., wanting a beautiful house). In contrast, ought-goals are the goals that people believe they ought to achieve. These goals are typically the goals that are concerned with duty, obligation, and responsibility (e.g., finishing a $\mathrm{PhD}$ dissertation within four years!). RF also distinguishes two different ways of approaching the desired-end states, i.e., using eagerness-related means (i.e., suitable means to use for approaching matches with the desired-end states) and vigilance-related means (i.e., suitable means to use for avoiding mismatches with the desired-end states). By means of illustration, let us assume that a desired end-state of a consumer is to have a digital camera. To approach this goal, he/she can extensively search for more information about the latest specifications, consider many criteria for product selection, and compare different products before purchasing, constituting the use of eagerness means. In contrast, for some consumers buying a digital camera is ensuring whether the digital camera is sophisticated enough to use (e.g., ensuring that it has a certain mega pixel resolution), which constitute the use of vigilance means. Thus, RF theory proposes that self-regulation in relation to nurturance needs and ideals goals is termed promotion focus, whereas self-regulation in relation to security needs and ought goals is termed prevention-focused. In goal pursuit, promotion-focused individuals prefer to use eagerness-related means because this is the type of means most suited to achieve ideal-goals. In contrast, prevention-focused individuals tend to use vigilance-related 
to achieve their ought-goals. In other words, using eagerness means is the natural strategy for promotion-focused individuals to reduce the discrepancy between current states and desired-end states, whereas using vigilance means for avoiding mismatches with desired end-states is the natural strategy for prevention-focused individuals.

Promotion and prevention foci are not bipolar constructs. An individual can be dominant in either focus, but also in both. Because there are two sub-regulatory focus systems in an individual, whereby one system may dominate the other, this influences how people perceive a certain goal. Therefore, a goal can be perceived as "ought" or "ideal", depending on which system is more active and dominant at the time of making a judgment. For example, for the goal of finishing a $\mathrm{PhD}$ within four years, some $\mathrm{PhD}$ candidates may perceive this goal as an obligation, whereas for others it may be an achievement (experience shows that it is very hard to finish on time!). Thus, the same glass can be either half-full of half empty, depending on the eyes of the beholder.

Regulatory focus is considered as a dispositional characteristic of an individual (i.e., traits) as well as a temporary, situationally-induced orientation (i.e., state variable). When approached as a trait variable, an individual's focus is assessed by using a regulatory focus scale, e.g., the self-guide strength measure (Higgins, Shah, and Friedman, 1997), or the promotion and prevention goals measure (Lockwood, Jordan, and Kunda, 2002). When operationalized as a state variable, regulatory focus is manipulated by framing an identical set of task involving as success or failure or as involving gain-nongain (promotion) or lossnonloss (prevention) (Crowe and Higgins, 1997).

Research on regulatory focus has revealed many distinct patterns of human behaviors associated with promotion and prevention regulations. Here, we mention several research findings across the three broad domains of psychology, i.e., affective, cognitive, and motivations. In the affective domain, for instance, RF has been used to predict individuals' emotional response to success and failures in relation to attaining or not attaining desired-end states (see Higgins, Shah and Friedman, 1997). Aforementioned authors have demonstrated that attaining ideal goals with promotion regulation produces cheerfulness-related emotions (e.g., happiness), whereas failing to attain ideal goals produces dejection-related emotions (e.g., disappointment). In contrast, attaining ought goals with prevention regulation produces quiescence-related emotions (e.g., relaxation), whereas failing to attain ought goals produces agitation-related emotions (e.g., nervousness). RF also has been shown to affect how people make a decision. For instance, research has demonstrated that promotionfocused individuals tend to generate more criteria and alternatives when making a purchase decisions rather than prevention-focused individuals (Crowe and Higgins, 1997). With regard to the application of RF theory in the motivation domain, RF research has uncovered individual's strategic behavior in goal pursuit (Forster, Higgins, and Bianco, 2003). It has been revealed that promotion-focused individuals emphasize speed at goal completion, whereas prevention-focused individuals pay more attention to the accuracy of tasks at hand. As a consequence, therefore, prevention-focused individuals are less likely to make errors. 


\section{Regulatory Fit Theory}

As has been explained in the aforementioned section, the natural tendency for promotionfocused individuals is to use eagerness-related means in pursuing a goal, whereas prevention-focused individuals tend to use vigilance-related means. RF theory proposes that the compatibility between the foci and the means used in goal pursuit results in the sense of feeling-right, which creates additional value (i.e., value-from-fit) independent of the value of the outcome of goal pursuit (Higgins, 2002). Thus, this theorizing expands RF theory to a so-called regulatory fit theory (Higgins, 2000, Avnet and Higgins, 2006). According to this theoretical extension, promotion-focused individuals who use eager means will value the goal process more than promotion-focused individuals who use vigilance means. In contrast, prevention-focused individuals who use vigilance means will value the goal process more than promotion-focused individuals who use vigilance means. For example, please consider again a consumer who wishes to buy a digital camera. For this consumer, having and enjoying a digital camera is valuable regardless of her/his foci and their ways (i.e., efforts) to buy the product. In addition to this outcome value, however, there is an additional value from fit. Therefore, a value of having a digital camera for promotion-focused consumers with eagerness means or prevention-focused consumers with vigilance means will be higher than those of promotion-focused consumers with vigilance means or preventionfocused consumers with eagerness means. This is because feeling-right is considered as relevant information to judge the outcome of decisions, which adds more value. Thus, value-from-fit seems to reflect a cultural maxim which specifies that "It is not enough to do good, one must do it the right way".

The phenomenon of value-from-fit creation has been explored by Higgins and his colleagues in series of recent studies (e.g., Higgins et al., 2003; Cesario, Grant and Higgins, 2004)). This research has revealed that value-from-fit occurs as a result of source confusion, i.e., a failure to discriminate the different sources of value associated with the process and associated with the outcome of goal pursuits. Because of this confusion, consumers who experience regulatory fit assign additional value to their digital camera, such that it is more valuable. However, when people were made aware of the 1 source of this feeling right sensation (e.g., by simply asking a question: "how do I feel about it?"), the fit effect diminished, as people do not use their feeling-right information anymore to judge the value of outcome decisions. This idea of source confusion account of value-from-fit can be traced back to the classic work of Schwartz and Clore (1996) on the effect of weather (sunny vs. rainy) on general life satisfaction. They found that weather influence people's evaluation of their life because of people' reliance on their moods as a relevant factor (e.g., people generally feel happy in a sunny day rather than in a rainy day). However, when people's attention was drawn to the weather as a source of their good or bad moods, the effect of weather on life satisfaction disappear because weather is not considered anymore as relevant factor to the judgment of life. 
Extant empirical research on regulatory fit has shown that the effect of fit can be transferred to consumer subsequent judgments. For instance, consumers assign higher value to objects of choice and more motivated to pursue their goals if they are experiencing regulatory fit (Higgins, Idson and Freitas, 2003). Since the birth of the regulatory fit theory, the identification of the regulatory fit effect continues to become a central research interest to further our understanding of the process of value creation in consumer decision making (Avnet and Higgins, 2006). This dissertation aims at improving this understanding.

\section{The Importance of Regulatory Fit Research}

Nowadays, research about consumers' regulatory focus and regulatory fit has become one of the central interests of consumer researchers because of its theoretical and practical significance. Its theoretical significance for consumer research lies in two important aspects. First, it is a key to understanding many different aspects of consumer behavior (e.g., self-control, motivation, emotion, judgment and decision-making), which 'takes a collective look at the various components influencing behavior (i.e., affective, cognitive, and behavioral factors) (Werth and Forster, 2007). For instance, the theory not only explains how a consumer will pursue his/her goals, but also predicts what type of emotions she/he will feel when she/he succeeds or fails to reach the goal (Higgins, 1997; Leone, Perugini and Bagozzi, 2005). Second, regulatory focus theory offers a new perspective in explaining underlying mechanisms affecting behaviors. In this case, regulatory focus is often proposed to refine existing theories used to explain the behavior. For instance, regulatory foci have been advanced as moderator variables in explaining framing effect (Aaker and Lee 2004), prospect theory (Kluger et al. 2004; Chernev 2004), and counterfactual thinking (Roese, Hur and Pennington, 1999). From a practical point of view, regulatory focus research helps policy makers (e.g., officials government), decision makers (e.g. company managers) in designing and formulating a better and more effective strategies (e.g., effective communication strategies) in order to encourage members of societies to adopt or not to adopt certain behaviors (e.g., buying a product, joining a program).

\section{Objectives}

The objective of this dissertation is to gain insight into the role of regulatory focus in affecting consumer value perception and subsequent behaviors such as investment decisions and consumer loyalty. As people behavior can be both influenced by their traits and external environment (Mischel, 2004), more specifically, in this dissertation, we study the impact of state and trait regulatory focus of consumers participating in loyalty programs. For this, we examine the impact of regulatory fit on their response to the communication of the elements of the program, i.e., program benefits. In order to successfully address the overall objective 
of this dissertation, we formulated four research projects. These projects were guided by the following objectives:

- To provide a quantitative synthesis of the research concerning the magnitude of the impact of regulatory fit.

- To empirically test the interaction effect between state regulatory focus and the way the reward is positioned on value perception and consumer subsequent behaviors.

- To assess the relative strength of the trait regulatory focus in studies that manipulates regulatory focus.

- To determine the relative impact of fit effect in comparison to context-specific determinants of variance in value perception.

\section{Dissertation Outline}

This dissertation comprises of four studies: one meta-analysis study reported in chapter 2 and three empirical studies described in chapter 3, 4, and 5 (see table 1). As each chapter constitutes a research paper on its own addressing specific research questions, therefore, they are independent but interrelated building blocks to answering the research questions.

Chapter 2 presents a meta-analytic study, which is a quantitative review integrating findings from extant research. The aim of this chapter is to assess systematic variation and strength of the regulatory fit effect. The importance of this study is to further our understanding of the process of value creation by identifying relevant systematic factors that influence the fit effect.

Chapter 3 reports studies examining consumers' reactions to loyalty program rewards. Two empirical studies are described in this chapter. The first study examines the interaction effect between the manner in which reward is positioned (addressing promotion or prevention concerns) and the way it gets communicated (gain or loss), which constitutes regulatory focus states. The second study examines the impact of regulatory and domainspecific traits on consumer reward choices. The research context of these studies is a health club loyalty program.

Chapter 4 presents two studies that examine the influence of regulatory focus on reward redemption decisions whereby the decisions involve risk and uncertainties with respect to type of rewards that can and can not be obtained and ROI or payoff of the rewards. The first study examines the interaction effect between regulatory focus and type of payoff. The second study examines the interaction effect between regulatory focus, type of payoff magnitudes, and type of payoff presentation formats. The context of the study is a channel reward program (CRP), which is a loyalty program initiated by a supplier for its retailer members.

Chapter 5 presents a study that investigates consumer's reactions to a health insurance policy, using regulatory focus as a theoretical basis. Specifically, this study examines the interaction effect between the manner in which a policy scheme is positioned and com- 
municated, according to gain or loss-framed information. In addition, this study assesses the role of perceived value as criterion variables that mediate the impact of fit effect on insurance purchase decisions.

Chapter 6 concludes with a synopsis of the main findings about the role of regulatory focus in affecting consumer responses toward company-initiatives program aiming at building consumer loyalty. Implications and future research are also discussed. 


\begin{tabular}{|c|c|c|c|c|}
\hline & Conceptual focus & Research Context & Methodology & Analysis \\
\hline Chapter 2 & Meta-analysis of the impact of fit effect & Literature study & $\begin{array}{l}\text { Meta-regression analy- } \\
\text { sis }\end{array}$ & SPSS using Meta-Analysis macro \\
\hline Chapter 3 & $\begin{array}{l}\text { (1) Fit effect on exercise intention, perceived } \\
\text { value, and loyalty, 2) mediation effect of per- } \\
\text { ceived value. }\end{array}$ & $\begin{array}{l}\text { Health club loyalty } \\
\text { program }\end{array}$ & $\begin{array}{l}\text { Experimental design } \\
\text { with survey, Survey }\end{array}$ & $\begin{array}{l}\text { Structural Equation Modeling } \\
\text { (Mplus), Partial Least Squares } \\
\text { (PLSGraph) }\end{array}$ \\
\hline Chapter 4 & $\begin{array}{l}\text { (1) Fit effect on perceived value and redemption } \\
\text { decisions; (2) Regulatory focus on processing } \\
\text { uncertainty information. }\end{array}$ & Retail loyal program & $\begin{array}{l}\text { Experimental } \\
\text { with survey }\end{array}$ & Partial Least Squares (PLSGraph) \\
\hline Chapter 5 & $\begin{array}{l}\text { (1) Fit effect on perceived value, purchase inten- } \\
\text { tion, (2) Mediation effect of perceived value, (3) } \\
\text { relative impact of fit effect in comparison with the } \\
\text { strength of domain-specific constructs. }\end{array}$ & $\begin{array}{l}\text { Health insurance pro- } \\
\text { gram }\end{array}$ & $\begin{array}{l}\text { Experimental } \\
\text { with survey }\end{array}$ & Partial Least Squares (PLSGraph) \\
\hline
\end{tabular}




\section{CHAPTER 2}

\section{A Meta Analysis of the Regulatory Fit Effect:}

\section{Implications for Consumer Research}

We conducted a meta-analytic study of previous regulatory focus research to assess systematic variation and strength of the regulatory fit effect. The magnitude of the fit effect is assessed on the basis of the size of the interaction term between the regulatory focus operationalization and a range of variables. Specifically, we examine; (1) feeling-right operationalization, (2) type of regulatory focus, (3) research domain, (4) use of incentives, (5) type of student sample and (6) participants' cultural background. We employ meta-regression analysis (MRA) to assess the association between these factors and effect size heterogeneity. Implications for consumer research and limitations of the study are addressed.

This chapter is largely based on: Daryanto, Ahmad, Ko de Ruyter and Martin Wetzels. A Meta Analysis of Regulatory Fit Effect: Implications for Consumer Research. To be submitted for a $2^{\text {nd }}$ submission at the Journal of Consumer Research. 


\section{Introduction}

A popular as well as fascinating topic in current consumer research is the impact of regulatory focus (RF) on emotions, judgments and behaviors (Higgins 1997). RF theory proposes that consumers differ in how they present and experience basic needs, how they act in goal pursuit and how they value outcomes (Molden, Lee and Higgins, 2007). The theory discerns two distinct foci; a promotion and prevention focus. The promotion focus is related to nurturance needs, advancement and progress, and gain versus non-gain outcomes. In contrast, the prevention focus is associated with security needs, duty, obligations and responsibility, as well as loss versus no-loss outcomes.

Promotion and prevention foci have been regarded as both chronic predispositions of individuals (i.e., traits) and states that can be momentarily induced by either a situation or task (Crowe and Higgins, 1997; Higgins 1997). Previous studies have assessed regulatory foci in relation to the effect of incentives on task-related performance (Shah, Higgins, and Friedman 1998), motivations and goals (Förster el. al 2001; Freitas and Higgins 2002; Freitas, Liberman and Higgins 2002), persuasion (Aaker and Lee 2001; Cesario, Grant and Higgins 2004) and emotions (Brockner and Higgins 2001). For instance, Shah, Friedman, and Higgins (1998) find that the performance of promotion-focused individuals is higher when incentives are framed in terms of accomplishments, whereas the performance of prevention-focused individuals is higher in case incentives are framed in terms of safety.

Recently, RF theory has branched out to include the concept of regulatory fit (Avnet and Higgins 2006; Kruglanski 2006). This fit occurs when the manner in which a decision is made sustains one's regulatory orientation. Preliminary empirical testing reveals that when the fit effect occurs; (1) consumers feel right about the pursuit of their goals (e.g. Cesario, Grant and Higgins 2004) and (2) their evaluative response toward objects is strengthened (Avnet and Higgins 2006). As a result, fit creates value (commonly known as 'value-fromfit') that can be transferred to subsequent judgments and behavior. For instance, Freitas and Higgins (2002) show that in case consumers assign higher value to objects of choice, they are more motivated and pursue more goals. This has lead Avnet and Higgins (2006) to conclude that the identification of the regulatory fit effect is crucial to further our understanding of the process of value creation in consumer decision making.

However, this claim does not seem to be fully substantiated by (recent) empirical evidence. So far, no study has provided an in-depth assessment of the magnitude of the impact of the fit effect. For example, while Higgins et al. (2003) report that the effect of fit on value is relatively high for participants who experience fit compared to those in non-fit conditions (i.e., between 40-60\% higher), Cesario et al. (2004) report an impact of fit on value that is considerably lower (i.e., around $12 \%$ ). There seems to be a paucity of generalizable research on the impact of regulatory fit. Thus, we seek to clarify the role of regulatory fit in consumer decision-making by providing a quantitative synthesis of the research 
evidence concerning the magnitude of the impact of regulatory fit, review progress in testing this dominant effect and highlight research avenues for enriching our understanding of this important phenomenon.

In the emerging literature on regulatory fit, explanations for diverging results with regards to the fit effect are scarce. For instance, Avnet and Higgins (2006) argue that the size of the fit effect may vary with respect to the types of regulatory focus (i.e., trait and state). Alternatively, narrative explanations have been offered with respect to the fact that the fit effect may vary across studies due to variation in specific study characteristics (e.g., Briely and Aaker 2006; Wang and Lee 2006). Therefore, our aim is to assess quantitatively whether relevant systematic factors influence the magnitude of the fit effect. Specifically, we examine the relationship between the estimate of the size of fit effect and the following study variations; (1) feeling-right operationalization, (2) type of regulatory focus, (3) research domain, (4) the use of incentives, (5) type of student sample, and (6) participants' cultural background. We employ meta-regression analysis (MRA) as this enables us to take into account the heterogeneity in estimate across studies in relation to aforementioned moderators.

\section{Prevalent Study Variations}

\section{Feeling-Right Operationalization}

Regulatory fit creates value that results from a feeling-right-experience. Researchers have used two different ways of operationalizing this experience (Aaker and Lee 2006); (1) a process-based approach and (2) an outcome-based approach (also called a regulatory relevance approach). In studies that use a process-based approach, participants are asked to adopt specific strategies that are consistent with their regulatory orientations in performing a specific task. These strategies are eagerness strategies (e.g., approaching matches with endstates) for promotion-focused individuals and vigilant strategies (e.g., avoiding mismatches with end-states) for prevention-focused individuals. For instance, Freitas and Higgins (2002) create a fit effect by presenting student participants with strategies to earn high grades. A fit effect occurs when promotion-focused students are prompted with eagerness strategies, such as attending all classes and spending more time in the library. Alternatively, when prevention-focused students are prompted with vigilant strategies, such as avoiding missing classes and spending less time at parties, a fit effect is also found. In studies that use an outcome-based approach, participants are prompted with outcomes to which promotion or prevention-focused participants are sensitive. For example, Semin et al. (2005) create a fit effect by presenting participants with benefits of physical exercise. A fit effect occurs when promotion-focused participants are informed about abstract benefits of exercise (e.g., feeling more energetic) and when prevention-focused participants are confronted with con- 
crete benefits (e.g., burning an x amount of calories per hour). However, the impact of outcome-based and process-based regulatory fit appears to diverge within and across recent studies (Aaker and Lee 2001; Semin et al. 2005; Aaker and Lee 2006; Avnet and Higgins 2006). Our meta-analytic approach is intended to yield more insight as to whether the manner of feeling-right operationalization is a systematic source of variance of effect size across studies.

\section{Type of Regulatory Focus}

The fit effect is a function of an individual's regulatory focus, which has been used either as a state or trait variable. The rationale for this is based on the general principle that personal orientations can both be conceptualized as a cross-person source of variability as well as a situational source of variability, having similar effects on motivation and behavior (Higgins 2000). Past research has routinely assessed the impact of trait and state RF on the same response measures with the aim of examining whether the effects of both types of RF on these response measures are similar. As a trait variable, promotion or prevention foci are commonly assessed by means of self- reported measures (e.g., Lockwood, Jordan and Kunda (2002) or measures of trait accessibility, i.e., a self-guide strength measure based on response latency (e.g., Higgins et al. 1994). As a state variable, a variety of methods have been used to induce individuals' promotion or prevention foci. While some researchers argue that situationally-induced RF cancels out the effect of trait-like RF (Higgins 2000; Higgins et al. 2003), others adhering to, for instance, the interactionist perspective (Steyer et al. 1999; Mischel 1999) hold that personal traits add variability to experimental manipulations. So far, however, no study has undertaken a simultaneous approach so that empirical evidence as to the prevalence of one over the other is lacking. Therefore, we examine the impact of both types of regulatory focus in our meta-analysis.

In order to examine the RF trait-state relationships, we assess effect size variation in relation to trait and four types of RF inducements; (1) ideal-ought goal priming (e.g., Higgins et al. 1986; Liberman et al. 1999), (2) identical task priming (e.g., Forster et al. 1998), (3) approach-avoidance strategic priming (e.g., Higgins et al. 1994; Semin et al. 2005), and (4) attribute priming (e.g., Spiegel et al. 2004; Aaker and Lee 2001).

\section{Research Domain}

Commonly, regulatory focus research is conducted in a specific research domain. Prevalent types of domain are personal health (e.g., studies on nutritional habits by Cesario et al., 2004) and education (e.g., studies on students' academic motivations by Freitas and Higgins 2002). In addition, there is also a large contingent of studies that have remained domainneutral, focusing on cognitive processing by means of laboratory tasks (e.g. puzzle- and 
anagram-solving studies by Freitas and Higgins 2002). In domain-specific RF research, participants are usually instructed to read vivid descriptions of a scenario. In the laboratory task setting, participants are instructed to work on a certain problem-solving task. Working on these tasks requires more cognitive effort, attention focus and, consequently, more systematic processing on the part of participants. As recent theorizing on RF suggests, the occurrence of the fit effect is indicative of heuristic processing, rather than systematic processing (Aaker and Lee 2006). Accordingly, the use of the regulatory fit in task-solving studies could be expected to be smaller relative to domain-specific RF studies. At the same time, Idson et al (2001) report that the motivations of participants tend to be higher when they experience regulatory fit. Therefore, it seems important to systematically assess the impact of the research setting on the size of the regulatory fit effect in our meta-analysis.

\section{The Use of Incentives}

Recent research on RF shows that a fit effect is more likely to occur when individuals are not explicitly motivated to process information and/or their level of involvement in the situation is low (Aaker and Lee 2006; Wang and Lee 2006). In this case respondents are more likely to use heuristic information processing, relying on perceptual salience of information, such as product attributes. The use of heuristic strategies also increases the chance that respondents are unaware of the source of regulatory fit (see Higgins et al. 2003; Cesario, Grant and Higgins 2004).

Frequently, however, experimental RF studies use a variety of incentives, such as money, course credits and personal gifts in order to increase the motivation to participate and/or participants' involvement. Past studies (e.g. Martin and Marcuse 1958; Sharp et al. 2006) have shown that motivations of participants who are offered incentives and those who participate voluntarily differ. Furthermore, as the degree of heuristic processing decreases, it will be likely that the use of incentives may have a negative impact on the size of the fit effect. However, as this issue has not been comprehensively addressed in the RF literature so far, we examine the impact of the use of incentives. More specifically, we assess effect size variation in relation to the aforementioned types of incentives and voluntarily participation.

\section{Type of Student Sample}

The appropriateness of using student sample as a representative of "real consumers" has been a subject of controversial debate in consumer research. Results from a second order meta-analysis of social science research (Peterson 2001) suggest that studies that employ a homogeneous sample (e.g., college students) may yield results that differ from those that use non-homogenous samples (e.g., the general public). As a consequence, it is recom- 
mended that this sample characteristic should be "routinely incorporated in consumer behavior studies as covariates to analytically assess their potential effects" (Peterson 2001, p.459). Except for a few notable exceptions (i.e., Leung and Lam 2003; Zhou and Pham 2004), the large majority of regulatory focus studies use either middle/high school or college student samples. Little is known about the fact whether the use of a particular type of student sample has a diverging impact on the fit effect. College students are more likely to have more highly developed cognitive structures as well as a higher need for cognition which in turn may be more conducive to engaging in effortful cognitive activities relative to high school students. There is evidence that individuals who have a higher need for cognition tend to use more systematic processing style, whereas individuals with a lower need for cognition are more likely to engage in heuristic processing (Cacioppo et al., 1996). As mentioned in the previous section, the size of the regulatory fit effect is related to an individual's strategy for processing information, such that a heuristics information processing style increases the likelihood and chance of fit (Avnet and Higgins 2006). Therefore, it seems relevant to take this major study variation into account in a meta analysis of the regulatory fit effect.

\section{Participants’ Cultural Background}

Previous research has revealed that the role that RF plays in consumer decision-making is dependent on aspects of culture. Specifically, Aaker and Lee (2001) contend that the individualism-collectivism dimension of culture (cf. Hofstede 1980) influences the impact of RF. In general, collectivistic cultures are more closely associated with a prevention focus, as these cultures encourage the values of obligation and reciprocity. In contrast, in individualistic cultures a promotion focus is likely to be more prevalent, as the values of progress and advancement are central to this cultural background (Aaker and Lee 2001). Nevertheless, recent research shows that the influence of regulatory foci is not always robust across cultures and that the size of the fit effect may more strongly depend on other study characteristics, such as information processing and incentives (Briely and Aaker 2006). Thus, it seems warranted to systematically assess the impact of cultural background of the regulatory fit effect by means of a meta analysis, which will be introduced in the next section.

\section{Methodology}

\section{Study Selection}

The papers included in the meta analysis were identified using several keywords: regulatory focus, regulatory goal, self-regulatory focus, self-regulation, and regulatory fit. We 
searched for papers in the several electronic scientific databases, such as ABI/INFORM, EBSCO, Proquest, Google Scholar and Scirus. Studies selected in the sample had to meet the following criteria. First, we checked whether the regulatory fit was examined by employing one of the aforementioned four types of RF inducement (ideal-ought goal priming, identical task priming, strategic priming, and attribute priming) and the process-based and/or outcome-based approach to the operationalization of feeling right. As a result, those regulatory fit effects created from locomotion and assessment orientation (e.g., Kruglanski et al.2000; Avnet and Higgins 2003) were excluded. Second, research articles needed to provide adequate statistics for calculating effect sizes (i.e., an F test with 1 degree of freedom, $\mathrm{t}$ statistics, and $\chi^{2}$ value with 1 degree of freedom for calculating the partial eta squared). Therefore, studies that did not meet this criterion (or yielded the information after email communication with lead authors) and hence contained insufficient information for computing effect sizes were excluded from the analysis. A third criterion that we employed was publication in English-language, international academic journals. Furthermore, a number of regulatory fit studies report regulatory fit effects on mood, i.e. participants' feelings while making a decision. In the present meta analysis, we excluded effect sizes associated with such mood measures. The reason is that fit effects on mood are universally reported as insignificant and mood measures were commonly treated as covariates (Avnet and Higgins 2006; Higgins et al. 2003). In fact, one of the rare systematic findings in RF theory so far is the fact that participants' affective states during decision-making are independent of the regulatory fit effect (Higgins et al.2003, Higgins 2005). Finally, some studies did report that the interaction effect is not significant. For instance, Kim (2006) found no interaction effect between regulatory focus and framing on the social risks with respect to smoking). Such effect sizes are assumed to have a value of zero (Rosenthal 1991) and were included in the analysis.

\section{Calculating Effect Sizes}

To compare the interaction effect across studies, we needed a common measure of effect size. We used partial eta squared as this can be converted to $r_{\text {contrast }}$, which is a specific kind of association measure (see Rosenthal et al. 2000; Hullet and Levine 2003). Partial eta squared is defined as the proportion variance in the dependent variable accounted for by the independent variable divided by the amount of variance in the dependent variable that is unexplained by any other systematic factors in the design (i.e., after these other systematic factors have been partialled out (Cohen 1973)). The rationale for the partialling out is that because these systematic factors (e.g., any experimental variable) may induce additional variance in the dependent measures that makes comparison of an intended effect size across studies became unreasonable (Cohen 1973; Hullet and Levine 2003; Hunter and Schmidt 1999). Finally, the rationale for the conversion of partial eta squared to the $r$ family effect is 
that the effect size of $\mathrm{r}$ can be transformed to a Fisher- $\mathrm{Z}$ transformation of $\mathrm{r}$ in order to normalize the distribution.

The $r_{\text {contrast }}$ is analog to partial correlation in regression analysis (Rosenthal et al. 2000; Rosenthal and DiMatteo 2001). Therefore, the squared of $r_{\text {contrast }}$ refers to the proportion of variation in the dependent measure explained by the contrast, after variation due to other contrasts have been partialled out. Thus, the value for $r_{\text {contrast }}$ can be obtained by taking the square root of partial eta squared (see Hullet and Levine 2003) or calculated directly by using a significant $\mathrm{F}$ statistic of ANOVA with 1 degree of freedom, a t-statistic, and a $\chi^{2}$ statistics with 1 degree of freedom (see Rosenthal et al. 2000; Hullet and Levine 2003).

Some studies may have several response measures that were averaged or summed to make a composite index. Measurement error can occur as a result of imperfect measurements that attenuate the correlation between them. This will systematically lower the $r_{\text {contrast }}$. Following Hunter and Schmidt (2004), we correct for this attenuation. The formula of Hunter and Schmidt (2004) for attenuation correction for correlation coefficient $\left(\mathrm{r}_{\mathrm{c}}\right)$ between variable of $\mathrm{x}$ and $\mathrm{y}$, is:

$$
r_{c}=\frac{r_{x y}}{\sqrt{r_{x x}} \sqrt{r_{y y}}}
$$

where $r_{x x}$ and $r_{y y}$ is the measurement variability of variable $\mathrm{x}$ and $\mathrm{y}$, respectively. We adapt this formula to our case by setting $r_{x y}$ equals to $r_{\text {contrast }}$ and $r_{x x}$ to 1 . Fortunately, studies often reported coefficient reliability, i.e., Cronbach's coefficient alpha that can be used as an estimator for dependent measures of $r_{y y}$. In the case of studies that uses judges, effective reliability is computed as a substitute for Cronbach's alpha by using the Spearman-Brown formula (see Stajkovic 1999):

$$
R=\frac{n r}{1+(n-1) r}
$$

where $R$ = effective reliability, $n=$ number of judges, and $r=$ mean interrater reliability.

\section{Combining Effect Sizes}

As noted in the aforementioned section, because of the non-normality of the $r$ sampling distribution, we needed to normalize the distribution by transforming $r_{\text {contrast }}$ using a Fisher's Z-transformation (zr) (Hedges and Olkin 1985; Rosenthal and DiMatteo 2001). Because the expected value of $r_{\text {contrast }}$ may be slightly biased (i.e., underestimation of population $r_{\text {contrast }}$ ) due to a small sample size or when the true population value is in the range of 0.4-0.6 (Hedges and Olkin 1985), it may be reduced by computing the unbiased version of $r_{\text {contrast }}$ 
$\left(\mathrm{r}^{*}=r_{\text {contrast }}+\left[r_{\text {contrast }}\left(1-r_{\text {contrast }}{ }^{2}\right) / 2(\mathrm{n}-3)\right]\right.$. Next, the standard Fisher's Z-transformation is applied: $\mathrm{Zr}^{*}=.5 \log \left[\left(1+r^{*}\right) /\left(1-/ \mathrm{r}^{*}\right)\right]$. The sampling error variance for $\mathrm{Zr}^{*}$ is closely approximated by $1 /(n-3)$, where $n$ is the sample size. Note that some researchers advocate the use of untransformed effect size (e.g. procedure advocated by Hunter and Schmidt 1990) if the sample size is quite large because the standard errors of the estimate, confidence intervals and homogeneity tests can be different (Shadish and Haddock in Cooper and Hedges 1994). For interpretation purposes, the estimates computed using $\mathrm{Zr}^{*}$ were transformed back into $r_{\text {contrast }}$ (i.e. $r_{\text {contrast }}=\left(e^{2 Z r^{*}}-1\right) /\left(e^{2 Z r^{*}}+1\right)$, and then, to partial eta-squared.

\section{Meta-regression Analysis}

Having obtained a common effect size across studies, we performed a homogeneity test to see whether each set of effect sizes (i.e., zr) shared a common effect sizes (Hedges and Olkin 1985). We calculated Cochran's $\chi^{2}$ statistics where homogeneity has an approximate $\chi^{2}$ distribution with degrees of freedom equal to the number of effects sizes minus 1 (Hedges and Olkin, 1985). If the test is found to be significant, i.e., indicating a systematic variability in the effect sizes, a moderator variable analysis is warranted to identify sources of heterogeneity.

Subsequently, we explored the sources of heterogeneity by conducting a random effects meta-regression analysis that relates the effect size statistics to potential moderators, i.e., levels of major study characteristics. The advantage of using such an approach is that it focuses on differences between rather than within levels of study characteristics. Therefore, this method of analysis is appropriate to use to explore the contribution of each moderator in affecting the heterogeneity even if an initial overall Cochran's $\chi^{2}$ (or Q-test) is insignificant. Furthermore, it also allows for estimating residual heterogeneity that is not explained by potential moderators (Thompson and Higgins 2002). Finally, in a random setting, inferences from the results of the study are generalizable beyond the set of studies in the present sample (Hedges and Vevea 1998, Hunter and Schmidt 2000).

We used the SPSS Macro of Lipsey and Wilson (2001) with a restricted maximum likelihood (REML) estimator to estimate the regression parameters, their standard errors, and the residual heterogeneity variances. In this procedure, each effect sizes is weighted by its inverse within-study variance and between-study variance (Thompson and Higgins 2002).

Following Longi, Nijkamp and Poot (2005) we conducted a sensitivity analysis by estimating a model after weighting effect size by a measure of study quality. Sensitivity analysis is needed to examine the relative importance of study quality in influencing the estimated effect of certain study characteristics on effect sizes. Unlike Longi, Nijkamp and Poot (2005) who use dummy variables for subjectively differentiating between well respected and less respected academic journal qualities, we introduced a novel approach that 
uses the ISI (Thomson Institute for Scientific Information) impact factor for journal quality. Despite its limitations (among which are ignoring differences between (sub)disciplines, the questionable correlation between the quality of one particular paper and the journal in which it appears and the occurrence of self-citations), ISI impact factors are gaining wide popularity as an indicator of journal quality (McGregor 2007). We weighted effect sizes by ISI journal impact factors as well as by multiplying the impact factor with the inverse of within and between study variance (i.e. combining subjective assessment with objective quality).

\section{Results}

\section{Descriptive Statistics}

We obtained 83 effect sizes from 20 research articles (see Table 2.1).

As shown in Table 2.1, the articles were published between 1999 and 2007. The majority of the articles provided more than one effect size. Occasionally, multiple effect sizes came from the same study (i.e., multiple experiments) that report different response measures (e.g., Kim 2006). Some researchers argue that treating these effect sizes as statistically independent can cause a bias in the analysis (i.e., due to a correlation between response measures) and suggest reducing these effect sizes into a single composite measure (e.g., by averaging) (see Rosenthal and Rubin 1986) or taking into account the nested structure of the data (e.g., Kalaian and Raudenbush 1996; Bijmolt and Pieters 2001). Another approach is to treat effect sizes independently by fitting meta-analytic models with a Generalized Least Squares (GLS) estimator (e.g., Longi et al. 2005). In this study, we opted for the latter effect sizes independently to avoid information loss and estimate the model with an REML estimator, taking into account within and between study variance as well as the quality of studies. The summary statistics of the overall effect sizes are shown in 2.2. 
Table 2.1: Number of Effect Sizes per Study

\begin{tabular}{lllll}
\hline & & & Number \\
ID & Authors & Year & Journal* & Effect size \\
\hline 1 & Kirmani and Zhu & 2007 & JMR & 6 \\
3 & Zhao and Pechman & 2007 & JMR & 4 \\
4 & Werth and Forster & 2007 & EJSP & 9 \\
5 & Keller & 2006 & JCR & 3 \\
6 & Avnet and Higgins & 2006 & JMR & 1 \\
7 & Florack and Scarabis & 2006 & PM & 1 \\
8 & Jain et al. & 2006 & JCR & 4 \\
9 & Kim & 2006 & JA & 7 \\
10 & Wang and Lee & 2006 & JMR & 3 \\
11 & Forster and Higgins & 2005 & PS & 1 \\
12 & Semin et al. & 2005 & JPSP & 6 \\
13 & Louro et al. & 2005 & JCR & 6 \\
14 & Cesario et al. & 2004 & JPSP & 4 \\
15 & Lee and Aaker & 2004 & JPSP & 14 \\
16 & Idson et al. & 2004 & PSPB & 1 \\
17 & Higgins et al. & 2003 & JPSP & 1 \\
18 & Freitas and Higgins & 2002 & PS & 5 \\
19 & Forster el al & 2001 & JESP & 1 \\
20 & Higgins et al. & 2001 & EJSP & 1 \\
21 & Roese et al & 1999 & JPSP & 1 \\
22 & Forster et al. & 1998 & JPSP & 4 \\
& & & Total & 83 \\
\hline
\end{tabular}

* JMR=Journal of Marketing Research, JCR=Journal of Consumer Research, $\mathrm{PM}=$ Psychology Marketing, JA=Journal of Advertising, PS=Psychological Science, JPSP $=$ Journal of Personality and Social Psychology, PSPB $=$ Personality and Social Psychology Bulletin, JESP=Journal of Experimental Social Psychology, EJSP= European Journal of Social Psychology. 
Table 2.2: Statistical Summary of Interaction Effect Size

\begin{tabular}{|c|c|c|c|}
\hline \multicolumn{2}{|l|}{$\mathrm{N}$} & \multicolumn{2}{|l|}{83} \\
\hline \multirow[t]{3}{*}{ Mean } & (unweighted) & 0.332 & (Fisher-Z transform, zrc) \\
\hline & & 0.103 & (Partial-eta squared) \\
\hline & & 0.321 & $\left(\mathrm{r}_{\text {contrast }}\right)$ \\
\hline Min & $(\mathrm{zrc})$ & 0 & \\
\hline Max & $(\mathrm{zrc})$ & 1.006 & \\
\hline \multicolumn{2}{|c|}{ Proportion $r$-contrast $<0.1$} & $13 \%$ & \\
\hline \multicolumn{2}{|c|}{ Proportion $0.1<=$ r-contrast $<0.3$} & $13 \%$ & \\
\hline \multicolumn{2}{|c|}{ Proportion $0.3<=$ r-contrast $<0.5$} & $57 \%$ & \\
\hline \multicolumn{2}{|c|}{ Proportion $\mathrm{r}_{\text {contrast }}>=0.5$} & $17 \%$ & \\
\hline \multicolumn{4}{|c|}{$\mathrm{Q}(83)=287.576, \mathrm{p}<0.001$} \\
\hline \multirow[t]{2}{*}{ Estimate } & Random Effect (zrc) * & 0.267 & $\mathrm{R} 2=0.451$ \\
\hline & Random Effect (zrc) ** & 0.317 & $\mathrm{R} 2=0.461$ \\
\hline
\end{tabular}

* effect sizes were weighted by inverse within-study variance; ${ }^{* *}$ effect sizes were weighted by inverse within-study variance, between-study variance and study quality

As shown in the table, the unweighted mean effect size of the Fisher-Z transformation is 0.332, which is equivalent to an r-contrast of 0.321. According to Cohen (1988), this would suggest that the strength of the fit effect is moderate. However, taking the overall effect sizes into account, $17 \%$ can be considered as substantial. Based on Shadish and Haddock (1994), the Q-test for heterogeneity on effect sizes of this sample is 287.576, which is highly significant ( $<00.00, \chi^{2}$ with 82 degree of freedom). This is an indication that the true effect sizes are not equal across sample sizes in our meta study and that there may be a systematic variation that can be accounted for by potential moderators. Therefore, we conducted a random effect meta-regression with potential categorical moderators, as discussed in the previous section. The mean effect size estimated with random effect are displayed in the two last rows of Table 2.2 and the random effect meta-regression results are shown in Table 2.3. 
Table 2.3: Meta-regression results

\begin{tabular}{|c|c|c|c|}
\hline $\begin{array}{c}\text { (1) } \\
\text { Moderator }\end{array}$ & $\begin{array}{c}(2) \\
\text { Categories }\end{array}$ & $\begin{array}{c}\text { (3) } \\
\text { REML }^{n}\end{array}$ & $\begin{array}{c}\text { (4) } \\
\text { REML }^{\wedge}\end{array}$ \\
\hline \multirow[t]{3}{*}{ Feeling-right operationalization } & Process-based $^{b}$ & - & - \\
\hline & Outcome-based & -0.084 & -0.093 \\
\hline & & $(0.059)$ & $(0.063)$ \\
\hline \multirow[t]{9}{*}{ Type of Regulatory Focus } & Trait $^{b}$ & - & - \\
\hline & Ideal-ought & $-0.225^{*}$ & $-0.234 * *$ \\
\hline & & $(0.084)$ & $(0.085)$ \\
\hline & Identical tasks & $-0.306^{* * *}$ & $-0.306^{* * *}$ \\
\hline & & $(0.072)$ & $(0.071)$ \\
\hline & Approach/avoidance & $-0.349 * * *$ & $-0.349 * * *$ \\
\hline & & $(0.073)$ & $(0.075)$ \\
\hline & Attribute & $-0.122 *$ & $-0.125 * * *$ \\
\hline & & $(0.071)$ & $(0.072)$ \\
\hline \multirow[t]{7}{*}{ Research Domain } & Laboratory task $^{\mathrm{b}}$ & - & - \\
\hline & Other & $-0.173 *$ & $-0.173 *$ \\
\hline & & $(0.085)$ & $(0.085)$ \\
\hline & Health & $-0.127^{*}$ & $-0.113 *$ \\
\hline & & $(0.074)$ & $(0.073)$ \\
\hline & Education & $-0.351 * * *$ & $-0.358 * * *$ \\
\hline & & $(0.102)$ & $(0.102)$ \\
\hline \multirow[t]{7}{*}{ The Use of Incentive } & Voluntarily $^{b}$ & - & - \\
\hline & Monetary & $-0.235 * *$ & $-0.231 * *$ \\
\hline & & $(0.0807)$ & $(0.082)$ \\
\hline & Course credit & $-0.226^{*}$ & $-0.239 * *$ \\
\hline & & $(0.089)$ & $(0.090)$ \\
\hline & Personal gift & -0.059 & -0.059 \\
\hline & & $(0.096)$ & $(0.119)$ \\
\hline \multirow[t]{3}{*}{ Type of Student Sample } & High school ${ }^{b}$ & - & - \\
\hline & College & $0.280 * *$ & $0.293 * *$ \\
\hline & & $(0.095)$ & $(0.097)$ \\
\hline \multirow[t]{3}{*}{ Cultural Background } & Independent $^{b}$ & - & - \\
\hline & Interdependent & 0.092 & 0.098 \\
\hline & & $(0.112)$ & $(0.133)$ \\
\hline \multirow[t]{2}{*}{ Residual Heterogeneity (se) } & - & 0.012 & 0.022 \\
\hline & & $(0.003)$ & $(0.004)$ \\
\hline
\end{tabular}

Notes: ${ }^{b}$ these are used as a reference category. ${ }^{*}$ Significant at $\mathrm{p}<0.05 ; * *$ Significant at $\mathrm{p}<0.01 ; * * *$ Significant at $\mathrm{p}<0.001$. 'WLS weighted by inverse within-study variance of the effect sizes; ^ ${ }^{\wedge}$ WLS weighted by inverse within and between study variance multiplied by ISI impact factor.

\section{Discussion of Meta-regression Analysis}

Our analysis yields both counter-intuitive as well as confirming results. Column (3) and (4) of Table 2.3 show meta-regression results obtained by means of REML where effect sizes are weighted by the inverse of within-study variances, and the inverse of within-study variance 
plus between-study variance and ISI impact factor are used as a proxy for study quality. Inspecting these two columns and the last two rows of Table 2.2, the estimates and Rsquares do not appear to differ substantially. Therefore, adding the ISI impact factor offers a relatively little added value. Following Card and Krueger (2005), we also estimated the model with OLS, adding standard error in the regressor in order to check for publication bias. The results show no potential publication bias. In addition, the majority of moderator variables were insignificant. We cross-checked this result by calculating the fail-safe $\mathrm{N}$ of Rosenthal (1979) i.e. the numbers of 'missing' studies needed to nullify the result. The failsafe $\mathrm{N}$ seems very unlikely to exist $(\mathrm{N}>1000)$. Hence, we conclude that there is no threat for the publication bias in our meta study. We now continue to discuss the results reported in column (3).

\section{Feeling-Right Operationalization}

The regression analysis shows that effect sizes produced from process-based approach and outcome-based approach do not significantly differ ( $\mathrm{p}=0.598$ for outcome-based). While Aaker and Lee (2006) argue that both processes are distinct, the results from our empirical review do not corroborate this. For instance, these authors argue that it may be less likely that respondents experience the feeling right factor in outcome-based operationalizations. Future research should investigate whether alternative operationalizations lead to diverging experiences in RF study designs.

Types of Regulatory Focus

As shown in the table, the association between all types of priming and effect size is significant ( $p<0.05$ for ideal-ought, $p<0.001$ for identical tasks, $p<0.001$ for approach/avoidance and $\mathrm{p}<0.05$ for attribute priming). The coefficient estimates associated with these priming types have negative signs. Taking the RF trait as a reference category, this signifies that the effect of trait on the size of the effect is larger than those produced by all types of priming. While regulatory focus theory generally posits that the effects of trait and priming on behaviors are similar (see Higgins 2000), our results show that the strength of the impact of trait and priming are actually different. Moreover, this shows that the effect of each of priming type on the effect sizes are different in comparison to the reference category. These findings yield more insight on the role of RF as trait and as state in producing fit effects and present an interesting avenue for future regulatory fit research. Several approaches could be used. For instance, in an experimental design that induces state RF, the effect of RF trait can be controlled as a covariate employing structural equation modeling (Bagozzi and Yi 1989). Alternatively, the impact of RF trait on behavior, as measured by the use of a self-reported questionnaire, could be studied by incorporating the effect of state RF and its interaction with trait RF, in line with, for instance, the latent state-trait theoretical framework that employs repeated measures (as suggested by Steyer et al. 1999). 


\section{Research Domain}

Domain of the research was found to be significantly related to effect size. Having nondomain specific or laboratory studies as a reference category, the coefficient estimates associated with health $(\mathrm{p}<0.05)$, education $(\mathrm{p}<0.001)$, and various other domains $(\mathrm{p}<0.05)$ are significantly negative. This means that the effect size produced by health, educational and the rest category of domains is smaller than those of laboratory studies are more oriented towards cognitive processing and less towards a specific domain. This is surprising and in contrast to our expectations. However, this finding is in line with Idson et al. (2001) who suggest that a fit effect occurs when participants are requested to process information extra carefully. Following Avnet and Higgins (2006) we surmise that this may point to non-linear relationship between respondent motivational intensity and the size of the fit effect. We feel that aspects of linearity are an important issue for future research.

\section{The Use of Incentives}

The result show that effect sizes vary across incentive types. Monetary and course credit incentives were found to have a significant negative relationship with effect size $(\mathrm{p}<0.01$ and $\mathrm{p}<0.05$, respectively), while personal gifts do not $(\mathrm{p}=0.096)$. Using voluntary participation as a reference category, this signifies that that monetary and course credits produce lower effect sizes than voluntary participation. These results prompt an interesting agenda for future research, i.e., as to whether outcomes in RF research may be attributable to the type of incentives used. Moreover, if voluntary participation can be associated with a signal of low motivation, this important for the type of information processing and hence the effect on fit.

\section{Type of Participant}

The sign of the coefficient estimates associated with college students was found to be positive and statistically significant $(\mathrm{p}<0.01)$. This means that the effect sizes associated with college students were larger than those of high school students. Again, this is in contrast with our expectations which were based on the argument that college students can be expected to have a higher cognitive capacity and, therefore, would tend exhibit lower regulatory fit effect sizes. A recent position taken by Wang and Lee (2006) may account for our counter-intuitive findings. They argue that as a result of cognitive processing respondents are instigated to actively seek information that fits their regulatory focus. Again, this may be caused by the fact that the relationship between motivational intensity and the size of fit effect is essentially non-linear, and that more research is needed to determine the optimal point where the fit effect occurs (see Figure 2.1). 


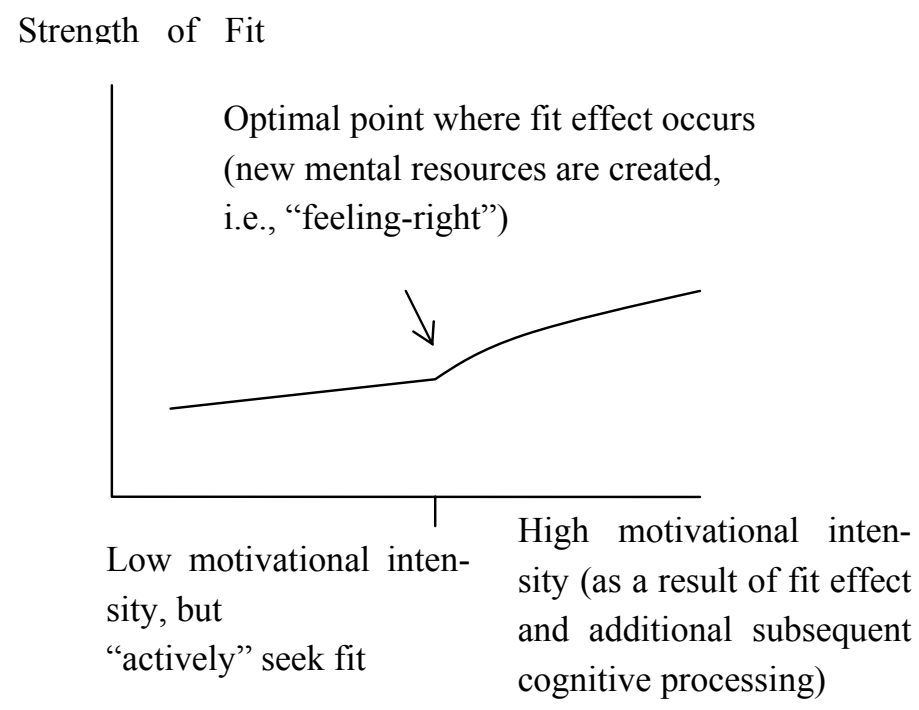

Figure 2.1: A Non-linear relationship with an optimal point effect

\section{Participant's Cultural background}

Finally, our results show that the coefficient estimate associated with a collective cultural background is not significant ( $\mathrm{p}=0.112$ ). Therefore, it seems that there is no association between this study characteristic and the size of the fit effect. One explanation for this is that the impact of culture on participants' regulatory focus may be confounded by the strength of regulatory focus manipulations. However, although individuals from individualistic cultures may have a tendency toward promotion focus and those from collectivist cultural backgrounds may lean toward prevention focus (Briley and Aaker 2006), the strength of their prevention or promotion may still vary considerably affecting the strength of RF manipulations and trait RF measure. Therefore, future research should attempt too unravel the interplay between cultural background and other major study variations.

\section{Conclusion and Limitations}

Overall, the strength of the fit effect varies between moderate and large. This empirically substantiates the fact that the fit effect does affect value creation in consumer decision making and as such plays an important role as a determinant of motivational intensity, attitudes and judgments, and behavioral change (Higgins 2005). Our results reveal that fit effects in studies examining dispositional traits produces stronger effects than those that induce $\mathrm{RF}$ (i.e., RF as state). Of the four types of RF inducement, attribute priming was found to produce the largest effects, followed by ideal-ought goal elicitation. This means that the efficiency of value enhancement may be higher when triggering consumer's regulatory focus 
with either one of these two types of inducements. We found that studies that do not provide incentives to stimulate participants' participation produce larger fit effects than those offering course credits or money. This is an interesting finding as many studies commonly provide incentives for participants in return for their participations with the aim of increasing their engagement in the studies. In addition, studies conducted in the context of personal health produce larger effect sizes than those conducted within the context of education, and that in studies that used college students stronger fit effects occur, relatively to those that use high school students. Despite the fact that collectivist cultures are commonly more associated with a prevention-focus and individualistic backgrounds are linked to a promotion focus (Lee and Aaker 2004), our study demonstrated that the fit effect appear to be robust across cultures.

Finally, following Aaker and Lee (2006), our meta-analysis study suggests that there seems to be a non-linear effect of motivational intensity on the strength of fit effect. We postulate that the effect may be characterized with an optimal point in which the fit effect occurs and above this point the effect of motivation on fit effect magnitude is strengthened (see Figure 1). However, future research is needed to shed light on this interesting relationship.

Our findings should be interpreted in the light of their limitations. First, we were only able to use a relatively small number of articles as only these met the imposed criteria for inclusion in the sample. Many articles did not contain enough information for calculation of the interaction effect size. Therefore, the generalizability of our results should be interpreted with some caution. Second, treating multiple effect sizes of the same studies as statistically independent may also create a certain degree of bias in the findings. As more effect sizes become available, future meta-analyses can be conducted by taking into account dependency among effect sizes. Third, in addition to the major study variables included in our analysis, other potential moderators may help to explain variation in effect sizes. For instance, we were unable to record the amount time participant spent in experiments, as such information is frequently not reported by researchers. The availability of such information may, for instance, help to better interpret the level of participants' involvement in the study. Overall, our review of the literature demonstrates the relevance of the regulatory fit effect. Future research seems no longer required to demonstrate its presence, but to further our understanding about when and why it occurs. 


\section{CHAPTER 3}

\section{Fitness by Fit: \\ A Regulatory Fit Perspective of Reward Preference in a Health Club Loyalty Program}

Two studies demonstrate that regulatory focus influences the choice of rewards in a loyalty program designed to stimulate repetitive behavior and member loyalty in a health club setting. In the first study, we find that the interaction between type of reward and the way the reward is communicated represents an important determinant of exercise intentions, perceived value, and program loyalty. In the second study, we reveal that consumer traits specific to the domain of loyalty programs mediate the influence of regulatory foci on goaldirected behavior or reward redemption decisions. These findings provide insights into the factors that drive consumers' reward preferences and choice of loyalty programs.

This chapter is largely based on: Daryanto, Ahmad, Ko de Ruyter, Martin Wetzels, and Paul G. Patterson. Fitness by Fit: A Regulatory Perspective of Reward Preference in a Health Club Loyalty Program. To be submitted for a $2^{\text {nd }}$ submission at the Journal of the Academy of Marketing Science. 


\section{Introduction}

Various service firms, including airlines, hotels, banks, and health clubs, attempt to formalize their relationships with customers through loyalty programs (LPs) (Uncles, Dowling, and Hammond 2003). As McKee (2007) reports, nearly 90\% of American consumers belong to a LP, averaging four programs per consumer. From the firm's perspective, LPs offer important advantages such as increased retention rates (Kivetz and Simonson 2002), opportunities for up- and cross-selling, and greater access to customer information that enables product or service customization (Uncles, Dowling, and Hammond 2003).

However, firms also face serious challenges related to LPs. First, programs with many customers are complex to administer (Dowling and Uncles 1997; Uncles, Dowling, and Hammond 2003). Second, because of their scale and increasing customer diversity, many LPs suffer decreased effectiveness, because one reward type cannot fit the preferences of all customer segments (Keh and Lee 2006; Kivetz and Simonson 2002). As a result, LPs may be creating liabilities instead of assets for firms (Nunes and Dreze 2006; Shugan 2005), especially if consumers save their earned points instead of using them to obtain program rewards. These concerns demand a deeper understanding of the design and positioning of LP reward structures to account for consumer preferences and help stimulate reward redemption (Kumar and Shah 2004).

Specifically, we use regulatory focus (RF) theory as a theoretical foundation to explain LP members' attitudes and behavior toward rewards, because it provides a powerful framework for explaining differences in individual goal pursuit motivations (Avnet and Higgins 2006). The theory posits two types of foci: a promotion focus, which attempts to achieve positive outcomes, and a prevention focus, aimed at avoiding negative outcomes (Higgins 1997). Typically, researchers use RF theory to explain how people process information framed in terms of either gains or losses and the manner of their goal pursuit, with the idea that the fit between regulatory focus and goal pursuit influences customer beliefs about the appropriateness of their (re)actions. Such regulatory fit also affects the perceived value of an object (e.g., specific reward) and can enhance motivational intensity (Avnet and Higgins 2006; Cesario, Grant, and Higgins 2004; Higgins 2002). We extend such theorizations to LPs and their rewards.

First, different types of rewards have divergent effects on consumer preferences for LPs (Keh and Lee 2006; Kivetz and Simonson 2002; Yi and Jeon 2003). By addressing the positioning (e.g., promotion vs. prevention) and communication (gain- or loss-framed) of rewards, RF theory predicts regulatory fit occurs when rewards emphasizing promotion (e.g., advancement and achievement, such as increased muscle strength) appear within gainframed information, and rewards emphasizing prevention (e.g., maintenance and security, such as constant exercise levels) are framed in terms of a potential loss (Lee and Aaker 2004; Wang and Lee 2006). This prediction relies on research that focuses almost exclu- 
sively on the effect of regulatory focus in relation to a single course of action (e.g., applying for a fellowship, evaluating the monetary worth of a mug) or in a single setting (e.g., studying student academic motivation, strategy for building long-lasting relationships). In contrast, this study theoretically supports and then empirically verifies the regulatory fit effect in relation to repetitive behavior and attitudes in the context of an LP.

Second, prior research frames regulatory foci as not only situational states but also individual traits (e.g., Lee and Aaker 2004), because in a LP context, redemption decisions and patronage behavior may depend on individual differences (i.e., promotion or prevention orientation). Recent research into the impact of traits (e.g., Chen, Gully, Whiteman, and Kilcullen 2000; Kanfer and Ackerman 2000) often distinguishes between distal and proximal personality characteristics, such that distal traits represent basic, general personality characteristics that are stable over time, whereas proximal traits reflect enduring dispositions to behave within certain situational contexts, which are malleable over time (cf. surface traits in marketing literature; Brown, Mowen, Donavan, and Licata 2002; Saxe and Weitz 1982 ). Proximal traits also differ from distal traits in that they associated more closely with the particular domain (Brown et al., 2002; Chen et al, 2000). Although conceptually, regulatory foci are considered distal traits, evidence about the possible mediating effects of consumer traits specific to the LP domain on their influence on goal-directed behaviors or redemption decisions is lacking. Therefore, we also examine whether proximal traits mediate the impact of regulatory foci on the choice of LP rewards.

Third, recent research reveals heterogeneity in consumer attitudes toward rewards, which results in diverging patterns of repatronage behavior (cf. Harris and Uncles 2007). Unobserved preferences provide only a weak basis for reward redemption predictions, because various factors influence the translation of attitudes (or lack thereof) into behavior (Fedorikhin and Cole 2004; Sirgy, Grzeskowiak, and Su 2005). These preferences also may not remain stable when program members actively process information about various types of rewards simultaneously. Thus, in addition to preference, we examine how regulatory foci influence actual consumer choice rewards. To address these issues, we examine the joint effects of reward type and message framing and then consider the influence of distal regulatory focus and domain-specific proximal traits on actual reward choice. Thus, this study offers a multimethod examination of the explanatory power of RF theory in relation to LP rewards.

As the context, we choose the LP of a health and fitness club, which enables us to investigate the fit effect in relation to repetitive behavior and attitudes (Study 1), in contrast with previous research that focuses on a single course of action (e.g., applying for a fellowship, getting a mammogram). Contemplating certain repetitive behaviors (e.g., exercising) may evoke either a prevention or a promotion mindset (Rothman, Bartels, Wlashin, and Salovey 2006), whose impact likely is stronger than the effects of thinking about one particular action. Furthermore, because exercising tends to be associated with prevention rather than promotion, it should prompt a prevention focus (cf. Worth 2005). We theorize that the 
level of involvement among people already engaged in a repetitive behavior should be higher than that among people who are just paying attention to a single course of action.

The U.S. Better Business Bureau recently revealed that the health club industry ranks within the top $1 \%$ in terms of the volume of customer complaints, that the majority of complaints relate to contractually enforced loyalty, and that churn rates run as high as $40 \%$ (McGovern and Moon 2007). Therefore, more and more health club service providers are experimenting with friendly ways to retain members, such as reward point-based LPs (Tock 2006). With the challenges that LPs present, it seems this industry has a particular need for greater understanding of the factors that drive consumer reward preference and choice, which may enable them to design effective LPs.

\section{Conceptual Framework and Hypotheses}

\section{Regulatory Focus Theory}

Regulatory focus (RF) theory proposes two distinct self-regulation strategies: promotionand prevention-focused (Higgins 1997). A promotion strategy emphasizes the pursuit of gains (e.g., achieving personal fitness) and the avoidance of nongains (e.g., avoiding being out of shape) and aspires to achieve and accomplish (so-called ideal goals). In contrast, a prevention strategy emphasizes the pursuits of nonlosses (e.g., maintaining good health) and the avoidance of losses (e.g., avoiding health problems), along with the fulfillment of duties, obligations, and security needs (so-called ought goals). Extant research demonstrates that promotion and prevention foci relate to distinct means for goal attainment. With a promotion focus, people tend to use an eagerness strategy. For example, health club members who want to gain physical strength attempt to increase the weight they lift. However, with a prevention focus, people tend to use a vigilance strategy and, for example, try to avoid missing training sessions. In addition to individual predispositions, regulatory focus can be conceptualized as a state or transient variable, such that it can be momentarily induced by a situation or task and by priming promotion versus prevention concerns (Crowe and Higgins 1997; Higgins 1997). According to Lee and Aaker (2004), researchers can frame a promotion focus by using phrases such as "diets rich in Vitamin $\mathrm{C}$ and iron lead to higher energy levels" in a health product context.

Advances in RF theory also attempt to explain the match between the type of goals and the strategy to achieve them, in the form of regulatory fit theory (RFT) (Higgins 2005). This theory posits that people engage more strongly or weakly in goal pursuit if the way to reach that goal sustains or disrupts their goal orientation. According to RFT, regulatory fit occurs when the manner of goal pursuit fits the person's individual regulatory orientation, such that a promotion-focused person uses an eagerness strategy and a prevention-focused person uses a vigilance strategy. In these situations, people feel right about what they are 
doing, which affects their perceptions of the value of the particular activity and results in stronger goal pursuit?

The principle of regulatory fit concept seems particularly relevant in the context of health club LPs, as the "feel right" factor should provide in-depth insights into the relationship among reward structures and revisit intentions, value perceptions, and choice behavior. This is because the experience of feeling right as a result of the match between reward types that functions as a prime for triggering regulatory focus and message framing can be transferred to the three constructs, as it intensifies aforementioned attitudes and behavior (Higgins 2005; Avnet and Higgins 2006). For example, if members believe that the reward has a high value; their experience of a fit effect (i.e., feeling right) should positively influence their revisit intentions.

We examine whether structuring LPs rewards to fit with promotion and prevention goals, and communicating them through eagerness (gains) and vigilance (loss) message frames, enhances the perceived value of the reward, promotes exercise participation, and enhances LP effectiveness. Therefore, we first consider the main effects of reward type and framing and their interaction on reward value perceptions and revisit intentions. Because regulatory fit creates value (i.e., value-from-fit, Higgins 2002), it affects consumer evaluations. In addition, we examine the effect on actual reward choices in a follow-up study.

\section{Main Effects of Reward}

According to RF theory (Higgins 1997), a prevention-focused health club member should prefer a prevention rather than a promotion reward, whereas the reverse is true for a promotion-focused member. Because exercising is predominantly associated with preventive behavior (Rothman and Salovey 1997), it may evoke more prevention focus concerns. Therefore, we argue that health club members should prefer prevention rewards. Recent research similarly demonstrates that some objects, situations, and behaviors relate more directly to either a prevention or promotion focus (Kay, Wheeler, Bargh, and Ross 2004). Therefore, the association between exercise and preventive behavior may activate prevention systems in memory, such that the term "exercise" functions like a stimulus prime that triggers a prevention focus, much like buying stock options triggers a promotion focus (Zhou and Pham 2004).Members of a health club thus should value prevention rewards more.

H1: Prevention rewards have a stronger effect on (a) perceived value and (b) revisit intentions than promotion rewards.

\section{Main Effects of Framing}

Considerable persuasion research examines the effect of message framing (i.e., gain vs. loss) on health-related behavioral intentions, such as breast self-exams (Banks, Salovey, and 
Greener 1995; Meyerowitz and Chaiken 1997), dieting (Spiegel, Grant-Pillow, and Higgins 2004), and exercise (Arora, Stoner, and Arora 2006; Jones, Sinclair, and Courneya 2003). These authors manipulate gain-framed messages by focusing on the benefits that result from performing the behavior described in either a gain or a nonloss situation, such as: "If you get a mammogram, you are likely to find out that your breasts are healthy" (gain) versus "If you get a mammogram, you decrease the risk of an undetected, potentially life-threatening tumor" (nonloss). For loss-framed messages, these studies focus on the risks or benefits that respondents would forgo if they did not perform the behavior associated with either a loss or a nongain situation, such as: "If you do not get a mammogram, you increase the risk of an undetected, potentially life-threatening tumor" (loss) versus "If you do not get a mammogram, you will not know whether your breasts are healthy." (nongain) (Salovey and Rothman 1997).

The framing effect varies depending on the context, such that loss-framed messages are more persuasive for advocating detection behaviors, such as breast-self exams, whereas gain-framed messages are more persuasive for prevention behaviors, such as exercising, because respondents perceive that performing the behavior will prevent unpleasant result (e.g., health problems) (e.g., Jones et al. 2003; Meyerowitz and Chaiken 1987; Rothman and Salovey 1997; ). Prospect theory offers a general theoretical explanation for these diverging findings (Kahneman and Tversky 1979; Tversky and Kahneman 1991) by arguing that when they face potential gains, people avoid risk (risk-averse), but when they confront a potential loss, they tend to take risks (risk-seeking). In a health-related context, detection behaviors may be associated more with risk than preventive behaviors, which implies a loss-framed message will be more persuasive than a gain-framed one. Because preventive behaviors constitute risk-averse actions, gain-framed messages should be more effective for advocating those behaviors (Rothman et al. 2006; Rothman and Salovey 1997). In our context, the risk pertains to the opportunity forgone in obtaining a reward (i.e., better fitness). Therefore, we hypothesize:

H2: Gain-framed messages have a stronger effect on revisit intentions than do loss-framed messages.

Although gain- and loss-framed messages both have persuasive impacts on behaviors, framing studies also reveal that message framing may influence the perceived value of the action and affect object priming (Rothman and Salovey 1997). Because an LP reward should be perceived as a gain, gain-framed messages may have a greater impact on the perceived value of the LP reward than do loss-framed messages.

H3: Gain-framed messages have a stronger impact on the value perceptions of LP rewards than do loss-framed messages. 


\section{Frame, Reward Type, and Interaction}

Although it reports some context-specific main and framing effects, recent research focuses primarily on their interaction in the form of RFT (Aaker and Lee 2006; Avnet and Higgins, 2006), because regulatory fit explains and predicts subjects' attitudes and behaviors well (Higgins 2005; Spiegel et al. 2004). Several studies even report that this interaction provides the only robust predictor of attitudes and behavior (Lee and Aaker 2004; Cesario et al. 2004, Freitas and Higgins 2002). Lee and Aaker (2004), treating RF as a state variable, also demonstrate that a fit effect occurs when (1) subjects process information that addresses their promotion concerns and then view gain-framed information or (2) they confront prevention concerns and loss-framed information.

In the LP context, health club members typically evaluate promotion (e.g. fitness check up) and prevention (e.g. dietary advice) rewards; therefore, consistent with RFT, people evaluating promotion rewards should perceive the higher value of the reward and indicate more intentions to exercise when they receive a gain-framed rather than loss-framed message. In contrast, members who evaluate prevention rewards perceive such value and intend to exercise when they view loss-framed messages.

H4: The perceived value of a reward is greater for members who evaluate promotion rewards with gain-framed messages, whereas members evaluating prevention rewards with loss-framed messages perceive greater value of the reward.

H5: Members evaluating promotion rewards with gain-framed messages experience a higher intention to exercise, whereas for members evaluating prevention reward with loss-framed messages indicates a higher intention to exercise.

\section{Value Perceptions, Revisit Intentions, and Loyalty}

Rewards offered in a health club context typically have direct benefits in support of exercise motives and health improvement (e.g., dietary advice, fitness check-up). Because of their relevance to the member's exercise goals, these rewards should appear valuable, which may lead to increased motivation to exercise. Similarly, but in a different context, perceptions of postpurchase value correlate strongly with repurchase intentions (Petrick and Backman 2002).

H7: Value perceptions relate positively to revisit intentions.

In turn, revisit intentions should have a positive relationship with LP loyalty, because points accumulated through regular exercise likely represent a sequential process of involvement in the activity, during which members develop a psychological commitment to the LP (Iwasaki 
and Havitz 1998). Thus, members committed to exercise to gain points should be more loyal to the LP than those who are not.

H8: Member's revisit intentions relate positively to loyalty program commitment.

Previous LP research reveals a positive relationship between reward perceptions and LP attractiveness (Kivetz and Simonson 2002, 2003; Yi and Jeon 2003). Rothschild and Gaidis (1981) contend that if customers have an intrinsic interest in the product, LPs can induce loyalty to the program rather than to the product. Our research context seems a likely candidate for this contention, because members who derive value perceptions from the reward (which supports their exercise motives) attribute their value perceptions to the LP. In other words, members may translate their value perceptions into program loyalty, such that members with high rather than low value perceptions become more loyal to the LP.

H9: Value perceptions relate positively to loyalty program commitment.

We summarize our hypotheses in the conceptual framework in Figure 3.1.

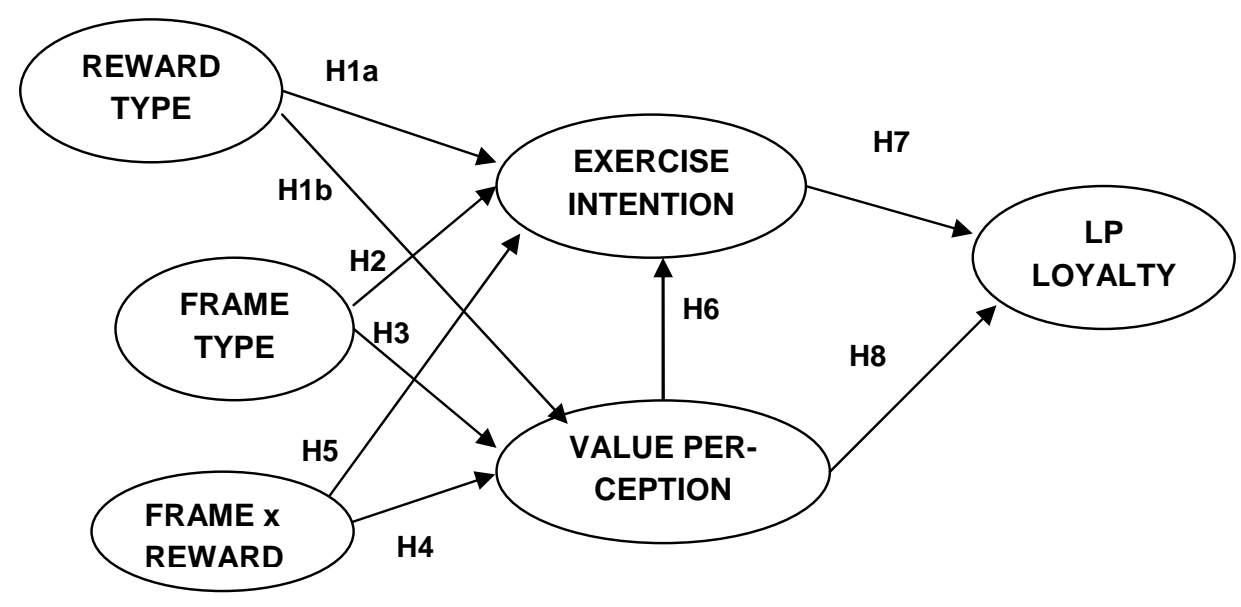

Figure 3.1: Conceptual Framework

\section{STUDY 1}

\section{Research Setting}

Our study context involves a LP administered by a health club with a wide range of health and fitness facilities that offers specific health programs for adult (e.g., fitness and exercise services) and youth (e.g., swim school) members. As evidence of its commitment to its customer relationship management program, it is one of few health clubs that uses a busi- 
ness-to-consumer portal on an online community forum to provide more services to its members.

To test our hypotheses, we design a $2 \times 2$ between-subjects experiment in which we manipulate the type of reward (dietary advice vs. fitness check-up) and type of frame (gain versus loss). We randomly assign the respondents to one of the four scenarios and collect data from a questionnaire. The questionnaire provides respondents with general instructions, scenario descriptions, and a list of items that measure regulatory focus, exercise motives, exercise intentions, value perceptions, and LP loyalty. We also include items to assess demographic details, including age, gender, occupation, and frequency and type of exercise.

\section{Procedure and Scenarios}

The scenario descriptions ask respondents (health club members) to imagine they had been working out at their health club for some time and were told they could earn a loyalty point every time they visited the gym facilities. If they reached 100 points, which they could do by exercising regularly at least twice a week, they could spend the points on either dietary advice or a fitness check-up. Furthermore, we emphasize the benefits of both rewards using text adapted from the research scenario employed by Jones et al. (2003), namely, emphasizing prevention and avoidance for the dietary advice reward (e.g., avoiding decreased metabolism) and improvement and accomplishment for the fitness check-up reward (e.g., improving physical exercise). After they read the description, respondents viewed messages framed as either losses or gains, with the aim of persuading them to accumulate points to obtain the reward. We include a sample scenario to illustrate the manipulation in the Appendix A.

\section{Pretest}

We administered a pretest to verify the ecological validity of the scenarios and the reward manipulation. On the basis of these results, we made several textual adaptations to enhance the effect of the reward priming manipulations. The final versions of the scenarios mention three prevention or promotion benefits for each type of reward. The pretest also reveals that respondent members have no difficulty imagining themselves in the scenario and that the manipulation of reward type works as intended.

\section{Construct Development}

The model contains three constructs: value perceptions, exercise intentions, and LP loyalty. All constructs use seven-point Likert scales anchored at strongly disagree (1) and strongly 
agree (7). The scales include multiple items, the wording for which we adapt to the characteristics of the research setting and scenario. The three-item loyalty measure is based on previous research by Yi and Jeon (2003) and McMullan and Gilmore (2003). To measure value perceptions, we use three items adapted from the scale developed by Yi and Jeon (2003). Our three-item measure for exercise intentions also is based on previous research by Jones et al. (2003). We list the measurement items in Table 3.1.

Table 3.1: Measurement Instruments

Items

Standardized

Loading

\section{Value Perceptions}

I think positively about the value of my health club's loyalty-program

0.580

I believe that my health club's loyalty-program is valuable

0.562

My overall evaluation of the loyalty program is positive

0.592

The proposed rewards have a higher cash value

0.841

it is highly likely to get the proposed rewards

0.823

The proposed rewards are what I have wanted

0.659

\section{Exercise Intentions}

In the next two weeks, my goal is to exercise in the health club

I intend to exercise at least every other day over the next two weeks in the health club

Over the next two weeks, I intend to exercise at least two times per week in health club

\section{Loyalty Program Commitment}

I appreciate the loyalty program.

I have a strong preference for this particular loyalty program because the program is appealing.

I would recommend this loyalty program to others.

We also include items as manipulation checks for reward type. Different items apply to each type of reward, rated on seven-point Likert scales that range from strongly disagree (1) to strongly agree (7). For the fitness check-up scenarios, we include: "I believe having a fitness check-up can improve my physical exercise" and "I believe having a fitness check-up can help my goal of improving my physical exercise." For the dietary advice reward scenarios, we use: "I see the reward as a way to maintain my exercise level" and "I see the reward as a way to maintain a good health condition." In the manipulation checks of these rewards, we use a test value of 4 , because a value greater than the scale midpoint implies the reward represents promotion or prevention rewards. 


\section{Data Collection}

We collect data from questionnaires sent to health club members online. After screening the responses for incompleteness and excluding suspected outliers, we obtain a usable sample of 131 questionnaires. Fifty-eight percent of the respondents are female, and $88 \%$ of them exercise or use the pool at the gym on a regular basis. Of the respondents, $33 \%$ and $32 \%$ use the pool and the fitness center, respectively, as their primary form of exercise at the gym. Finally, $25 \%$ of respondents work out or use the pool at the gym three times per week.

\section{Data Analysis}

We use partial least squares (PLS) path modeling to test the hypotheses, specifically, PLSGraph software version 3.0 (Chin 2001), which we employ to obtain estimates for the parameters in the measurement and structural models. Compared with traditional covariancebased analysis or LISREL, the PLS method has several advantages, including the ability to handle nonmultivariate normal data, multicollinearity among independent variables, small samples, and formative indicators (Chin 2001; Tenenhaus, Vinzi, Chateline, and Lauro 2005). In addition, PLS is suitable for modeling factorial designs; unlike traditional experimental design techniques (e.g., (M)AN(C)OVA), PLS can control for measurement error, incorporate covariates in the model, and offer greater modeling flexibility (Mackenzie 2001).

To model our experimental data with structural equation modeling, we specify single indicators for the manipulated factors, namely, types of reward and types of frame, as well as the interaction effect. We code these manipulations with effect coding, such that fitness check-up is coded 1 and dietary advice as -1 , and the gain frame is coded 1 and the loss frame as -1 . Effect coding is more appropriate for categorical predictors in a multiple regression analysis of factorial designs than dummy coding $(0,1)$, because it produces tests of the main effects and interactions for any models involving interaction terms (O'Grady and Medoff 1988; Pedhazur 1997).

We specify the interaction between payoff and reward as the product term of the two effect-coded variables (reward $\times$ frame). The dependent variables - exercise intention, value perception, and loyalty - represent reflective indicators. We next examine the reliability, convergent validity, and discriminant validity of these indicators and provide the PLS parameter estimates for the measurement model in Table 3.2. 
Table 3.2: Composite Reliability and Correlation among Construct Scores

\begin{tabular}{lllllllll}
\hline Construct & CR & AVE & $\mathbf{1 .}$ & $\mathbf{2 .}$ & $\mathbf{3 .}$ & $\mathbf{4 .}$ & $\mathbf{5 .}$ & $\mathbf{6 .}$ \\
\hline 1. FRA & na & na & $\mathbf{n a}$ & & & & & \\
2. REW & na & na & -0.070 & $\mathbf{n a}$ & & & & \\
3. FRA $\times$ REW & na & na & 0.553 & 0.023 & $\mathbf{n a}$ & & & \\
4. VALUE & 0.838 & 0.571 & 0.087 & -0.192 & 0.010 & $\mathbf{0 . 6 8 6}$ & & \\
5. EXE & 0.867 & 0.684 & 0.010 & -0.053 & 0.027 & 0.227 & $\mathbf{0 . 8 2 7}$ & \\
6. LOY & 0.901 & 0.771 & 0.117 & 0.066 & 0.102 & 0.462 & 0.308 & $\mathbf{0 . 8 7 8}$ \\
\hline Notes: Average variance extracted square root values on the diagonal. FRA = frame type, \\
REW = reward type, VALUE = value perception, EXE = exercise intention, LOY = program \\
loyalty, CR = composite reliability, and AVE = average variance extracted. Dummy coding \\
is used for main effects.
\end{tabular}

To assess the internal consistency of a given block of indicators, we employ composite reliability (CR) (Fornell and Larcker, 1981) and average variance extracted (AVE). As we show in Table 3.2, the CR scores for value perceptions, exercise intentions, and loyalty are $0.838,0.867$, and 0.901 , respectively, which exceed the cut-off value of 0.7 proposed by Nunnally and Bernstein (1994). The AVE for value, exercise intentions, and loyalty are $0.571,0.684$, and 0.771 , respectively, which again exceed the cut-off value of 0.5 suggested by Fornell and Larcker (1981).

To assess the discriminant validity of each construct, we use three methods. First, we note that a construct should share more variance with its measures than with other constructs (Chin 1998; Hulland 1999), so the square root of the AVE should exceed the intercorrelations of the construct with other constructs in the models. As we display in Table 3.2, the square root of the AVE for all constructs is greater than all corresponding correlations (Fornell and Larcker 1981). Second, cross-loadings with constructs other than the hypothesized construct are not substantial in magnitude $(<0.3$; Chin 1998; Hulland 1999). Third, the correlations of the residual terms across blocks do not exceed |0.2| (Falk and Miller 1992; Fornell and Bookstein 1982). Therefore, all constructs exhibit satisfactory discriminant validity.

We assess the convergent validity of the constructs by inspecting their factor loadings. In Table 2.1, we demonstrate that the factor loadings for value perceptions, exercise intentions, and loyalty are all greater than 0.50 , which demonstrates acceptable convergent validity.

Finally, we check for common method variance in our data, which may arise if the self-reported measures come from the same person in the same measurement context using similar item characteristics (Podsakoff, Mackenzie, Lee, and Podsakoff 2003). We use Harman's single-factor test and conduct an exploratory factor analysis to extract the unrotated factor solution by including all items. Common method variance exists if a single factor emerges that accounts for the majority of the covariance. However, our results indicate that no single factor with an eigenvalue greater than 1 accounts for more than $34 \%$ of the variance. Therefore, common method variance does not appear to be a problem for our data. 


\section{Results}

\section{Manipulation Check}

Our manipulation checks for both rewards (dietary advice: $\mathrm{t}(62)=6.99, p<0.001$; fitness check-up: $\mathrm{t}(67)=8.47, p<0.001)$ are significantly different from the scale midpoint. Therefore, the manipulations resulting from the scenarios produce the intended effect (Perdue and Summers 1986).

\section{Hypotheses Testing}

We display the PLS estimates of the structural model in Table 3.3. The path coefficient estimates result from a nonparametric bootstrapping procedure with 500 resamples (Chin 1998). As the results show, only the main effect of rewards on value perceptions is significant, in support of $\mathrm{H} 1 \mathrm{~b}(\beta=-0.197, \mathrm{t}=3.418)$. That is, prevention rewards seem to have a stronger effect than promotion rewards on reward value perceptions. The effects of reward type on exercise intention (H1a) and of frame type on exercise intention (H2) and value perceptions (H3) are not significant, so we must reject these hypotheses. We also fail to find a significant interaction effect between reward type and frame type on exercise intention, so we find no support for H5. However, we find a significant positive interaction effect between reward type and frame type on value perceptions $(\beta=0.125, \mathrm{t}=2.775)$, in support of H4 (see Figure 3.2).

Table 3.3: Results for Structural Model

\begin{tabular}{llcrl}
\hline Hypothesis & Relationship & Estimate & t-values & Result \\
\hline H1a & REW $\rightarrow$ VAL & -0.197 & 3.418 & supported \\
H1b & REW $\rightarrow$ EXE & -0.017 & 0.831 & not supported \\
H2 & FRA $\rightarrow$ EXE & -0.011 & 0.767 & not supported \\
H3 & FRA $\rightarrow$ VAL & 0.068 & 0.514 & not supported \\
H4 & REW $*$ FRA $\rightarrow$ VAL & 0.125 & 2.775 & Supported \\
H5 & REW $*$ FRA $\rightarrow$ EXE & 0.069 & 0.946 & not supported \\
H6 & VAL $\rightarrow$ EXE & 0.218 & 2.499 & Supported \\
H7 & EXE $\rightarrow$ LOY & 0.203 & 2.421 & Supported \\
H8 & VAL $\rightarrow$ LOY & 0.438 & 4.138 & Supported \\
\hline
\end{tabular}

Fit measure $\mathrm{R}^{2}: \mathrm{VAL}=0.058, \mathrm{EXE}=0.055, \mathrm{LOY}=0.257$

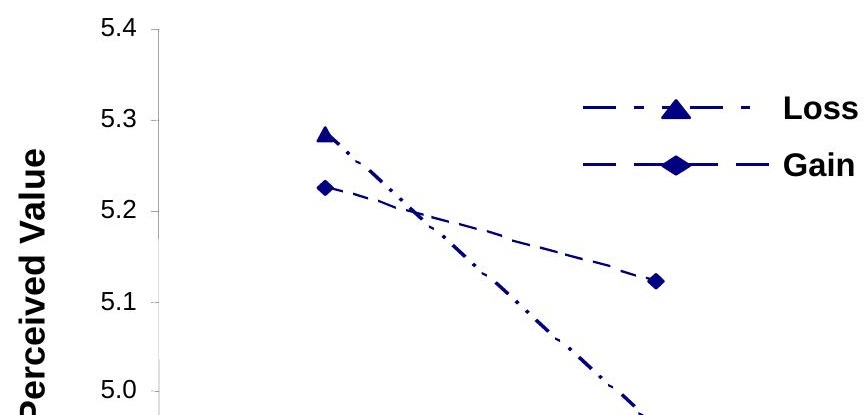


Figure 3.2: Interaction between Reward and Frame on Reward Value Perception

Considering this significant interaction effect, we conduct two simple effect tests to shed light on the nature of the interaction (Keppel and Wickens 2004; Pedhazur 1997) in which we examine the differences between gain and loss frames separately for promotion and prevention rewards. Specifically, we conduct (1) a test of the mean differences of gainversus loss-framed messages for the fitness check-up reward and (2) a test of the mean differences of gain- versus loss-framed messages for the dietary advice reward. Thus, we split the data into two groups according to the reward category. We again use effect coding, such that the gain frame is 1 and the loss frame is -1 . Next, we regress value perceptions on the frame type underlying each type of reward. We use PLS-Graph with nonparametric bootstrapping of 500 replications to obtain the parameter estimates for the relationship between frame type and value.

For the fitness check-up reward $(\mathrm{n}=68)$, we obtain a positive and significant relationship between frame type and value $(\beta=0.230, \mathrm{t}=3.744)$, such that with a promotion reward, value perceptions are greater in the gain frame than in the loss frame. For the dietary advice reward $(n=63)$, we obtain a negative, marginally significant relationship between frame type and value $(\beta=-0.123, \mathrm{t}=1.864)$, such that with a prevention reward, value perceptions are greater for the loss than for the gain frame. Altogether, these results support H4.

With respect to the interrelationship among value perceptions, exercise intentions, and loyalty, we find a significant, positive relationship between value perceptions and exercise intentions $(\beta=0.218, \mathrm{t}=2.499)$, in support of $\mathrm{H} 6$; between exercise intentions and loyalty $(\beta=0.203, \mathrm{t}=2.412)$, in support of $\mathrm{H} 7$; and between value perceptions and loyalty $(\beta=0.438, \mathrm{t}=4.138)$, in support of $\mathrm{H} 8$.

\section{Effect of Reward Type on Actual Reward Choice}

In the preceding sections, we assume that evaluations of rewards can induce a regulatory focus, such that evaluations of prevention rewards trigger a prevention focus and evaluations of promotion rewards induce a promotion focus. In the second study, we examine the 
influence of trait RF and exercise motives on actual reward choices rather than intentions, to determine (1) whether consumers are active recipients of information who seek to create fit when they evaluate two different types of rewards simultaneously (Wang and Lee 2006) and (2) whether the results are valid for both intentions (preferences) and actual behavior (choice). People sometimes fail to make a consistent choice based on their preferences, due to other factors such as social approval (Sirgy et al. 2005) or mood (Fedorikhin and Cole 2004).

As we noted previously, research into the impact of traits on motivation (Kanfer and Ackerman, 2000) distinguishes between distal personality characteristics, which are more general and have a more indirect impact on attitudes, and proximal personality constructs, which mediate the impact of distal constructs on behavior (Chen et al. 2000) because they are specific to situations and malleable over time.

In contrast, we propose that the proximal trait of exercise motivation (Markland and Ingledew 1997) mediates the impact of a distal regulatory focus on choice. Specifically, the influence of a promotion focus on choice should be mediated by achievement fexercise motives, whereas the influence of a prevention focus is mediated by avoidance motives. Achievement exercise motives constitute a desire to achieve better health, which reflects a promotion focus, whereas avoidance implies a desire to avoid negative end states, such as poor health, and thus reflects a prevention focus.

H9. A health club member's (a) promotion focus relates positively to achievement motives and (b) prevention focus relates positively to avoidance motives.

H10. A health club member's (a) achievement motives relate positively to the choice of promotion rewards and (b) avoidance motives relate positively to the choice of prevention rewards.

We summarize these latter hypotheses in the conceptual framework depicted by Figure 3.3.

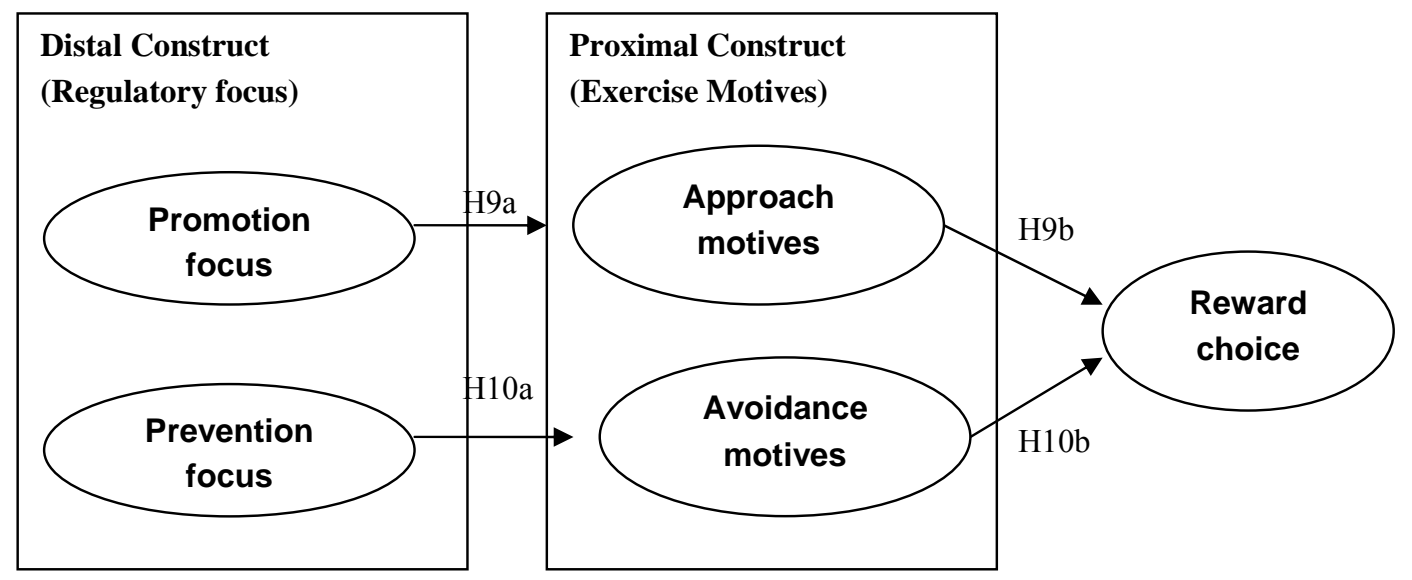

Figure 3.3: Impact of Distal and Proximal Constructs on Actual Reward Choice 


\section{STUDY 2}

The study context is the same as that in Study 1, and we again collect data through a questionnaire that contains general instructions, scenario descriptions, and items to measure regulatory focus, exercise motives, and reward choice (seven-point Likert scales anchored at strongly disagree [1] and strongly agree [7]). We use multiple items for which we adapt the wording to the characteristics of the research setting and scenario. The binary reward choice variable indicates the respondent's choice (dietary advice or fitness check-up). To measure trait regulatory focus, we adapt a scale developed by Lockwood, Jordan, and Kunda (2002) to tap members' chronic regulatory focus. The scale consists of two subscales designed to measure chronic promotion and prevention focus strength. For exercise motives, we borrow items from the exercise motivation inventory (EMI-2) developed by Markland and Ingledew (1997). We categorize these items into achievement and avoidance motives on the basis of ratings by six independent judges (marketing doctoral students). Using the definitions of the achievement (e.g., something that people want to get as a result of exercising) and avoidance (e.g., something that people want to avoid by exercising) exercise goals derived from goal orientation literature (Worth et al. 2005), the judges reviewed the EMI-2 items, then classified each into one of four categories: achievement, avoidance, both, or neither. To assess the proportion of agreement among judges, we use the proportional reduction in loss (PRL) reliability measure (Rust and Cooil 1994), which indicates five items that tap an avoidance exercise motive and three items that refer to an achievement exercise motive (interjudge agreement $=0.6, \mathrm{PRL}=0.85$ ).

The survey in Study 2 results in usable data from 222 respondents, $50 \%$ of whom are younger than 26 years of age. On the basis of weekly exercise frequency, we classify $6 \%$ of these respondents as light users (1-2 times/week) and $6 \%$ as heavy users ( $>5$ times/week). We also discover that the proportions of promotion- and prevention-focused respondents in the sample are approximately equal (52\% promotion-focused). In addition, among respondents who chose the fitness check-up, the percentage of promotion-focused users is greater than prevention-focused (66\% vs. $56 \%$ ), whereas dietary advice is preferred most among prevention-focused respondents ( $38 \%$ vs. $34 \%$ ).

\section{Results}

We use covariance-based modeling, with Mplus software version 4.0 with a WLSMV (weighted least squares mean and variance adjusted) estimator, to test our hypotheses. This software provides advanced capability for handling models with binary choice variables (Muthen 1984).

We test two models, one of which is nested in the other. We also specify direct and indirect effects of distal traits on choice (partial mediation) in one model but no direct effects in the other model (complete mediation). In the partial mediation model, both direct 
paths of promotion $\rightarrow$ choice $(\mathrm{t}=0.109)$ and prevention $\rightarrow$ choice $(\mathrm{t}=-1.158)$ are insignificant; therefore, a complete mediation effect exists. We continue by discussing only the results of the complete mediation model.

Table 3.4: Measurement Instruments

\begin{tabular}{ll}
\hline Items & $\begin{array}{l}\text { Standardized } \\
\text { loading }\end{array}$ \\
\hline Promotion focus & \\
I am frequently concerned with how I can achieve my hopes and aspirations. & 0.636 \\
I often think about the person I would ideally like to be in the future. & 0.687 \\
I typically focus on the success that I hope to achieve in the future & 0.700 \\
My major goal in exercising right now is to achieve my exercising goals. & 0.691 \\
& \\
Prevention focus & 0.821 \\
In general, I am focused on preventing negative events in my life. & 0.751 \\
I am anxious that I will fall short of my responsibilities and obligations.. & 0.575 \\
I often think about the person I am afraid I might become in the future. & 0.585 \\
I often worry that I fail to accomplish my goals in life. & \\
& \\
Approach exercise motives & 0.831 \\
To develop personal skills & 0.879 \\
To increase my endurance & 0.796 \\
To get stronger & 0.917 \\
To give me personal challenges to face & \\
Avoidance exercise motives & \\
To prevent health problems & 0.830 \\
To help manage my stress & 0.878 \\
Because exercise helps me to burn calories & 0.844 \\
\hline
\end{tabular}

In table 3.4, we list the standardized factor loadings for the measurement model. Because the factor loadings for all constructs are greater than 0.50 , acceptable convergent validity exists. In Table 3.5, we provide the correlations among construct scores, CR, and AVE, as well as the square root of the AVE. All measures exceed the cut-off values of $0.70(\mathrm{CR})$ and 0.50 (AVE). Moreover, the square root of the AVE exceeds the intercorrelations of the construct with other construct; therefore, all constructs exhibit satisfactory discriminant validity.

Table 3.5: Composite Reliability and Correlation among Construct Scores

\begin{tabular}{lccccccc}
\hline Construct & CR & AVE & $\mathbf{1 .}$ & 2. & 3. & 4. & 5. \\
\hline & & & & & & & \\
1. PRO & 0.748 & 0.526 & $\mathbf{0 . 6 5 3}$ & & & & \\
2. PRE & 0.733 & 0.523 & 0.425 & $\mathbf{0 . 6 5 0}$ & & & \\
3. APH & 0.914 & 0.728 & 0.274 & 0.116 & $\mathbf{0 . 8 5 3}$ & & \\
4. AVO & 0.877 & 0.705 & 0.173 & 0.407 & -0.091 & $\mathbf{0 . 8 3 9}$ & \\
5. CH & na & na & -0.051 & -0.128 & 0.043 & -0.319 & na. \\
\hline Notes: Average variance extracted square root values on the diagonal. PRO =
\end{tabular}


promotion focus, $\mathrm{PRE}=$ prevention focus, $\mathrm{APH}=$ achievement motive, $\mathrm{AVO}=$ avoidance motive, $\mathrm{CH}=$ binary choice, $\mathrm{CR}=$ composite reliability, and $\mathrm{AVE}=$ average variance extracted.

Table 3.6: Results for Structural Model

\begin{tabular}{llcclll}
\hline & & & \multicolumn{3}{c}{ Bootstrap } \\
Hypothesis & Relationship & Coefficient & t-values & Coefficient & 95\% CI* & Result \\
\hline $\mathrm{H} 10 \mathrm{a}$ & PRO $\rightarrow$ APH & 0.341 & 2.936 & 0.305 & $0.039,0.571$ & supported \\
H10b & PRE $\rightarrow$ AVO & 0.671 & 3.264 & 0.605 & $0.301,1.181$ & supported \\
H11a & APH $\rightarrow$ CH & 0.01 & 0.126 & 0.042 & $-0.126,0.187$ & not supported \\
H11b & AVO $\rightarrow$ CH & -0.203 & 2.901 & -0.205 & $-0.384,-0.058$ & supported \\
Indirect effect & PRO $\rightarrow$ APH $\rightarrow$ CH & & & 0.013 & $-0.042,0.08$ & not supported \\
& PRE $\rightarrow$ AVO $\rightarrow$ CH & & -0.124 & $-0.290,-0.028$ & supported \\
& Model fit index & & & & \\
& $\chi 2$ (df), df=28 & 33.384 & & & & \\
& p-value & 0.225 & & & & \\
CFI & 0.965 & & & & \\
TLI & 0.972 & & & & \\
& RMSEA & 0.034 & & & & \\
WRMR & 0.692 & & & & \\
\end{tabular}

$\mathrm{R}^{2}: \mathrm{AVO}=0.165 ; \mathrm{APH}=0.075 ; \mathrm{CH}=0.102 . *$ Bias-corrected confidence interval.

Finally, in Table 3.6, we provide the parameter estimates of the structural model and statistics commonly reported by Mplus as a means to assess model fit. The model attains very good fit $(\chi 2(28)=33.384, p=0.225$, root means square error of approximation [RMSEA] $=$ 0.034 , confirmatory fit index $[\mathrm{CFI}]=0.965$, Tucker-Lewis index $[\mathrm{TLI}]=0.972$, Weighted Root Mean Square Residual [WRMR] = 0.692), according to usual conventions (Kline 1998).

The results confirm most of our hypotheses. We find a significant, positive relationship between promotion focus and achievement motives $(\beta=0.341, t=2.936)$, in support of H9a. We also find a significant, positive relationship between a prevention focus and avoidance motives $(\beta=0.671, t=3.264)$, in support of H9b. However, we fail to find a significant relationship between achievement and the choice of the fitness-check reward $(\mathrm{t}=$ 0.126), so we must reject H10a. Finally, we find a significant, negative relationship between avoidance and the choice of the dietary advice reward $(\beta=-0.203, t=2.901)$. That is, the choice of a prevention reward is influenced by avoidance motives.

Following Mallinckrodt, Abraham, Wei, and Russell (2006), MacKinnon, Lockwood, and Williams (2004), and Shrout and Bolger (2002), we conduct a bootstrap test of indirect effects using Mplus software version 4.0, a method useful for testing the statistical significance of the mediation effect, because it has greater statistical power to detect true mediation effects relative to other methods (e.g., normal theory approach, Baron and Kenny 1986), especially with small sample or effect sizes. It also provides greater precision when 
calculating confidence intervals, regardless of the sample or effect sizes, and considers the nonnormality of the distribution of the parameter estimates.

We use original data from 222 cases to generate 1000 bootstrap samples and then calculate the $95 \%$ bias-corrected confidence interval (MacKinnon et al. 2004). This confidence interval corrects for bias due to the nonnormality of the distribution of sampling estimates. The bias-corrected 95\% confidence interval appears in the last three columns of Table 3.6. The interval for the indirect path of $\mathrm{PRO} \rightarrow \mathrm{APH} \rightarrow \mathrm{CH}$ is $(-0.042,0.08)$, which includes 0 ; that for $\mathrm{PRE} \rightarrow \mathrm{AVO} \rightarrow \mathrm{CH}$ is $(-0.290,-0.028)$, which does not include 0 . Therefore, the indirect effect for prevention foci is statistically significant at the $5 \%$ level, whereas for the promotion foci, it is not significant at the 5\% level. Overall, our hypothesis that the influence of (distal) regulatory foci on choice is mediated by proximal traits of exercise motives is marginally supported.

\section{Discussion}

With this study, we attempt to study members' reactions to LP rewards with RFT as our theoretical basis. Specifically, we examine the interaction effect between the manner in which the reward is positioned and the way it gets communicated (gain or loss) (Study 1). This reward positioning constitutes specific regulatory states. Moreover, we examine the impact of regulatory and domain-specific traits on consumer reward choices (Study 2) while considering preference stability when program members actively process information about various types of rewards simultaneously.

In Study 1, the results show that reward type and message framing do not have a significant impact on exercise intentions, though their interaction has a positive impact on reward value perceptions. Therefore, value perceptions appear to mediate the joint effect of reward type and frame type on exercise intention, in line with RFT (Aaker and Lee 2006; Lee and Aaker 2004). In addition to this joint effect, we find that prevention rewards seem to have a stronger effect on value perceptions than do promotion rewards. This main effect may occur because people tend to associate exercise with prevention rather than promotion (cf. Worth et al. 2005). Therefore, members likely are attracted to prevention- rather than promotiontype rewards. In addition, we find a significant influence of value perceptions and exercise intentions on LP loyalty, which corroborates prior research in LP settings (Yi and Jeon 2003).

In Study 2, we further demonstrate that reward choice is influenced by regulatory focus and domain-specific proximal traits. A complete mediation effect means regulatory foci affect the choice of reward. Specifically, the choice of a prevention reward likely gets facilitated when people hold a prevention focus and avoidance motives. Although we find that a promotion focus relates positively to achievement motives, we do not find any significant indirect effect of promotion focus. Perhaps the domain of our study is more associated with prevention than promotion actions, which would corroborate our finding of a main effect of 
the prevention reward in Study 1. Furthermore, the two studies provide convergent evidence that the influence of regulatory focus on member behavior for LPs may be context dependent. These findings further imply an influence of prevention focus on customer responses in a prevention-type domain. Unlike Study 1, Study 2 involves two rewards, presented simultaneously to participants. Therefore, participants were not merely passive recipients of information but instead actively selected information that fit their regulatory orientation (Wang and Lee 2006). In turn, persons with a stronger promotion focus may indicate a higher rating for achievement motives measures, whereas those with a stronger prevention focus likely rate avoidance motive measures higher. Our findings seem to reflect these claims. Overall, we conclude that members' reactions to LP rewards seem influenced by their regulatory focus and the domain-specific construct of exercise motives. In this sense, our results indicate the crucial importance of fit between reward positioning and communication to LP members.

\section{Limitations and Future Research Directions}

Any study must be evaluated in the light of its limitations, and this study is no exception. First, according to RFT (Cesario et al. 2004; Higgins 1997), gain frames refer to the attainment of positive outcomes (gain) or the failure to attain negative outcomes (nonloss), whereas loss frames focus on attaining negative outcomes (loss) or not attaining positive outcomes (nongain). In our experimental setting, we manipulate gain and loss frames in terms of the benefits of exercise as a gain and those benefits not being attained for the loss frame. We do not include a nonloss situation for the gain frame (e.g., "if you exercise, you will not fail to get a sufficient amount of credit points") or a nongain situation for the loss frame (e.g., "if you don't exercise, you will not earn a sufficient amount of credit points"). The effect of framing on persuasion is much greater for gain-loss situations than nonlossnongain situations (Cesario et al. 2004), and regulatory focus fit effects on persuasion occur for these cells (Lee and Aaker 2004). However, further research might replicate our findings by taking into account the four frames (gain, nongain, loss, nonloss) to obtain conclusive findings about LPs in the health-related communication domain.

Second, our experimental design includes different types of rewards featuring promotion and prevention concerns (i.e., promotion fitness check-up, prevention dietary advice). An alternative approach might present one type of reward and frame it with either promotion or prevention concerns (i.e., attribute framing; Levin, Schneider, and Gaeth 1988). Positive frames (e.g., gains, success) are more effective in the context of attribute framing than are negative frames (Krishnamurthy, Carter, and Blair 2001). Therefore, additional research should investigate how this effect interacts with regulatory focus in the context of LPs in the health domain.

Third, our research context has high self-relevance for respondents, so their intrinsic motivation to process the information should be relatively high. Research by Krishnamurthy 
et al. (2001) demonstrates only partial support for the effect of framing in such highinvolvement conditions. Similar conclusion emerge from research into RF; when involvement is high, regulatory focus fit may not occur (Aaker and Lee 2006; Wang and Lee 2006), because respondents may be aware of the source of the fit. We do not collect any involvement or motivation measures, so we cannot indicate the role of this construct in influencing the study effects conclusively. Further research that addresses this limitation would be very valuable.

Fourth, recent research in the public health sector (Fuhrer et al 2003) suggests that the psychosocial work environment (i.e., psychological and social conditions people experience in the workplace) plays an important role in explaining people's health status. However, we do not know whether our findings can be generalized to LPs administered by public health and fitness centers in general. Further research should investigate whether this factor influences people's regulatory focus, which may in turn affect behaviors involved in adopting LP rewards.

Fifth and finally, we cannot determine whether our findings generalize across cul-

tures. For example, people in Eastern cultures generally are more prevention oriented, whereas those in Western cultures tend to be more promotion focused (Aaker and Lee 2001). Further research should test empirically whether our findings apply across cultures.

\section{Managerial Implications}

Our findings suggest several managerial implications. First, the type of reward and type of message framing jointly determine reward value perceptions. Therefore, managers administering LPs should focus on a careful and deliberate positioning of the program rewards. If a reward can be associated with a prevention or promotion focus, communicating it using loss or gain frames will enhance its value. That is, if management decides to offer a promotiontype reward, they should communicate the benefits of attaining it by emphasizing promotion or achievement goals and focus primarily on promotion-focused members. However, if LP managers want to offer a prevention-type reward, they should communicate its benefits by emphasizing prevention or avoidance goals and focusing primarily on prevention-focused members. Because exercising commonly is associated with prevention behaviors, members likely will be attracted more to prevention-type rewards.

Second, it seems important to pay attention to program members' regulatory disposition, which also influences their reward choices. Specifically, as a personality trait, a prevention focus has a positive impact on the choice of a prevention-type reward, whereas a promotion focus has a positive influence on the choice of a promotion-type reward. Using a short questionnaire, managers could determine members' regulatory orientations when they first sign up for the LP, which would avoid any situational influences associated with the program. 
Third, positioning rewards in terms of prevention and promotion concerns and gain and loss frames offers a promising and useful strategy for attracting new LP members, retaining existing LP members, and contributing to the overall success of a LP. 


\section{CHAPTER 4}

\section{Take It or Leave It: \\ The Impact of Regulatory Fit on Reward Redemption in Channel Reward Programs}

Two studies demonstrate that regulatory focus influences the choice of rewards in a loyalty program designed to stimulate repetitive behavior and member loyalty in a channel reward program (CRP). In the first study, the authors find that the interaction between the type of reward and the type of payoff represents an important determinant of perceived value, investment decisions, and program loyalty. The second study reveals that the interaction between the type of reward, the type of payoff, and the communication of the payoff (i.e., verbal or numerical) influences response measures. These findings indicate that matching the format of payoff presentations with aspects of regulatory focus may enhance the effectiveness of CRPs. 


\section{Introduction}

The popularity of loyalty programs in business-to-consumers (B-to-C) markets, such as airlines, hotel, and even coffee industries, seems to have inspired supplier-customer relationship management in business-to-business (B-to-B) markets as well. Channel rewards programs (CRPs) or retail loyalty programs, in which retail partners participate in suppliersponsored loyalty programs, currently enjoy rapidly growing popularity (e.g., Chang 2006; Loro 1998). Capizzi and Ferguson (2005) report that nearly half of Canadian and U.S. retailers belong to a CRP initiated by one of their suppliers. For example, computer supplier CompUSA and lens manufacturer Essilor use CRPs to stimulate sales and strengthen relationships with their networks of independent resellers. Unlike consumer loyalty programs, CRPs typically include marketing support initiatives, such as lead generation and database maintenance assistance or employee training modules. Companies such as IBM, HewlettPackard, and Cisco offer comprehensive sets of rewards to their channel partners, ranging from training credits to data warehousing and certification. Frequently, these rewards are designed and tailored to the specific needs of CRP members to ensure an optimal return on the investment (ROI) in program points. Moreover, CRPs often require investments of time, effort, and transaction costs on the part of the retailer. Therefore, CRP members' investment decisions regarding spending points earned likely depend on their assessment of the ROI associated with the reward, that is, the trade off between potential gains and investment costs. However, common criticisms directed at CRPs by members include that the rewards offered do not always match retailers' strategic business needs or the payoff of program rewards remains unclear (Schwartz, Scannell, and McEachern 2006). Moreover, retailers may be reluctant to belong to a CRP designed to tie them too exclusively to a particular manufacturer (Chang 2006). Thus, practitioners need an in-depth understanding of how CRP rewards can be positioned best in terms of potential payoff and how this positioning affects members' commitment to the program.

We employ regulatory focus (RF) theory as a theoretical basis for explaining retailers' perceptions of the value of CRP rewards and their decision to redeem program points. The theory's central tenet is that two fundamental motivational foci govern people's decisions and behavior: (1) a promotion focus, which aims to achieve positive outcomes, and (2) a prevention focus, directed at avoiding negative outcomes (Higgins 1997). A decision outcome gets evaluated more favorably when the focus matches the provided information (Aaker and Lee 2006; Avnet and Higgins 2006; Higgins 2002). This outcome--value effect, or more generically regulatory fit (Aaker and Lee 2006), suggests that retailers perceive CRP as more valuable and will be more inclined to redeem points when information about the payoff is presented in a way that aligns with their regulatory focus. Thus, retailers with a service excellence program likely find more value in a service training module, whereas 
promotion-focused retailers likely favor a reward program that centers on lead generation. In attempting to confirm this prediction for CRPs, we make three substantive contributions.

First, CRP reward redemption decisions involve two types of uncertainty: (1) about the type of rewards that can and cannot be obtained as a result of point investments and (2) about the ROI or payoff of the chosen reward. Although predictions about how people process gain and loss information have been well documented in RF studies in recent decades (for an overview, see Higgins and Spiegel 2004), a relative paucity of information indicates how such processing may be influenced by information about reward payoffs. Therefore, we contribute to RF literature by examining how the type of CRP reward and information about payoffs, framed as the magnitude of the payoff multiplied by its probability of occurrence (e.g., $80 \%$ chance of gaining a $15 \%$ increase in annual profits) may jointly influence retailers' perceptions of the value of the reward, their redemption decision, and their subsequent CRP commitment.

Second, diverging opinions in the literature describe the most effective presentation format for uncertain outcomes, such as potential payoffs. Von Winterfeld and Edwards (1986) argue that people prefer numerical information because it leaves less room for ambiguity. For example, most people prefer numerical to verbal information when learning about the potential for medication side effects (e.g., .01-.1\% versus these effects occur very rarely) (Knapp, Raynor, and Berry 2004). In contrast, Windschilt and Wells (1996) and Budescu, Weinberg, and Wallsten (1988) posit that a verbatim presentation of outcomes (e.g., it is very likely that the potential payoff is very high) is more convincing because it fits with the context of communicating uncertainty better. Previous RF research further reveals that people's reactions to abstract and concrete information depend on their own regulatory focus (Semin, Higgins, and Montes 2005). Because the presentation format is relevant for the positioning, perceived value, and choice of CRP rewards, we contribute to the literature by examining the interaction between numerical and verbal reward payoff structures.

Third, RF theory conceptualizes promotion and prevention foci as situational factors (i.e., states) as well as personality characteristics (i.e., traits). Framing RF as a state may dominate RF as a trait, such that the outcome reflects the experimental manipulation, regardless of the personal orientation (e.g., Higgins 1997, 2000). Although state foci may be specific to the task and context of an experiment, this view seems to neglect people's different levels of RF strength and their multiple goals, which vary according to their orientation. As a measure of the domination of state RF over trait RF in an experimental setting, previous research has relied solely on items that serve as manipulation checks, which seems to ignore that trait RF, as a general dispositional variable, may be quite stable over time as well as in the context of experimentally designed stimuli. In extant goal orientation (GO) research, for example, trait GO has a significant and unique influence on outcome variables, independent of state or situationally induced GO (Breland and Donovan 2005; Kozlowksi et al. 2001). Because CRPs are particularly suitable to relatively large-scale segments of small 
and medium-sized businesses, in which most decisions depend directly on the personal orientations of the entrepreneurs (Day, Reynolds, and Lancaster 2006), it seems important to examine whether different ways of positioning CRPs overshadow retailers' regulatory orientation, or whether each positioning has a distinct influence on value perceptions and investment decisions. Thus, we extend prior RF research by examining the simultaneous impact of state and trait RF within a single experimental design.

\section{Conceptual Underpinnings and Hypotheses}

Faced with investment options, decision makers frequently obtain payoff information from the decisions they have made in the past (Grosskopf, Erev, and Yechiam 2006). Investor decision making in stock markets commonly results from the performance of personal mutual funds, along with other funds. Moreover, investors often rely on financial advisors and peer-to-peer information. However, when new, innovative rewards appear in a CRP, channel partners must rely on payoff information presented by the program designers. Consequently, they face a relatively large degree of uncertainty in their CRP reward redemption decisions. Making a decision under uncertainty entails risky consequences, because retailers have no prior experience with the actual payoff, and the forgone payoff is not known. In such a situation, a rational choice model (e.g., expected utility theory; see Keeney and Raifa 1976) suggests that channel partners will invest in the reward that provides the maximum payoff according to available information, independent of how the information is conveyed. However, prospect theory (Kahneman and Tversky 1979, 1984) proves the invalidity of this assumption, in that the way information is framed affects judgments and decisions. Yet to date, prospect theory has not been able to explain how the format of information may affect

judgments. We contend that a motivational orientation, such as regulatory foci, may affect redemption decisions as well.

Thus, the central premise of this research is that promotion- and prevention-focused retailers differ in their sensitivities to the type of uncertainty information, according to its phrasing in terms of the level of payoff and presentation formats. In the next sections, we develop hypotheses addressing how retailers' regulatory focus, as a result of their reward evaluation (i.e., state RF), influence their responses to this type of information. At the same time, we examine the impact of both these responses and the retailer's trait RF on the perceived value of the reward and redemption decisions.

\section{Regulatory Fit, Perceived Value, and Redemption Decisions}

Building on RF theory, we predict that retailers that evaluate rewards with high potential payoffs are more likely to invest in those rewards when the rewards emphasize promotion rather than prevention concerns. We base this prediction on evidence that retailers evaluating promotion (prevention) rewards exhibit a promotion (prevention)-focused state 
(Mourali, Bockenholt and Laroche 2007; Zhou and Pham 2004). Because a promotion focus is characterized by sensitivities to high potential gains and ensuring hits (i.e., not wanting to miss an opportunity), promotion-focused retailers should prefer to invest in rewards with high potential payoff. In contrast, prevention-focused retailers tend to want to minimize negative outcomes, such as losses or low payoffs, and therefore will be more vigilant in attempting to prevent failure in realizing payoffs. Promotion-focused retailers feel more guilty about an error of omission (e.g., forgoing a good offer), whereas prevention-focused retailers feel more guilty about an error of commission (e.g., investing in a bad option) (Camacho, Higgins, and Luger 2003).

According to prior research, promotion and prevention foci also differ in terms of the propensity toward a status quo (Chernev 2004; Liberman et al. 1999), such that preference for the status quo is more pronounced among prevention-focused than among promotionfocused persons. A prevention focus, relative to a promotion focus, is more likely to weigh the negative consequences of any potential departure from the status quo more heavily (i.e., inaction is preferred over any actions). The well-known loss aversion principle also implies that disadvantages associated with present investment opportunities often outweigh their advantages, which is more salient with a prevention focus.

Despite these findings, these research conclusions are based on experimental studies in which (1) investing entails an effort-free activity, such that investment ability is not an issue, and (2) investment activities are presented as one-time decisions. Such assumptions hardly seem realistic for gaining in-depth insights into retailers' reward redemption within a CRP context, because point investment requires retailers to engage in efforts to accumulate points and because their future investment activities typically depend on the features of their previous investment (e.g., amount of points spent to earn rewards, type of rewards). Thus, with respect to evaluating low payoffs, retailers with promotion and prevention rewards may perceive that the opportunity is proximate to the status quo described by the current profitability situation. In turn, they should be more inclined to hold their CRP points to engage in a potential, attractive future investment opportunity, regardless of the type of the present reward. Accordingly, we predict:

$\mathrm{H}_{1 \mathrm{a}}$ : The perceived value of a promotion- and prevention-type reward is equal when the payoff is low. In contrast, the perceived value of a promotion-type reward is higher than the perceived value of a prevention-type reward when the payoff is high.

$\mathrm{H}_{1 \mathrm{~b}}$ : The likelihood of redemption among retailers that evaluate promotion- and preventiontype rewards is equal when the payoff is low. Retailers that evaluate a promotiontype reward are more likely to invest in that reward than retailers who evaluate a prevention-type reward when the payoff is high. 


\section{RF as a Trait}

Previous empirical studies examine the influence of RF on behavior using a series of related yet separate experimental designs. Typically, they assess state RF with a priming technique in one experiment and trait RF with a self-reported measure in another experiment (e.g., Higgins et al. 2001; Lockwood, Jordan, and Kunda 2002). We know of no empirical research that addresses the effect of state and trait RF simultaneously in a single study. The rationale and assumption behind this approach states that when RF is manipulated situationally, the priming effect typically dominates the trait (Higgins 2000). However, to the best of our knowledge, no empirical justification exists for this assumption. Therefore, we incorporate RF as a retailer's dispositional trait to assess simultaneously, rather than independently, the relative contributions of situational and dispositional foci. In our B-to-B context, point investment decisions by CRP members may be determined by both situational and individual differences. For example, retailers may differ in their tendency to invest according to their ability to bear the negative consequences if the reward underperforms (e.g., too much time and cost expended with inadequate ROI). Whether reward implementation succeeds also depends on situational factors, such as the customer's reaction to a competitor's offer. In this case, CRP rewards that help retailers implement promotional offerings would not attract new customers if a competitor's offers are more attractive. In B-to-C reward programs (e.g., Taylor and Neslin 2005), evidence exists of such a simultaneous impact of situational factors and customer characteristics on reward redemption behavior.

Consistent with recent theorizing in social psychology, we argue that both types of RF influence respondents' attitudes and behavior. Our prediction that the priming effect of the reward will not entirely dominate the RF strength of the retailers draws on two rationales. First, retailers are entrepreneurs that have internalized specific foci as a result of their entrepreneurial activities, such as generating new market opportunities (Brockner and Higgins 2004). Second, in a field experimental setting, the impact of situational priming may differ from that in a laboratory setting, as is traditionally used (Head et al. 1988).

Prior research on RF theory demonstrates that promotion-focused people tend to use an eagerness strategy, ensure hits, avoid errors of omission, adopt to changes, generate alternatives, and prefer action over inaction. In contrast, prevention-focused people are likely to use a vigilant strategy, try to stick to the same alternatives or initial strategy, and prefer inaction to action (Chernev 2004; Crowe and Higgins 1997; Liberman et al. 1999; Higgins 2002). Thus, a decision to redeem points should be the preferred action among promotionfocused retailers. Previous RF research shows that investment decisions can be conceived of as the willingness to adopt change (invest) or the status quo (not invest) (Chernev 2004; Liberman et al. 1999). Investments represent a form of entrepreneurial activity that requires considering new possibilities and looking for new alternatives (Brockner and Higgins 2004). Therefore, we hypothesize:

$\mathrm{H}_{2}$ : A retailer's promotion focus relates positively to its investment decisions. 
$\mathrm{H}_{3}$ : A retailer's prevention focus relates negatively to its investment decisions.

Prior RF research also offers little guidance about the influence of dispositional RF on value perceptions. What does become clear, however, is that promotion and prevention foci influence how a person processes information related to abstract (e.g., happiness, security) or concrete (e.g., earning money, collecting CRP points) constructs. Similarly, the two foci affect processing of global or holistic perceptions of an object versus its constituent parts (Forster and Higgins 2005). For example, Forster and Higgins (2005) report that global processing is facilitated by promotion rather than prevention foci, whereas local processing aligns better with prevention focus, because prevention-focused people are more concerned with security and vigilant in maintaining this security. Therefore, their inclination is to focus on concrete details to eliminate obstacles to their goal fulfillment. In the case of promotionfocused people who emphasize growth and advancement through an eagerness strategy, attention to detail creates perceived inefficiencies (Forster and Higgins 2005). To fulfill their needs, promotion-oriented people tend to focus on abstract, global issues (Higgins 2002; Semin, Higgins, and Montes 2005). Because value perceptions entail global processing and abstract concepts (hedonic or utilitarian), they should be facilitated by a retailer's promotion rather than prevention focus. Thus, we hypothesize:

$\mathrm{H}_{4}$ : A retailer's promotion focus relates positively to value perceptions.

$\mathrm{H}_{5}$ : A retailer's prevention focus relates negatively to value perceptions.

\section{Value Perceptions, Investment Decisions, and CRP Loyalty}

Rewards in our CRP context typically include intangible offers that have direct benefits for improving the business of the CRP members (e.g., training, direct mail program). Because of their relevance to the retailer's business, we expect that stronger value perceptions of the reward should lead to investment decisions. We hypothesize:

$\mathrm{H}_{6}$. Value perceptions relate positively to investment decisions.

Previous loyalty research reveals a positive relationship between reward perceptions and the attractiveness of the loyalty program (Yi and Jeon 2003; Kivetz and Simonson 2002, 2003), which mirrors Rothschild and Gaidis's (1981) prediction that many loyalty programs induce loyalty to the program rather than to the product if customers have an intrinsic interest in the product. In our context, this assertion also should hold, because retailers that derive value perceptions from the reward (if it is in line with their orientation and marketing tactics) attribute value perceptions to the CRP. In other words, retailers translate value perceptions into program loyalty, such that retailers with higher rather than lower value perceptions likely are more loyal to the CRP. We hypothesize about this relationship as follows: 
$\mathrm{H}_{7}$ : Value perceptions relate positively to CRP loyalty.

Investment decisions also should have a positive relationship with CRP loyalty, for two main reasons. First, CRP rewards can be obtained only if retailers invest certain resources (e.g., purchase manufacturer's products or services). From a cost-benefit perspective, the retailer incurs no costs from not participating in the CRP, as long as the requirement for joining the CRP is not perceived as having negative consequences. Second, the CRP supplier functions as a business partner, which helps retailers implement the rewards. From a resource investment theory perspective (e.g., Morais, Dorsch, and Backman 2004), the supplier's involvement in successful reward implementation helps maintain the retailer's loyalty toward the CRP. Thus, we expect that retailers that invest in a reward after earning a sufficient number of points are likely to be more loyal than those who do not invest.

$\mathrm{H}_{8}$ : A retailer's investment decisions relate positively to CRP loyalty.

We present our conceptual framework in Figure 1

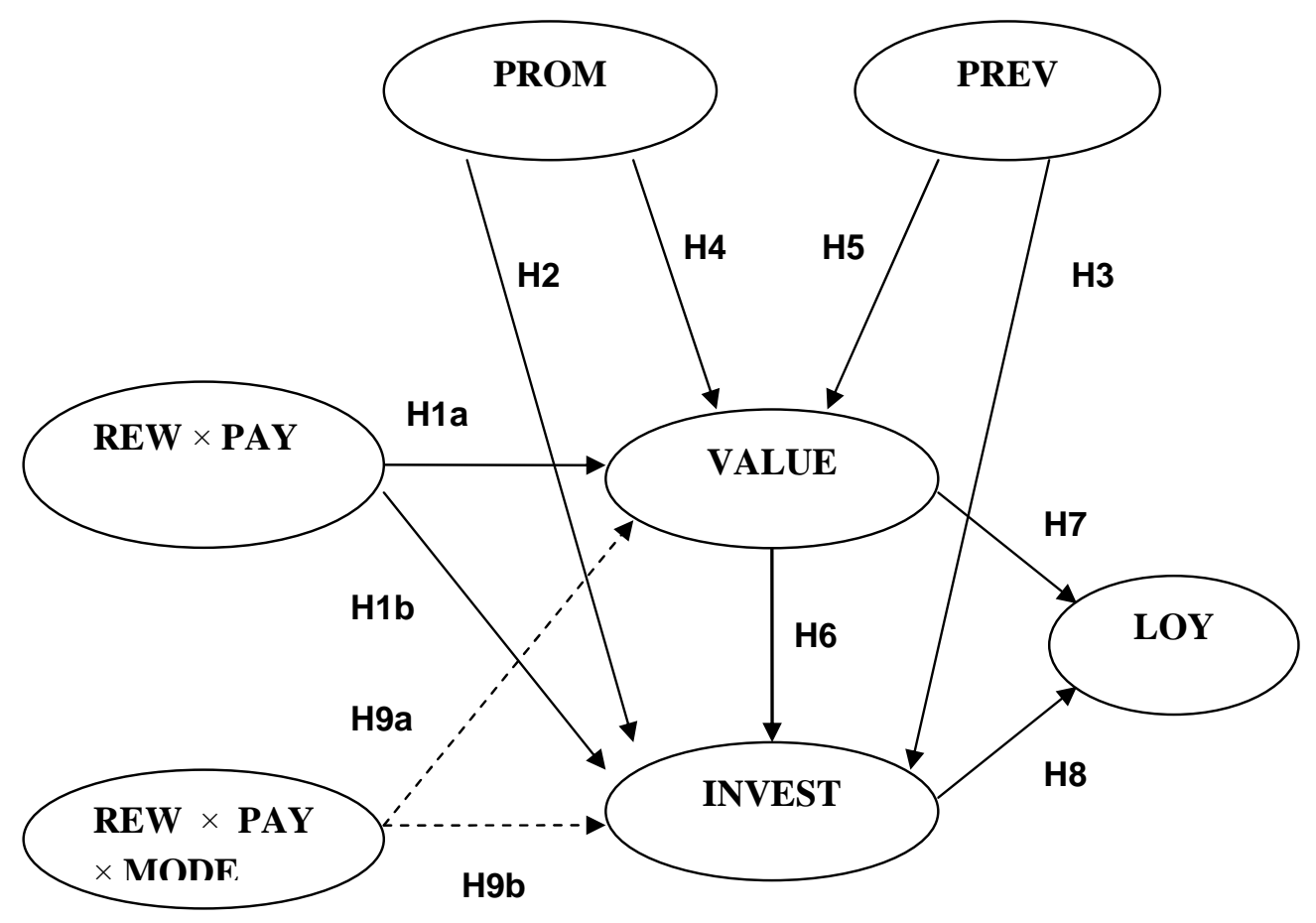

Notes: REW = type of reward; PAY = level of payoff; FORM = type of presentation format; PROM = promotion focus; PREV = prevention focus; VALUE = perceived value, INVEST = investment intention, and LOY = CRP loyalty. Dashed lines indicate hypotheses examined in Study 2.

Figure 1: Conceptual Framework 


\section{STUDY 1}

\section{Research Setting}

We conduct our study among independent optical retailers in the Netherlands. In this industry, reward and incentive programs are relatively common. Most manufacturers in this market have innovative sales and service support programs that retailers can redeem by accumulating credits based on their purchase of specified quantities of products (e.g., lenses, contact lenses, frames, accessories). We obtain the names and addresses of optical retail stores from the database of an optician's trade organization.

\section{Experimental Design}

To test our hypotheses, we design a $2 \times 2$ between-subjects experiment in which we manipulate the type of reward (direct mail program versus customer service training) and the expected payoff (low versus high). We randomly assign the respondents to one of the four scenarios and then collect the data using a questionnaire that contains general instructions, scenario descriptions, and a list of items that measure RF, value perceptions, investment decisions, and CRP loyalty. In addition, the questionnaire features several demographic measures, such as age, gender, and average number of employees per store, as well as loyalty program membership length.

\section{Procedure and Scenarios}

The scenarios asked the retailers to imagine that they had acquired 2000 credit points in a CRP and subsequently received a new opportunity to spend these credit points on either customer service training (prevention reward) or direct-mail (promotion reward) programs. Respondents also received diverging information with respect to the payoff. We include a sample scenario as an illustration of the manipulations in Appendix B.1.

\section{Pretest}

To assess the qualitative judgment of the rewards (direct mail versus customer service train-

ing), we recruited eight judges from a network of professional marketing consultants who had an affinity with the market and worked with the trade organization. The judges knew the definitions of promotion and prevention strategies, derived from RF literature, and reviewed a list of marketing strategies that included direct mail and customer service training. Then, they classified each strategy into one of four categories, namely, promotion, prevention, both, or neither. To check the reliability and proportion of agreement among the 
judges, we use the proportional reduction in loss (PRL) reliability measure (Rust and Cooil, 1994). For both direct mail and customer service training, seven of the eight judges correctly categorized the marketing strategy as a promotion or prevention strategy (interjudge agreement $=.78, \mathrm{PRL}=1.00$; Rust and Cooil 1994). This reliability score gives us sufficient

confidence that we can use direct mail and customer training as proxies for promotion and prevention rewards.

We administer another pretest to 27 opticians to check the ecological validity of the scenarios and whether they perceived the manipulation of the expected payoff and rewards as we intended. From this pretest, we discovered that we needed to adapt the manipulation of expected payoff. Therefore, in the final version of the scenario, we specify the high payoff as an $80 \%$ chance of gaining a $15 \%$ increase in annual revenues, for a total expected value of $12 \%$. For the low payoff, we describe a $30 \%$ chance of gaining a $4 \%$ annual increase for an expected value of $1.2 \%$. The pretest reveals no additional obstacles.

\section{Measurement Issues}

In addition to the experimental factors, we include four constructs in our model: value perceptions, investment decisions, CRP loyalty, and RF. All constructs rely on seven-point Likert scales anchored by strongly disagree (1) and strongly agree (7). Value perceptions, loyalty, and RF employ multiple items, and investment decisions uses three items. We adapt the specific items to the characteristics of the research setting and scenario.

To measure trait RF, we adapt a scale developed by Lockwood, Jordan, and Kunda (2002) to capture individual goal-specific orientations on the basis of promotion-ideal and prevention-ought goals. The scale consists of two subscales designed to measure promotion goal strength and prevention goal strength. The items used to measure loyalty are based on previous research by Yi and Jeon (2003) and McMullan and Gilmore (2003). We also use three items to tap the loyalty construct. For the measure of value perceptions, we use three items based on the scale developed by Yi and Jeon (2003). Finally, we develop a three-item measure for investment decisions. We list these measurement items in Table 1.

In the questionnaire, we include items that serve as manipulation checks. For the manipulation of reward type, we use different items for each type of reward, rated on sevenpoint Likert scales from strongly disagree (1) to strongly agree (7). For the direct-mail and training reward scenarios, the items are: "I see this consumer direct-mail [customer service training] program as a way to win new costumers" and "I see this consumer direct-mail [customer service training] program as a way to enhance our service orientation and customer loyalty." To manipulate payoff, we use one item- "The increase in revenue I will earn from investing in the reward is..."-measured on a nine-point bipolar scale anchored by low (=1) and high (=9). 


\section{Data Collection}

We sent the questionnaires for Study 1 to 500 optical retailers, and the trade organization conducted several follow-up e-mails and telephone calls. These efforts yielded a return of 120 questionnaires, though 3 responses were incomplete, so we obtain a net sample of 117 (response rate: $23 \%$ ). The following demographic profile emerges from the sample: $84.6 \%$ of the respondents were men, and $65.8 \%$ were aged between 26 and 45 years. Forty-nine percent of respondents had been members of a loyalty program for 24 months or longer, and $88.0 \%$ ran a one-store operation. Of the respondents, $23.1 \%$ hire one employee only, $19.7 \%$ have two employees, $31.6 \%$ have three to five employees, and $22.2 \%$ employ more than five people.

\section{Manipulation Checks}

With regard to the reward type, we find that in the direct mail scenario, respondents reveal a higher mean score for the promotion focus ("a way to win more customers"; $\mathrm{M}=5.09$, SD $=0.75$ ) than the prevention focus ("a way to enhance our service orientation and customer loyalty"; $\mathrm{M}=3.16, \mathrm{SD}=.80)$. In the training scenario, respondents show a lower mean score for the promotion focus $(\mathrm{M}=3.18, \mathrm{SD}=.74)$ than the prevention focus $(\mathrm{M}=5.26, \mathrm{SD}$ $=.73)$. Moreover, in both scenarios, we find a significant result of prevention $(\mathrm{t}(115)=$ $14.84, p<.001)$ and promotion $(\mathrm{t}(115)=13.87, p<0.001)$ foci. Our results also indicate a significant difference $(t(115)=29.57, p<.001)$ in the manipulations checks of payoff $\left(\mathrm{M}_{\mathrm{LOW}}=3.21, \mathrm{SD}=.75 ; \mathrm{M}_{\mathrm{HIGH}}=7.25, \mathrm{SD}=.73\right)$, which indicates that the manipulations of the scenarios produce the intended effects (Perdue and Summers, 1986). 
Table 1: Measurement Instruments (Study 1)

\begin{tabular}{|c|c|}
\hline Items & $\begin{array}{l}\text { Standardized } \\
\text { Loading }\end{array}$ \\
\hline \multicolumn{2}{|l|}{ Value Perception } \\
\hline The credit-points in the CRP represent a high cash value to me. & 0.887 \\
\hline $\begin{array}{l}\text { The range of options I have of investing my investing my credit-points in sales- and service- } \\
\text { support is of great value to me. }\end{array}$ & 0.802 \\
\hline The service and sales support that I can obtain represent rewards that I would like to have. & 0.817 \\
\hline \multicolumn{2}{|l|}{ Investment Intention } \\
\hline I will most likely invest my accumulated credit points in this project for next year. & 0.887 \\
\hline $\begin{array}{l}\text { I will most probably recommend to a fellow participant in the program to invest his/her accu- } \\
\text { mulated credit points in this project for next year. }\end{array}$ & 0.884 \\
\hline It is most unlikely that I will invest my accumulated credit points in this project for next year. & 0.891 \\
\hline \multicolumn{2}{|l|}{ Program Loyalty } \\
\hline I appreciate the CRP. & 0.853 \\
\hline I have a strong preference for this particular CRP because the program is appealing. & 0.898 \\
\hline I would recommend this CRP to others. & 0.861 \\
\hline \multicolumn{2}{|l|}{ Promotion Focus } \\
\hline I am frequently concerned with how I can achieve my hopes and aspirations. & 0.799 \\
\hline I often think about the person I would ideally like to be in the future. & 0.837 \\
\hline I typically focus on the success that I hope to achieve in the future & 0.801 \\
\hline My major goal in business right now is to achieve my business ambition. & 0.855 \\
\hline In general, I am focused on achieving positive outcomes in my life. & 0.788 \\
\hline \multicolumn{2}{|l|}{ Prevention Focus } \\
\hline In general, I am focused on preventing negative events in my life. & 0.822 \\
\hline I am anxious that I will fall short of my responsibilities and obligations.. & 0.863 \\
\hline I often think about the person I am afraid I might become in the future. & 0.563 \\
\hline I often worry that I fail to accomplish my business goals. & 0.879 \\
\hline I often imagine myself experiencing bad things that I fear might happen to me. & 0.851 \\
\hline I am more oriented toward preventing losses than I am toward achieving gains. & 0.839 \\
\hline
\end{tabular}

\section{Data Analysis}

We use partial least squares (PLS) path modeling to test the hypotheses; specifically, we employ PLS-Graph software version 3.0 (Chin 2001) to obtain the estimates for the parameters in the structural and measurement models. Because PLS is a component-based structural model, it has several advantages over traditional covariance-based structural equation modeling (SEM) analysis (e.g., LISREL, EQS). For example, PLS does not require multivariate normal data, is suitable for small samples, and places minimum requirements on measurement scales (Chin 1998; Hulland 1999; Tenenhaus et al. 2005; Wold 2001). We 
obtain parameter estimates of the model PLS with an iterative estimation algorithm that consists of a series of simple or multiple ordinary least squares analyses (Chin 1998). Moreover, PLS modeling is more appropriate for modeling complex construct relationships with large numbers of observed variables than can be modeled as formative indicators. In addition, PLS can model factorial designs and has several clear advantages over traditional experimental designs, including its ability to control for measurement error and its greater flexibility in modeling, as well as its capabilities for examining the treatment manipulation while controlling for covariates related to that treatment manipulation (Mackenzie 2001).

\section{Measurement Model}

We use PLS path modeling to analyze the $2 \times 2$ between-subject experimental design. The sample sizes of the four cells are $28,29,33$, and 27 . We specify single indicators for the manipulated factors, types of reward and expected payoff, and the interaction effect and code the manipulations using effect coding, such that high payoff is coded as 1 and low payoff as -1 , and direct mail is 1 and training is -1 . The use of effect coding for categorical predictors in a multiple regression analysis of factorial designs is preferable to dummy coding $(0,1)$, because it produces tests of the main effects and interactions for any models that involve interaction terms (O'Grady and Medoff 1988; Pedhazur 1997). Therefore, testing coefficient estimates for main and interaction effects is valid with effect coding but not with dummy coding (Pedhazur 1997).

We specify the interaction between payoff and reward as the product term of the two effect-coded variables (payoff $\times$ reward). The promotion focus and prevention focus subscales appear as latent variables with nine indicators each. However, in the analysis, we delete four indicators for promotion and three for prevention because their loadings are below the cut-off value of .50. Thus, our final model contains five items that tap the promotion focus and six items that relate to the prevention focus. The dependent variable, investment decisions, uses three items, specified as reflective indicators. The three loyalty items and three value perception items also represent reflective indicators. We examine the reliability, convergent validity, and discriminant validity of the reflective indicators for value perceptions, loyalty, promotion, and prevention.

In Table 5, we provide the PLS parameter estimates for the measurement model. To measure the internal consistency of a given block of indicators, w e employ composite reliability (CR) and average variance extracted (AVE; Fornell and Larcker 1981). As we reveal in Table 2, the CR scores for value perceptions, investment decisions, and loyalty are .874 , .917 and .904 , respectively. The CRs for the promotion and prevention constructs are .910 and .918 , respectively, which exceed the cut-off value of .7 proposed by Nunnally and Bernstein (1994). The AVEs for value, invest, and loyalty are $.699, .787$, and .759, respectively, which again exceed the cut-off value of .5 suggested by Fornell and Larcker (1981). 
To assess the discriminant validity for each construct, we apply three methods. First, a construct should share more variance with its measures than it shares with other constructs (Chin 1998, Hulland 1999). Therefore, the square root of the AVE should exceed the intercorrelations of the construct with other constructs in the models. In our study, the square root of the AVE for all constructs is greater than any corresponding correlations (Fornell and Larcker 1981). Second, cross-loadings with constructs other than those hypothesised are not substantial in magnitude (<.3; Chin 1998; Hulland 1999). Third, the correlations of the residual terms across blocks do not exceed |.2| (Falk and Miller 1992; Fornell and Bookstein 1982). Therefore, we conclude that all constructs exhibit satisfactory discriminant validity. We inspect the constructs' factor loadings to assess their convergent validity and, as we show in Tables 1 and 2, the factor loadings for value, invest, loyalty, promotion, and prevention are greater than .50 , in support of acceptable convergent validity.

Table 2: Means, Standard Deviations, Composite Reliabilities, and Correlations among Construct Scores (Study 1)

\begin{tabular}{lllllllllll}
\hline Construct & CR & AVE & $\mathbf{1 .}$ & $\mathbf{2 .}$ & $\mathbf{3 .}$ & $\mathbf{4 .}$ & $\mathbf{5 .}$ & $\mathbf{6 .}$ & $\mathbf{7 .}$ & $\mathbf{8 .}$ \\
\hline & & & & & & & & & & \\
1. PAY & na & na & na & & & & & & & \\
2. REW & na & na & -0.059 & Na & & & & & & \\
3. PAY*REW & na & na & -0.041 & 0.023 & $\boldsymbol{n a}$ & & & & & \\
4. VALUE & 0.874 & 0.699 & 0.514 & 0.249 & 0.139 & $\mathbf{0 . 8 4 6}$ & & & & \\
5. INVEST & 0.917 & 0.787 & 0.337 & 0.340 & 0.236 & 0.504 & $\mathbf{0 . 8 8 7}$ & & & \\
6. LOY & 0.904 & 0.759 & 0.424 & 0.156 & 0.154 & 0.511 & 0.704 & $\mathbf{0 . 8 7 1}$ & & \\
7. PROM & 0.910 & 0.669 & 0.074 & -0.023 & 0.021 & 0.218 & 0.289 & 0.345 & $\mathbf{0 . 8 1 8}$ & \\
8. PREV & 0.918 & 0.657 & -0.011 & -0.070 & 0.049 & -0.153 & -0.255 & -0.088 & -0.085 & $\mathbf{0 . 8 1 1}$ \\
\hline
\end{tabular}

Notes: Average variance extracted (AVE) on diagonal. PAY = expected payoff; REW = reward type; VALUE = value perception; INVEST $=$ investment intention; $L O Y=$ program loyalty; $P R O M=$ promotion focus; $P R E V=$ prevention focus; $\mathrm{CR}=$ composite reliability.

\section{Structural Model}

We display the PLS estimates of the structural model in Table 3. These path coefficient estimates are the results of a nonparametric bootstrapping procedure with 1,000 resamples (Chin 1998). Because we do not offer hypotheses regarding the main effect, we do not present the coefficient estimates that link the main effects to the outcome measures. The results largely confirm our hypotheses. 
Table 3: Results for Structural Model (Study 1)

\begin{tabular}{llll}
\hline Hypothesis & Relationship & Coefficient & Result \\
\hline $\mathrm{H}_{1 \mathrm{a}}$ & REW x PAY $\rightarrow$ VAL & $0.175^{* *}$ & Supported \\
$\mathrm{H}_{1 \mathrm{~b}}$ & REW x PAY $\rightarrow$ INVEST & $0.209^{* *}$ & Supported \\
$\mathrm{H}_{2}$ & PROM $\rightarrow$ INVEST & $0.221^{* *}$ & Supported \\
$\mathrm{H}_{3}$ & PREV $\rightarrow$ INVEST & $-0.194^{* *}$ & Supported \\
$\mathrm{H}_{4}$ & PROM $\rightarrow$ VALUE & $0.178^{* *}$ & Supported \\
$\mathrm{H}_{5}$ & PREV $\rightarrow$ VALUE & -0.075 & Not supported \\
$\mathrm{H}_{6}$ & VALUE $\rightarrow$ INVEST & $0.192^{* *}$ & Supported \\
$\mathrm{H}_{7}$ & VALUE $\rightarrow$ LOY & $0.191^{* *}$ & Supported \\
$\mathrm{H}_{8}$ & INVEST $\rightarrow$ LOY & $0.608^{* *}$ & Supported \\
\hline Notes: $\mathrm{R}^{2}$ are as follows: VAL $=.41 ;$ INVEST $=.44 ;$ LOY $=.53 .^{* *} p<.01$.
\end{tabular}

In $\mathrm{H}_{1}$, we propose that a low payoff prompts retailers to perceive promotion- and prevention-type rewards equally, whereas a high payoff leads to higher perceived values of a promotion-type reward. Our results support this hypothesis (see Figure 2); we find a significant effect of the interaction term $(\beta=.175, p<.01)$. We also find support for H1b $(\beta=$ $.209, p<.01$ ), because in a low payoff situation, retailers who evaluate promotion- and prevention-type rewards indicate equal redemption tendencies, whereas in high payoff situations, retailers who evaluate promotion-type rewards are more likely to invest than are those who evaluate prevention-type rewards (see Figure 3 ).

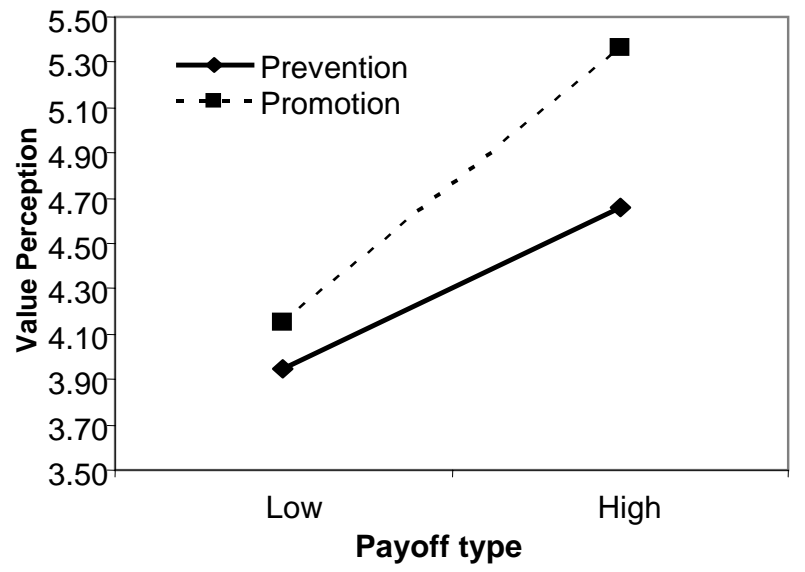

Figure 2: Interaction between Reward Type and Payoff on Perceived Value (Study 1) 


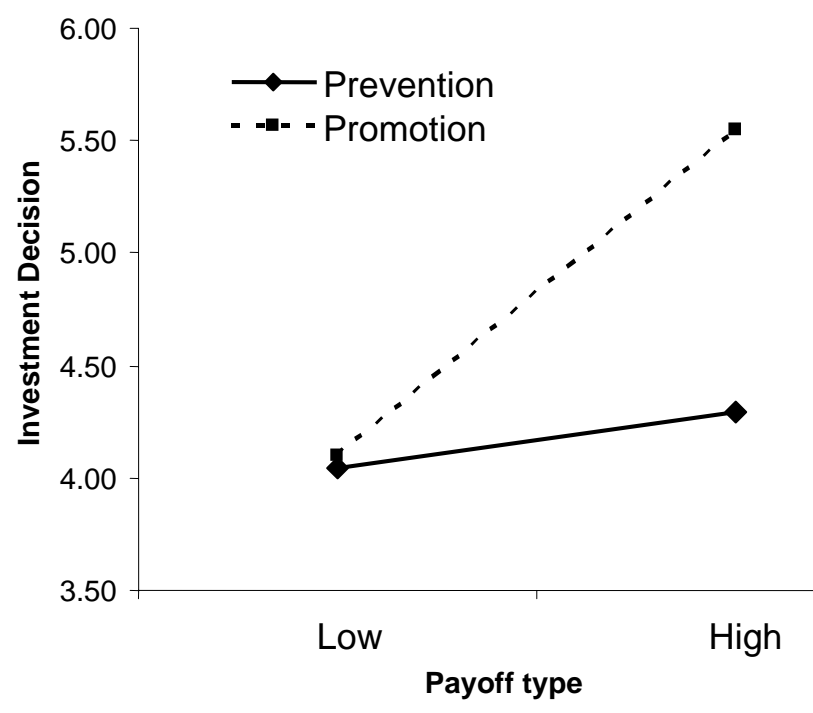

Figure 3: Interaction between Reward Type and Payoff on Investment Decision (Study 1)

We also find a positive and significant relationship between a promotion focus and the investment decision $(\beta=.221, p<.01)$ and a negative and significant relationship between a prevention focus and the investment decision $(\beta=-.194, p<.01)$, in support of $\mathrm{H}_{2}$ and $\mathrm{H}_{3}$, respectively. That is, the higher the retailer's promotion focus strength, the greater its impact on the retailer's willingness to invest, whereas when the retailer's prevention strength is lower, it has a lesser impact on the retailer's willingness to invest.

We find a positive and significant relationship between a promotion focus and value perceptions $(\beta=.178, p<.01)$ and a negative and significant relationship between a prevention focus and investment decisions $(\beta=-.194, p<.01)$, in support of $\mathrm{H}_{2}$ and $\mathrm{H}_{3}$. The greater the retailer's promotion focus, the greater its impact on the retailer's willingness to invest; the lower its prevention strength, the less its impact on the retailer's willingness to invest. Independent of RF, regarding the interrelationship among value perceptions, investment decisions, and loyalty, we find a significant positive relationship between value perceptions and investment decisions $(\beta=.192, p<.01)$, in support of $\mathrm{H}_{6}$; between value perceptions and loyalty $(\beta=.191, p<.01)$, in support of $\mathrm{H}_{7}$; and between investment decisions and loyalty ( $\beta$ $=.608, p<.001)$, in support of $\mathrm{H}_{8}$.

The $\mathrm{R}^{2}$ for VALUE, INVEST, and LOYALTY are $.41, .44$, and .53, respectively, which means that the model explains $41 \%$ of the variance in value perceptions, $44 \%$ in investment decisions, and 53\% of the variance in CRP loyalty. Although PLS path modeling has no single goodness-of-fit measure, such as the $\chi^{2}$ statistics and its derived measures for covariance-based SEM, $\mathrm{R}^{2}$ values for the endogenous constructs can be used to assess model fit (Chin 1998; Tenenhaus et al. 2005). In accordance with the categorization of the $\mathrm{R}^{2}$ effect sizes (i.e., a measure of the magnitude of the treatment effect independent of sample size) by Cohen (1988, 1992; small .02; medium .13; large .26), we conclude that the effect sizes are large. Recently, Tenenhaus and colleagues (2005) developed a global fit 
measure for PLS based on $\mathrm{R}^{2}$ values (i.e., global criterion of goodness-of-fit, or GoF), computed by taking the square root of the product of the average communality, defined as the average of a squared correlation between the manifest variable and its latent variable (equals AVE in PLS), and the average $\mathrm{R}^{2}$ value for the endogenous constructs (Tenenhaus et al. 2005). On the basis of Cohen's $(1988,1992)$ categorization and using .5 as the cut-off value for communality (Fornell and Larcker 1981), we establish GoF criteria for small, medium, and large effect sizes at .1, .25, and .36. For our model, the GoF is .583, which exceeds the cut-off value of .36 for large effect size of $\mathrm{R}^{2}$.

\section{Payoff Information in Numerical and Verbal Presentation}

In Study 1, we framed uncertainty information about the payoff using numbers. However, in many cases, people refer to or are confronted with uncertainty communicated verbally (e.g., "the chance of rain is very high today"). Although these presentation formats seem relevant for the positioning and ultimate choice of CRP rewards, to our knowledge, no prior studies examine this issue. Previous empirical work on the processing of uncertainty information shows that a preference for either a verbal or a numerical format relates to the preference for either abstract or concrete information (e.g. , Budescu, Weinberg, and Wallsten 1988; Windschilt and Wells 1996). The issue of how people process abstract and concrete information also appears in the context of RF (Semin, Higgins, and Montes 2005). Because abstract information commonly is conveyed in verbal information, whereas concrete information usually appears in numerical information, we examine the interaction between RF and numerical versus verbal payoff presentations.

Semin, Higgins, and Montes (2005) demonstrate that prevention-focused people prefer concrete information because of its relation to a strategic focus on vigilance to maintain security and ought goals (i.e., duties and obligations). In contrast, promotion-focused people relate better to abstract information, which mirrors a focus on eagerness means and ideal goals (i.e., hopes and aspirations). Semin, Higgins, and Montes (2005) also reveal that promotion-focused people indicate greater intentions to exercise when the benefit of that exercise is communicated with abstract terms, such as "exercise can increase endurance levels and energy." In contrast, prevention-focused persons show a higher intention to exercise when its benefits are communicated in more concrete ways, such as "exercise can burn approximately 440 calories per hour."

Thus, payoffs presented as verbal information should be preferable among promotionfocused people, whereas those presented as numerical information should appeal to those with a prevention focus. We predict that when retailers perceive the potential payoff of a reward as high and the reward triggers a promotion focus, they will indicate a greater intention to invest and perceive more value in the reward when the payoff information is communicated verbally rather than numerically. If the reward induces a retailer's prevention 
focus, it will reveal a greater intention to invest and value the reward more when the payoff information is communicated numerically. Thus, we hypothesize:

$\mathrm{H}_{9 \mathrm{a}}$ : The perceived value of promotion- and prevention-type rewards is equal when the potential payoff is low, regardless of its presentation format. In contrast, the perceived value of a promotion- (prevention-) type reward is greater when its potential payoff is higher and it is presented verbally (numerically) rather than numerically (verbally).

$\mathrm{H}_{9 \mathrm{~b}}$ : The investment intentions of retailers that evaluate promotion- and prevention-type rewards are equal when the potential payoff is low, regardless of its presentation format. In contrast, retailers that evaluate a promotion- (prevention-) type reward are more likely to invest in that reward when its potential payoff is high and it is presented verbally (numerically) rather than numerically (verbally).

In the second study, we also incorporate trait variables with the experimental factors to ascertain the robustness of their impact on value perceptions and redemption decisions across the two experimental designs.

\section{STUDY 2}

To test our hypotheses, we design a $2 \times 2 \times 2$ between-subjects experiment in which we manipulate type of reward (direct mail program versus customer service training), potential payoff (low versus high), and the mode of presentation (verbal versus numeric). We assign respondents randomly to one of the eight scenarios. The study context and procedure are the same as those in Study 1. We draw our sample of 900 opticians (who had not participated in Study 1) from the database of the optical reseller trade organization. The questionnaire for this study contains general instructions, scenario descriptions, and items to measure RF, value perceptions, and reward redemption decisions (seven-point Likert scales, anchored by strongly disagree [1] and strongly agree [7]). We use multiple items whose wording we adapt to the characteristics of the research setting and scenario.

For the manipulation checks of reward type, we use the same items as in Study 1. We also undertake manipulation checks for the modes of payoff presentation by adapting a scale developed by Winschilt and Wells (1996) with the objective of cross-checking whether respondents perceive verbal and numerical uncertainty information in the same way. Those who viewed the numerical format (i.e., 30\% versus $80 \%$ chance) indicated their verbal interpretations of these chances on 11-point scales ranging from impossible to certain. Participants in the verbal format condition (i.e., rather unlikely versus quite likely) instead indicated their numeric interpretation of this information on 11-point scales ranging from $0 \%$ to $100 \%$. The survey in Study 2 resulted in usable data from 231 respondents, for a 
response rate of $26 \%$. We feature a sample scenario with payoff in the verbal format to illustrate the manipulations in Appendix B.2. We also include the scale we use to check the manipulation of the modes in Appendix B.3.

\section{Results}

\section{Manipulation Check}

In the direct mail scenario, respondents provide a higher mean score for the promotion focus ("a way to win more customers"; $\mathrm{M}=5.22, \mathrm{SD}=.80$ ) than the prevention focus ("a way to enhance our service orientation and customer loyalty"; $\mathrm{M}=3.21, \mathrm{SD}=.73$ ). The training scenario respondents display a lower mean score for the promotion focus $(\mathrm{M}=$ $3.09, \mathrm{SD}=.70)$ than for the prevention focus $(\mathrm{M}=5.14, \mathrm{SD}=.69)$. Moreover, for both scenarios, we find significant results for the prevention $(\mathrm{t}(229)=21.53, \mathrm{p}<.001)$ and promotion $(\mathrm{t}(229)=20.63, \mathrm{p}<.001)$ foci. With regard to the presentation modes, the results show a significant difference between low and high payoff scenarios (NUMERICAL: $\mathrm{MLOW}=3.19, \mathrm{SD}=.72, \mathrm{MHIGH}=8.15, \mathrm{SD}=.82 ; \mathrm{VERBAL}: \mathrm{MLOW}=3.23, \mathrm{SD}=.68$, $\mathrm{MHIGH}=8.29, \mathrm{SD}=.70)$ and between the numerical $(\mathrm{t}(115)=34.72, \mathrm{p}<.001)$ and verbal $(\mathrm{t}(112)=39.34, \mathrm{p}<.001)$ presentations. Thus, the manipulations produce the intended effects (Perdue and Summers, 1986).

In Table 4, we list the standardized factor loadings for the measurement model. Because the factor loadings for all constructs are greater than .50, we have support for acceptable convergent validity. In Table 5, we provide the correlations among the construct scores, $\mathrm{CR}$, and AVE, as well as the square root of the AVE. All measures exceed the cut-off values of .70 (CR) and .50 (AVE). Moreover, the square root of the AVE exceeds the intercorrelations of the construct with other constructs; therefore, all constructs exhibit satisfactory discriminant validity. Finally, in

Table 6, we provide the parameter estimates of the structural model, as well as the $\mathrm{R}^{2}$ for value perceptions, investment decisions, and loyalty, which are .547, .695, and .658, respectively.

\section{Hypotheses Testing}

The path coefficient estimates displayed in Table 6 are the results of a nonparametric bootstrapping procedure with 500 resamples (Chin 1998). As the results show, the interaction effects of reward and payoff on value perceptions and investment decisions are significant. More important, we find a significant three-way interaction effect among reward, payoff, and mode of presentation on value perceptions $(\beta=.097, p=.025)$ and investment decisions 
$(\beta=.073, p=.029)$. The plots of the cell means for the three-way interactions for value perceptions and investment decisions sppear in Figures 4 and 5, respectively.

Table 4: Measurement Instruments (Study 2)

\begin{tabular}{ll}
\hline Items & $\begin{array}{l}\text { Standardized } \\
\text { Loadings }\end{array}$ \\
\hline
\end{tabular}

\section{Value Perception}

The credit-points in the CRP represent a high cash value to me.

The range of options I have of investing my investing my credit-points in sales- and service- 0.877 support is of great value to me.

The service and sales support that I can obtain represent rewards that I would like to have.

\section{Investment Decision}

I will most likely invest my accumulated credit points in this project for next year.

I will most probably recommend to a fellow participant in the program to invest his/her accu- 0.934 mulated credit points in this project for next year.

It is most unlikely that I will invest my accumulated credit points in this project for next year.

\section{Program Loyalty}

I appreciate the CRP.

I have a strong preference for this particular CRP because the program is appealing. $\quad 0.928$

I would recommend this CRP to others.

\section{Promotion Focus}

I am frequently concerned with how I can achieve my hopes and aspirations. $\quad 0.807$

I often think about the person I would ideally like to be in the future. 0.846

I typically focus on the success that I hope to achieve in the future 0.864

My major goal in business right now is to achieve my business ambition. $\quad 0.866$

In general, I am focused on achieving positive outcomes in my life. $\quad 0.858$

\section{Prevention Focus}

In general, I am focused on preventing negative events in my life. $\quad 0.866$

I am anxious that I will fall short of my responsibilities and obligations.. $\quad 0.848$

I often think about the person I am afraid I might become in the future. 0.856

$\begin{array}{ll}\text { I often worry that I fail to accomplish my business goals. } & 0.879\end{array}$

I often imagine myself experiencing bad things that I fear might happen to me. $\quad 0.869$

I am more oriented toward preventing losses than I am toward achieving gains. $\quad 0.825$ 
Table 5: Means, Standard Deviations, Composite Reliabilities, and Correlations among Construct Scores (Study 2)

\begin{tabular}{llllllllll}
\hline Construct $^{\text {a }}$ & $\mathbf{C R}^{\mathbf{b}}$ & AVE & $\mathbf{1 .}$ & $\mathbf{2 .}$ & $\mathbf{3 .}$ & $\mathbf{4 .}$ & $\mathbf{5 .}$ & $\mathbf{6 .}$ & $\mathbf{7 .}$ \\
\hline $\begin{array}{l}\text { 1. REW x PAY } \\
\text { 2. NA }\end{array}$ & NA & 1 & & & & & & \\
PAY X MODE & NA & NA & -0.013 & 1 & & & & & \\
3. VALUE & 0.914 & 0.780 & 0.260 & 0.135 & $\mathbf{0 . 8 8 3}$ & & & & \\
4. INVEST & 0.953 & 0.871 & 0.326 & 0.142 & 0.719 & $\mathbf{0 . 9 3 3}$ & & & \\
5. LOY & 0.937 & 0.832 & 0.275 & 0.144 & 0.686 & 0.794 & $\mathbf{0 . 9 1 2}$ & & \\
6. PROM & 0.932 & 0.731 & -0.029 & -0.042 & 0.192 & 0.235 & $*$ & \\
7. PREV & 0.922 & 0.670 & -0.012 & -0.022 & -0.140 & -0.193 & -0.031 & -0.005 & $\mathbf{0 . 8 1 9}$ \\
\hline
\end{tabular}

Notes: Average variance extracted (AVE) on diagonal. PAY = expected payoff; REW = reward type; VALUE $=$ value perception; INVEST $=$ investment intention; LOY $=$ program loyalty; PROM $=$ promotion focus; $\mathrm{PREV}=$ prevention focus; $\mathrm{CR}=$ composite reliability.

Table 6: Results for Structural Model (Study 2)

\begin{tabular}{lllll}
\hline Hypothesis & Relationship & Coefficient & $\boldsymbol{p}$-Values & Result \\
\hline $\mathrm{H}_{1 \mathrm{a}}$ & REW x PAY $\rightarrow$ VAL & 0.238 & 0.000 & Supported \\
$\mathrm{H}_{1 \mathrm{~b}}$ & REW x PAY $\rightarrow$ INVEST & 0.218 & 0.000 & Supported \\
$\mathrm{H}_{2}$ & PROM $\rightarrow$ INVEST & 0.165 & 0.000 & Supported \\
$\mathrm{H}_{3}$ & PREV $\rightarrow$ INVEST & -0.138 & 0.001 & Supported \\
$\mathrm{H}_{4}$ & PROM $\rightarrow$ VALUE & 0.171 & 0.000 & Supported \\
$\mathrm{H}_{5}$ & PREV $\rightarrow$ VALUE & -0.128 & 0.008 & Supported \\
$\mathrm{H}_{6}$ & VALUE $\rightarrow$ INVEST & 0.299 & 0.000 & Supported \\
$\mathrm{H}_{7}$ & VALUE $\rightarrow$ LOY & 0.224 & 0.000 & Supported \\
$\mathrm{H}_{8}$ & INVEST $\rightarrow$ LOY & 0.632 & 0.000 & Supported \\
$\mathrm{H}_{9 \mathrm{a}}$ & REW x PAY x MODE $\rightarrow$ VAL & 0.097 & 0.025 & Supported \\
$\mathrm{H}_{9 \mathrm{~b}}$ & REW x PAY x MODE $\rightarrow$ IN- & & \\
& VEST & 0.073 & 0.029 & \\
\hline
\end{tabular}

Notes: $\mathrm{R}^{2}$ are as follows: $\mathrm{VAL}=.547 ; \mathrm{INVEST}=.695 ; \mathrm{LOY}=.658$. 


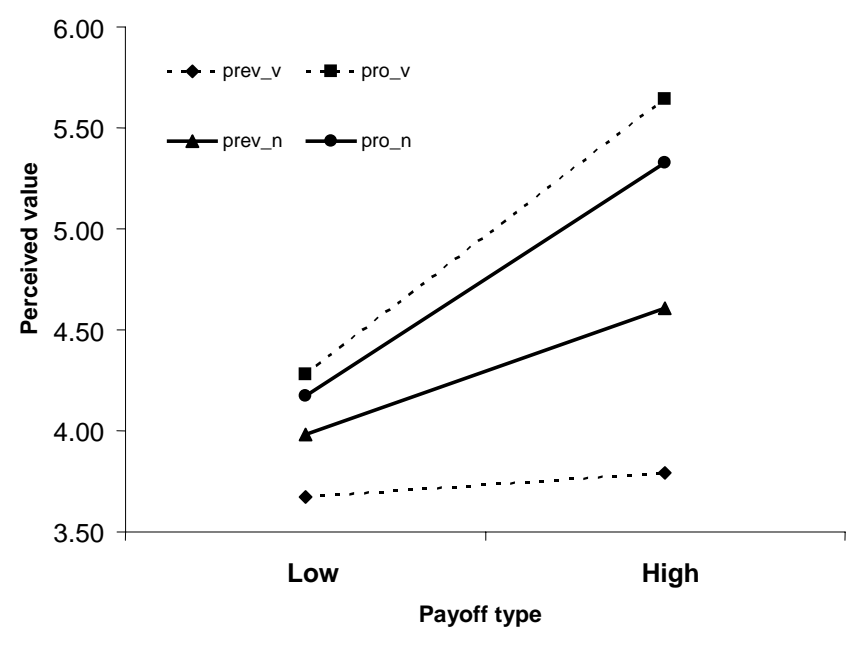

Notes: prev_v $=$ promotion reward with verbal; pro_v $=$ promotion reward with verbal; prev_n $=$ prevention reward with numerical; and pro_n $=$ promotion reward with numerical.

Figure 4: Three-Way Interaction of Reward, Payoff, and Mode on Perceived Value (Study 2)

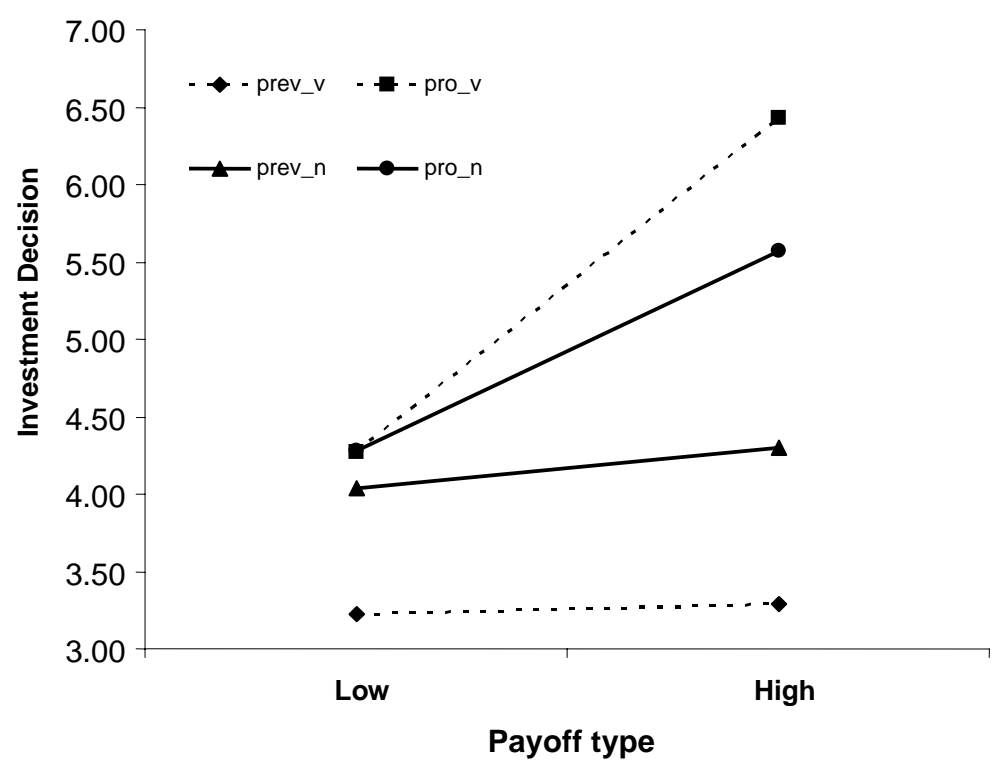

Notes: prev_v $=$ promotion reward with verbal; pro_v $=$ promotion reward with verbal; prev_ $\mathrm{n}=$ prevention reward with numerical; and pro_n $=$ promotion reward with numerical.

Figure 5: Three-Way Interaction of Reward, Payoff, and Mode on Investment Decision (Study 2)

To uncover the nature of the three-way interaction effects, we conduct an additional analysis with ANCOVA and control for the effect of promotion and prevention strength, as well as simple effect tests both for value perceptions and investment decisions. First, we examine the interaction effect of payoff and reward within each level of mode; second, we examine the interaction effect of reward and mode within each level of payoff. The result of the first 
ANCOVA shows that the interaction effect of reward and payoff on value perceptions $\left(\mathrm{H}_{1 \mathrm{a}}\right)$ and investment decisions $\left(\mathrm{H}_{1 \mathrm{~b}}\right)$ are significant for each mode level, and the simple effect analysis corroborates our findings in Study 1.

Next, we conduct an ANCOVA for each payoff level. For the high payoff condition, we find that the interaction between mode and reward is significant for value perceptions $(F(1,117)=24.132)$ and investment decisions $(F(1,117)=39.395)$. Following these significant effects, we conduct simple effect tests (Keppel and Wickens 2004) to examine the differences between verbal and numerical presentations separately for prevention and promotion rewards. Among the promotion rewards, value perceptions and investment decisions are greater in the verbal format than in the numeric format $(\mathrm{M}=5.64$ versus 5.33 for value; $\mathrm{M}=6.43$ versus 5.57 for investment). Furthermore, with prevention rewards, value perceptions and investment decisions are greater in the numerical than in the verbal format $(\mathrm{M}=$ 4.61 versus 3.79 for value; $\mathrm{M}=4.30$ versus 3.29 for investment). The results of the ANCOVA for the low payoff condition show that the interaction between mode and reward is not significant for value perceptions $(\mathrm{F}(1,102)=2.22, \mathrm{~ns})$ and is marginally significant for investment decisions $(\mathrm{F}(1,102)=3.565$, ns). Thus, regardless of the presentation formats, in low payoff conditions, the perceived value of promotion and prevention rewards are equal, as are the levels of investment decisions among retailers who evaluate the different types of rewards. These findings confirm $\mathrm{H}_{9 a}$ and $\mathrm{H}_{9 b}$.

With respect to the effect of promotion and prevention strength, we find a positive and significant relationship between promotion focus and investment decisions $(\beta=.165, p$ $<.01)$ and negative relationship between prevention focus and investment decisions $(\beta=-$ $.138, p<.01$ ); these results replicate the findings of Study 1 and support $\mathrm{H}_{2}$ and $\mathrm{H}_{3}$. That is, the higher the retailer's promotion focus strength, the greater its impact on the retailer's willingness to invest, whereas the higher its prevention focus strength, the lower its impact on willingness to invest. We also find a positive and significant relationship between promotion focus and value perception $(\beta=.171, p<.01)$ and a negative and significant relationship between prevention focus and value perceptions. Thus, the higher the retailer's promotion focus strength, the greater its impact on the perceived value of a reward, whereas the higher the retailer's prevention focus, the lower its impact. These findings again corroborate our results in Study 1 and support $\mathrm{H}_{4}$ and $\mathrm{H}_{5}$.

With respect to the interrelationship among value perceptions, intentions, and loyalty, our second study corroborates the findings of our first study. We find a significant, positive relationship between value perceptions and investment decisions $(\beta=.299, p<.01)$, in support of $\mathrm{H}_{6}$; between value perceptions and loyalty $(\beta=.224, p<.01)$, in support of $\mathrm{H}_{7}$; and between investment decisions and loyalty $(\beta=.632, p<.01)$, in support of $\mathrm{H}_{8}$. 


\section{Discussion}

With this research, we show that retailers' investment decisions are influenced by their evaluations of CRP reward information. In two experiments, we find a positive impact of the interaction between the elicitation of regulatory focus and payoff on value perceptions, as well as on redemption decisions. Moreover, we demonstrate that the use of verbal versus numerical information with regard to the payoff of the reward influences these dependent variables. Finally, our results confirm a robust impact of RF as a trait in the presence of experimentally framed foci.

In particular, the Study 1 results show that promotion-focused retailers are more likely to invest in a high payoff reward than are prevention-focused retailers, though in a low payoff condition, we find no difference in their value perceptions and redemption intentions. This finding can be interpreted in the light of a recent study by Halamish and colleagues (2007), who report that RF influences the anticipated pleasure and pain of obtaining a probable payoff. When confronted with moderate to high probability payoffs, promotion-focused people experience more pleasure in anticipating the gains than do prevention-focused people, because they hope to maximize their opportunities and therefore pay more attention to the positive consequences of realizing a certain payoff than to the negative consequences of failing to get it. The reverse situation exists among prevention-focused people, who are more oriented toward minimizing risks and failures. Alternatively, Camacho, Higgins, and Luger (2003) posit that feelings of regret resulting from past errors may account for these differential results. Promotion-focused people feel guiltier than prevention-focused people when reflecting on their errors of omission (i.e., inertia), whereas prevention-focused people are more inclined to have regrets about errors of commission (i.e., wrong choice). Therefore, promotion-focused retailers may feel more regret if they forgo a choice that implies a high payoff. Conversely, prevention-focused retailers are more likely to anticipate regret if they later discover that they have invested in a less satisfactory reward. However, in the low payoff condition, both types of retailers seem more pessimistic about the chance (i.e., low to moderate probability) of realizing the payoff. These findings are consistent with results from a recent study by Kluger and colleagues (2004), who relate RF to the varying probabilities that an event or phenomenon will occur. These authors find that when people consider low to moderate probabilities (i.e., around 30\%), both promotion- and preventionfocused respondents have the same subjective interpretation of the likelihood of the phenomenon. In contrast, moderate to high probabilities (i.e., equal or greater than $80 \%$ ) cause promotion-focused people to overestimate the probabilities, relative to prevention-focused people.

In our second study, we replicate the findings from Study 1 with regard to RF (i.e., reward type) and extend the design by incorporating alternative ways of presenting information about the payoff probability. The results show that promotion-focused retailers, relative to prevention-focused retailers, perceive the reward as more valuable and are more likely to 
invest when the payoff information is presented verbally. The reverse is true for preventionfocused retailers, who seem to prefer numerical presentation. This finding is robust across two types of payoff (i.e., low versus high) and thus supports previous research by Semin, Higgins, and Montes (2005), which suggests that abstract information aligns better with a promotion focus. The underlying explanation for this fit notes that a promotion focus relates to ideals (i.e., hopes and aspirations), whereas concrete information is more congruent with prevention focus associated with ought goals (i.e., security and protection). Because verbal expressions better convey abstract information, promotion-focused retailers experience better fit when they process verbal information. Abstract language best captures eagerness strategies in goal pursuit (e.g., attracting new customers), which by their nature are broad and general (Semin, Higgins, and Montes 2005). In contrast, numerical expressions convey more concrete information, so prevention-focused retailers experience better fit when processing numerical information that captures vigilance strategies, characterized by a focus on details and concrete information (Semin, Higgins, and Montes 2005). Our finding extends $\mathrm{RF}$ research by showing that the presentation format of information makes a substantive difference. In addition, we find a significant influence of value perceptions and investment intentions on loyalty, which corroborates prior loyalty program research (Yi and Jeon 2003).

Finally, our research contributes to RF literature by assessing the impact of trait RF in situations in which we experimentally manipulate RF. Our results refute the assumption, frequently made in RF theory, that state RF dominates trait RF (Higgins and Spiegel 2004) to the extent that it nullifies the effect of trait RF on attitude and behavioral intentions. Across both experiments, we demonstrate the significant influence of trait RF on the outcome measures, even in the presence of primed RF. Therefore, trait RF may explain additional variance in the outcome variables.

\section{Limitations and Future Research Directions}

Every study suffers from limitations, and this research is no exception. A promotion focus considers limitations as opportunities for developing a research agenda that attempts to gain a more in-depth understanding of retailers' responses to CRPs. First, research on risk and uncertainty (see $\mathrm{Wu}$, Zhang and Gonzales 2007) typically exposes respondents to multiple options and multiple payoffs (e.g., choose option A and get payoff $\mathrm{X}$ versus choose option $\mathrm{B}$ and get payoff $\mathrm{Y}$ ) to assess complementarity. In reference to our CRP context, further research should examine multidimensional manipulations (e.g., payoff obtained versus payoff forgone).

Second, in our experimental design, we use different types of rewards to represent promotion and prevention foci. This approach strengthens the priming effect through association with the description or even the name of the program, such that direct mail is associated with promotion. Alternatively, stimuli could be framed in neutral terms, coupled with either promotion or prevention information, which may reflect situationally induced foci 
better - the same glass may be perceived as either half full or half empty (Higgins 2002). Additional research should investigate whether alternative ways of manipulating situational RF yield equivalent results.

Third, we do not distinguish between retailers' short- and long-term goal, which represents an important limitation in view of the so-called temporal component of RF (Pennington and Roese 2003). According to RF theorists, long-term goals may be more aligned with a promotion focus, whereas short-term goals may reflect a balanced consideration of both promotion and prevention concerns (Pennington and Roese 2003). Therefore, this temporal dimension may account for some variance in the reward investment decisions and program loyalty. Further research should take this important nuance into account.

Fourth, we measure the construct relationships at one point of time and thus fail to consider that retailers operate in very dynamic environments, which may have an impact on CRP investment decisions. For example, the timing of reward presentation may help determine the retailers' response, especially with regard to the point in the fiscal year. A longitudinal approach could integrate the impact of these factors.

Fifth, we do not consider the type of CRP setting or the cultural background of the research setting. This important limitation ignores that CRPs frequently are applied in an international context, partitioned by market segments rather than national cultures. For example, Western cultures are more promotion prone, whereas people in Asian countries tend to be more prevention oriented (Aaker and Lee 2001). Further research is needed to test empirically whether the findings of this study generalize across different cultures.

\section{Managerial Implications}

Finally, our findings suggest several managerial implications. First, the type of reward and its expected payoff jointly determine investment decisions and retailer value perceptions, which implies that the administration of CRPs should focus on a careful and deliberate positioning of the program rewards. If retailers perceive that a reward offers a low monetary payoff, it matters little how the reward gets communicated, because either a promotion or a prevention focus will lead to the same point redemption likelihood. Because CRPs usually include a range of rewards; expected payoffs likely vary. Our results suggest that if the payoff is perceived as high, it is more effective to position the reward with a promotion focus. That is, when managers want to offer a high payoff reward, they should communicate it using a promotion frame to enhance its perceived value. For example, by pointing out that the successful completion of a direct mail program would mean reaching more potential customers and yield a very attractive ROI, managers can appeal more strongly to promotion-focused retailers.

Second, managers should pay careful attention to the mode used to communicate CRP rewards. Our results clearly demonstrate that the best presentation of the payoff for promotion-focused rewards uses words, whereas the best presentation of the payoff for 
prevention-focused rewards employs numbers. The strong influence of the payoff, mode of communication, and type of reward on value perceptions and investment decisions further suggests that managers must take these reward attributes into account when designing, targeting, and promoting CRPs to members.

Third, program members' regulatory disposition also influences investment intentions. Therefore, including items to measure a retailer's regulatory orientation, perhaps as part of an annual satisfaction or new CRP member survey, could help managers develop insights into retailers' attitudes and behavior toward point investments. Alternatively, they could assess a retailer's regulatory orientation more unobtrusively by analyzing written documents (e.g., marketing plans, annual reports) and purchase patterns. If an annual report contains an objective such as "maintaining customer satisfaction level to secure our position in the marketplace," the retailer likely has a prevention focus, whereas a greater focus on growth, market expansion, and innovation implies a promotional focus. Overall, we conclude that matching the format of the payoff presentation with aspects of RF can enhance the effectiveness of CRPs. 


\section{CHAPTER 5}

\section{Getting a Discount or Sharing the Cost? \\ The Influence of Regulatory Focus Fit on Consumer Responses to Private Health Insurance Policies}

This research examines the regulatory focus fit effect on perceived value and purchase intentions in the context of private health insurance decisions. The so-called value-from-fit effect is assessed in conjunction with the effect of risk perceptions. Value-from-fit results from the interaction effect between regulatory focus concerns (discount vs. cost-sharing policies) and message framing (gain vs. loss). The results show that the fit effect on purchase intentions is fully mediated by the perceived value of a health insurance policy, and perceived value functions as partial mediator of the effect of risk perceptions. The relative strength of the regulatory fit effect in explaining customer perceived value is small compared with the impact of perceived risk. Nevertheless, this study advances some importance implications of the fit effect for the design and positioning of health insurance policies. 


\section{Introduction}

As a result of increasing competition in health insurance markets, insurance companies face significant challenges (Chakraborty, Ettenson, \& Gaeth, 1994; Maarse \& Bartholomee, 2007). Among lower-income U.S. consumers, for instance, rising health insurance costs have significantly decreased disposable income, so a substantial segment of consumers either underpurchases or does not purchase coverage for health care costs at all (Lueck, 2005). At the same time, consumers pay more than they should, beyond the actuarially fair value, for some types of health insurance (Johnson, Hershey, Meszaros, \& Kunreuther, 1993; Pauly, Herring, \& Song, 2006). Some observers thus conclude that present-day health care is suffering from an insurance crisis (Chavez, 2007). Reports also indicate annual customer turnover reaches as high as $20 \%$ in some European countries (Greß, 2006; Maarse \& Bartholomee, 2007). In making insurance purchase decisions, consumers apparently perceive little variation among the range of services offered by health insurers (Custers, Arah, \& Klazinga, 2007; Gabel, 2004). In combination, these issues emphasize the need to adopt a strategic focus toward marketing health insurance services that is based on a thorough understanding of the factors that underlie consumer decision-making processes and consequent behavior.

Technically, health insurance is a risk-sharing mechanism that transfers the risk of paying for medical care from an insured to an insurer by pooling medical payment across consumers and time (Rejda, 1998). To avoid larger health and financial risks, individual consumers may choose the levels of coverage they wish to obtain. Thus, information about risk in health insurance policies is important for insurance decisions. However, previous research on health insurance purchase decisions (e.g., Johnson et al., 1993; Schoemaker \& Kunreuther, 1979) reveals that people have limited abilities to process information about insurance policies in general and risk in particular. These limits often result in a systematic bias in consumer decision making (e.g., consumers perceive policies with rebates as more attractive than those offering deductibles). Furthermore, decision making may be affected substantively by the framing of coverage and benefits (Johnson et al., 1993). Yet thus far, no study systematically assesses which mechanisms produce risk-distorted results. Furthermore, it remains unclear how consumer beliefs and abilities, in conjunction with framing, affect consumer perceived value and subsequent behavioral intentions with regard to health insurance purchase decisions.

In this research, we employ regulatory focus theory as a theoretical basis to explain consumers' attitudes and behavioral intentions toward individual health insurance policies. Building on the hedonic principle of people's motivation to approach pleasure and avoid pain, regulatory focus theory posits that two types of foci exist: a promotion focus, which aims to achieve positive outcomes, and a prevention focus, which attempts to avoid negative outcomes (Higgins, 1997). Accordingly, people evaluate a decision more favorably when 
they experience a fit between their own foci and the framed information (Avnet \& Higgins, 2006). This result, labeled the value-from-fit effect (Higgins, 2002), is relevant for examining health insurance purchase decisions, which are influenced by both individual characteristics and the presentation of information about insurance policies (Schoemaker \& Kunreuther, 1979). Moreover, fit effects produce a positive feeling that reflects the unconscious affective processing of information during an object evaluation (i.e., feelings as information, Cesario, Grant, \& Higgins, 2004; Higgins, Idson, \& Freitas, 2003; Schwarz \& Clore, 1983). Thus, the main objective of this study is to assess how regulatory fit might be used to explain consumers' decisions in the context of health insurance purchasing. Specifically, we address three critical issues.

First, consumers' insurance decisions involve a trade-off among the risks associated with their health conditions, financial security, and the benefits of an insurance policy (Pauly, 2007). A determinant factor that guides consumers' choice is personal excess, or the combination of the premium and personal contributions (Chakraborty et al., 1994). Such combinations involve a discount (i.e., emphasizing a promotion focus) or cost-sharing (i.e., emphasizing a prevention focus) focus, and diversity in framing occurs (e.g., loss- or gainframed messages); therefore, regulatory focus theory predicts that consumer responses to a health insurance policy result from the regulatory focus and the alternative framing schemes (Lee \& Aaker, 2004). This study attempts to theoretically support and empirically verify this prediction and establish regulatory fit as an explanatory mechanism.

Second, previous research uses a variety of dependent variables, ranging from attitudinal (e.g., brand attitude) to behavioral (purchase decisions) constructs, as outcomes of value-from-fit (Higgins, 2002). Across this range of outcome variables, considerable variation occurs in the magnitude of the value-from-fit effect (cf. Higgins, $2003 \mathrm{cf}$. Cesario et al., 2004). Yet no research examines whether these divergent results are due to differences in the predictor-criterion relationship (i.e., the impact of value-from-fit on attitude vs. behavior). We therefore propose that value-from-fit represents an important predictor of perceived value as a criterion variable and that examining this relationship can further our hitherto limited understanding of value creation in the field of health insurance. Specifically, we investigate whether the perceived value of a health insurance policy is a full or partial mediator of the value-from-fit effect for insurance purchase decisions. In this sense, we contribute to regulatory fit theory and shed further light on the nature of predictor-criterion relationships.

Third, recent research shows that consumer attitudes toward financial risk influence choices of the level of deductibles and premiums in other types of insurance (e.g., automobile) (Cohen \& Einav, 2007). However, particularly with respect to health insurance, a risk attitude may be linked to the health status of consumers (Cardon \& Igal, 2001). To date, perceived financial and health status have served as focal constructs that influence customer decisions about health insurance. We also incorporate perceived financial risk and perceived health risk as context-specific determinants of variance in consumer perceived value. Thus, 
we distinguish carefully between risk perceptions as a result of deliberate cognitive processing and those derived from the value-from-fit effect, produced as a result of unconscious affective processing, as determinant factors of perceived value. This distinction enables us to determine the relative impact of value-from-fit that results from the specific presentation of a health insurance policy.

\section{Theoretical Framework and Research Hypotheses}

According to regulatory focus theory, promotion and prevention foci are two distinct selfregulation strategies that not only reflect a person's predispositional characteristics but also can be momentarily induced by a priming task or situation (i.e., state variable) (Crowe \& Higgins, 1997). Therefore, the notion of regulatory fit suggests people will engage either more strongly or weakly in a particular activity when the manner to engage in the activity sustains or does not sustain their regulatory orientation. In the context of persuasion, research on regulatory fit shows that the fit effect influences the perceived value of an object under evaluation (Lee \& Aaker. 2004), enhances motivational intensity in goal pursuit (Avnet \& Higgins, 2006), and affects how people feel about their decisions (Higgins, 2002).

Three explanations appear in the literature to account for the impact of the fit effect on perceptions of value. First, a relatively high degree of fit leads to more fluent processing of information, resulting in more favorable evaluations (i.e., processing fluency account). For example, Lee and Aaker (2004, p. 207) note that "promotion concerns highlighted in a gain frame and prevention concerns in a loss frame fit with how people naturally think about the issues. Hence, the ideas conveyed in the messages are conceptually fluent and are more persuasive." Second, high levels of fit create a sense of "feeling right" (e.g., Higgins et al., 2003), which subsequently transfers to evaluations. Third, Higgins (2006) proposes that the level of engagement in a particular activity (i.e., working on an experimental task, evaluating an object) magnifies the value experience related to that activity, which echoes a previous finding by Forster, Higgins, and Idson (1998) that suggests persistence in the execution of a task is greater for participants who experience fit rather than nonfit.

The principle of regulatory fit seems particularly relevant in the context of customer preference and choice for a private health insurance policy, because the fit effect may explain the nature of the relevant relationship between the perceived value of health insurance and purchase intentions. The experience of a fit effect may be transferred to perceived value and purchase decisions, because it intensifies the aforementioned attitudes and behavior (Higgins, 2005). For example, when consumers possess prior beliefs that the reward has a high value, their experience of the fit effect (i.e., feeling right) should positively influence their purchase intentions. In this case, the process relates to affective processing of information.

We examine in particular whether evaluations of a health insurance policy enhance the perceived value of the health insurance policy and promote purchase decisions. Specifi- 
cally, we consider insurance policies whose presentation focuses on either a promotion concern (i.e., getting a discount) or a prevention concern (cost-sharing) and that communicate these concerns using gain- or loss-framed messages. In subsequent sections, we examine the interaction of choice framing and message framing on the perceived value of an insurance policy. Because the evaluation of a health insurance policy involves risk perceptions, we also discuss the influence of perceived health and financial risks on value perception.

\section{The Interaction of Choice Frame and Message Frame}

Recent studies on regulatory fit reveal that people's regulatory focus (promotion vs. prevention) moderates the effect of message framing (gain vs. loss) on persuasion (Avnet \& Higgins, 2006; Lee \& Aaker, 2004). Lee and Aaker (2004) demonstrate the existence of a fit effect in the context of health-related products (e.g., health drinks, sun lotion) and find that messages framed as a gain are more persuasive when the message is promotion focused, whereas messages presented as losses are more effective when they are prevention focused. For example, an advertisement for a health drink that highlights the need for energy would be more effective if the advocated messages were framed in a gain (i.e., "If you drink this, you will get energized") than a loss (i.e., "If you don't drink this, you will miss out on getting energized") context, whereas an advertisement that highlights concern about preventing clogged arteries would be more persuasive if the advocated messages were framed as a loss (e.g., "If you don't drink this, you will miss out on the prevention of clogged arteries") rather than a gain (i.e., "If you drink this, you can prevent clogged arteries") (Aaker \& Lee, 2004).

A health insurance policy similarly can be framed in terms of promotion concerns (i.e., getting a discount) or prevention concerns (i.e., avoiding cost-sharing), so consistent with regulatory fit theory, we expect that those consumers who evaluate policies with promotion concerns will perceive greater value from the reward if they view gain-framed rather than loss-framed messages. In contrast, consumers evaluating policies with prevention concerns will perceive more value from the policy if it appears in loss-framed rather than gain-framed messages. We therefore hypothesize:

H1. The perceived value of a policy will be greater for consumers evaluating a discountframed policy with a gain-framed message rather than a loss-framed message, whereas the reverse is true for consumers evaluating a cost-sharing-framed policy with a loss-framed message. 


\section{Value Perceptions and Purchase Intentions}

A fundamental assumption in consumer decision theory states that consumers choose an option (e.g., insurance policy) that reflects their preferences (Simonson, 2005). We expect the same assumption to hold in our study, because types of health insurance policies have high personal relevance for customers. Thus, when evaluating a policy with deductibles and premiums, those who perceive that the policy has a high value should be more likely to purchase health insurance.

H2. The perceived value of a policy relates positively to purchase intentions.

Extant empirical research indicates that the fit effect drives attitudes and behaviors (e.g., Cesario et al., 2004; Higgins et al., 2003). However, these studies also reveal that the magnitude of the fit effect varies considerably. For instance, Higgins et al. (2003) show that the strength of the fit effect could be as high as $40-60 \%$, but Cesario et al (2004) find relatively low strength of only around $12 \%$. In addition, studies that use behavioral intention measures to tap the fit effect fail to examine how value transfers from the perceived value of an object to behavioral intentions. In Cesario et al.'s (2004) consumption studies, the effect of regulatory fit appears significant for both attitudes toward eating more vegetables and intentions to eat more vegetables, yet these authors do not report a mediation analysis. Divergent results may be obscured by the different nature of the outcome measures. Therefore, we extend previous research by examining specifically whether the fit effect on behavioral intentions is fully or partly mediated by perceived value.

On the basis of attitude theory models (e.g., Ajzen, 1991; Ajzen \& Fishbein, 1980; Eagly \& Chaiken, 1993), we propose that perceived value fully mediates the effect of value-from-fit on purchase intentions. We base our prediction on previous attitude research that empirically shows perceived value is an attitudinal construct that often exerts a strong influence on behavioral intentions (e.g., Theory of Reasoned Action, Ajzen \& Fishbein, 1980; Theory of Planned Behavior, Ajzen, 1991; composite model of attitude, Eagly \& Chaiken, 1993). Although the perceived value of an insurance policy refers to the consumer's evaluation, the purchase intention reflects his or her preference for a specific behavior. Theory pertaining to attitude formation posits that attitudes drive behavioral preference, and previous regulatory focus research offers some evidence for such attitude-based preferences. In Higgins et al.'s (2003) study of the valuation of coffee mugs, the fit effect increases the value of the mug, which in turn affects participants' choices. In this example, preference is due to the increased value perception. Thus, we expect that the fit effect transfers directly to perceived value, which then affects behavioral intentions. Therefore, we hypothesize:

H3. Perceived value mediates the impact of the fit effect on purchase intentions. 


\section{Risk Perceptions}

Extant research into health insurance reveals that consumers' decision to purchase certainly types of health insurance policies depends largely on their perceptions of their health status and risks associated with financial loss (e.g., Bakker, van Vliet, \& Van de Ven, 2000; Pauly $\&$ Herring 2007). We thus develop hypotheses regarding the impact of perceived health and financial risks on consumer purchase decisions for health insurance. The risk terms we use in this section refer to risks as domain-specific constructs, that is, as drivers or key explanations of insurance purchase intentions, whereas risks in message framing refer to the risks of buying a specific policy scheme that produces a gain (i.e., getting a discount) or loss (i.e., cost-sharing).

\section{Perceived Health Risk}

With respect to the impact of perceived health risks on consumer health insurance decisions, previous research indicates that healthy people likely opt for a policy with a high deductible (i.e., the amount paid by an insured each year before the insurer start paying health care costs) and low monthly premiums (Pauly \& Herring, 2007). Conversely, unhealthy people opt for a low or even no deductible (i.e., full coverage) and, as a consequence, pay high monthly premiums. Given these findings, we propose:

H4. Perceived health risk relates negatively to (a) the perceived value and (b) the purchase of a health insurance policy with a high deductible.

\section{Perceived Financial Risk}

Choosing a health insurance policy also involves financial risk-taking, because it determines the out-of-pocket expenditures that an insured must pay in case he or she incurs health care costs. One of the essential elements of health insurance, risk transfer (Rejda 1998), means that the health insurer, which has a stronger financial position from which to pay for the loss (e.g., treatment cost) than the insured, takes on the financial risks, and the insured only pays a fixed cost of the premium. If a consumer decides to purchase health insurance, he or she lowers personal financial risk, because all medical expenses will be paid by an insurer. However, without insurance coverage, the consumer faces greater financial risk because he or she must bear all health-related costs. A recent study of the relationship between saving and insurance coverage (Guagrilia \& Rossi, 2004) reveals that, all else being equal, people who perceive that their financial situation is bad will opt for a health insurance policy with higher deductibles, because they only need to pay low monthly premiums. Marquis et al. (2006) also find that a $4 \%$ decrease in the nominal premiums allows healthy customers to switch to a health insurance policy with a $50 \%$ higher deductible. Hence, we propose: 
H5. Perceived financial risk relates positively to (a) the perceived value and (b) the purchase of a health insurance policy with a high deductible.

We present our conceptual framework in Figure 5.1.

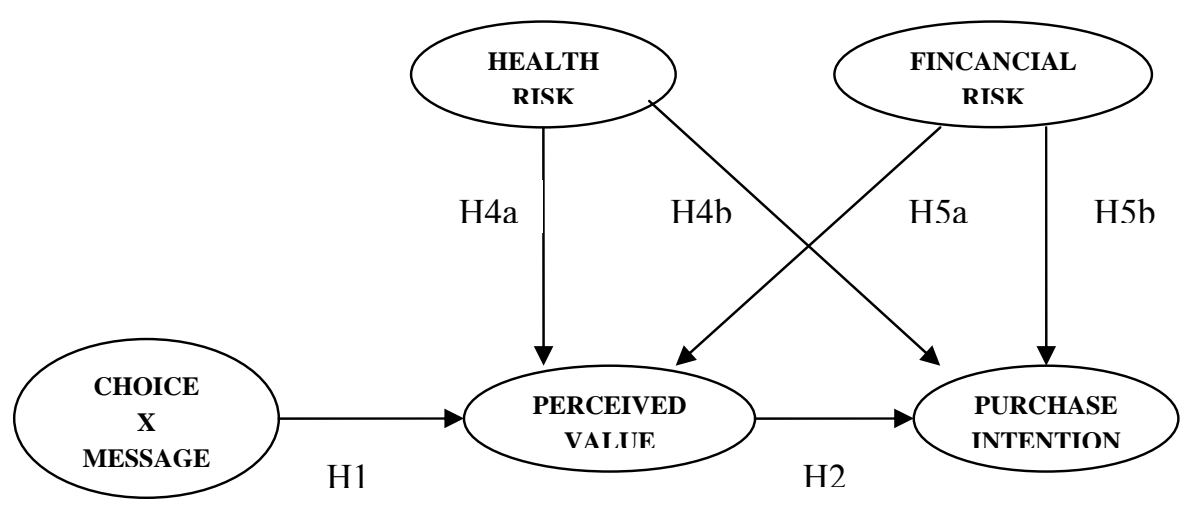

Figure 5.1: Conceptual Model

\section{The Relative Impact of Fit Effect vs. Risk Perceptions}

As explained previously, the fit effect offers additional value, independent of the value derived from the policy. Typically, prior research assesses the strength of fit by comparing response measures (e.g., perceived value of an object) provided by participants in a fit condition with those in a no-fit condition. However, these results obscure the strength of fit relative to the effect of the constructs that are most influential in affecting the consumer. We instead assess the strength of the fit effect by comparing the relative impact of the fit versus domain-related constructs, such as risk covariates (perceived health and financial risks). Because risk perceptions are essential constructs for explaining the perceived value of a health insurance policy and because regulatory fit seems to offer additional value, independent of the value derived from the risk covariates, we propose the following hypothesis:

H6. The fit effect, relative to the effect of perceived health risk and perceived financial risk, explains additional variance in the perceived value of a health insurance policy. 


\section{Empirical Study}

\section{Research Setting}

We conducted our study in the Netherlands, a country with a new health regulation system (January 2006). The guiding principle behind the new program was to offer accessible and affordable private health insurance to the entire population (Maarse \& Bartholomee, 2007). Under the new system, every resident in the Netherlands is obliged to take out a basic health insurance scheme from an insurance company of his or her choice. Insurance companies were obliged to accept all applicants, regardless of their health status (within a limited timeframe).

To test our hypotheses, we design a $2 \times 2$ between-subjects experiment, in which we manipulate the type of choice framing (discount vs. paying a penalty), as well as type of message framing (gain vs. loss). We assign respondents randomly to one of four scenarios and collect data through a questionnaire that contains the general instructions, scenario descriptions, and a list of items to measure value perceptions, purchase intentions, switching intention commitment, perceived health risk, and perceived financial risk. In addition, demographic measures gather age and gender variables.

\section{Procedure and Scenarios}

The scenarios ask respondents to imagine that they have owned a health insurance policy that requires them to pay a monthly premium of $€ 87.50$ with no deductibles. They subsequently received a new opportunity to switch to a new health insurance policy offered by another insurer, for which they would have to pay a certain monthly premium, with or without deductibles, such that the combination of the premium and deductibles is positioned as either gaining a discount or paying a penalty. This situation and the combinations of premiums and deductibles we use appear in Table 5.1.

Table 5.1: Combinations of Premiums and Deductible Used in the Study

\section{Policy Scheme}

Present $€ 87.50$ with no deductible

Alternative

A. $€ 87.50$ with no deductible

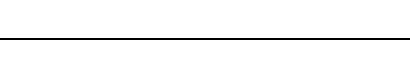

C. $€ 72.50$ with a deductible of $€ 500$ $\underline{\text { Discount condition }}$

or B. $€ 87.50$ with a deductible of $€ 500$, plus a discount of $€ 15$ in the monthly premium

Cost-sharing condition

or D. $€ 72.50$ with no deductible, plus a cost-sharing of $€ 15$ in the monthly premium 
Notes: Option A is essentially the same as the present option and option D, whereas option B is the same as option $\mathrm{C}$. The study advocates purchasing option $\mathrm{B}$ in the discount condition and option $\mathrm{C}$ in the costsharing condition.

The amount of the deductible serves to position each scheme as either offering a discount or inducing a penalty of $€ 500$, which is the maximum deductible that applicants can choose under the new health care regulation system. After reading the description, respondents considered a message framed as either a gain or a loss, aimed at persuading them to switch to the offered alternative. We include a sample scenario as an illustration of the manipulations used in Appendix C.1 and C.2.

\section{Construct Development}

In addition to the experimental factors, we include five constructs in our model: value perception, buying intention, switching intention commitment, perceived health risk, and perceived financial risk. We specify a second-order construct of purchase intention, in which buying intention and switching intention commitment represent its first-order constructs. In turn, we measure value perceptions, buying intentions, switching intention commitment, and perceived financial risk with seven-point Likert scales, anchored by strongly disagree [1] and strongly agree [7]. To measure perceived health risk, we employ a five-point semantic scale ranging from poor [1] to excellent [5]. All constructs consist of multiple items, adapted to the characteristics of the research setting and the scenarios. The three items used to measure value perception derive from previous research by Yi and Jeon (2003). We develop a three-item measure of buying intention and employ another three-item measure for perceived financial risk, based on previous research by Pennings and Smidts (2000). To measure switching intention commitment, we use four items adapted from Johnson, Hermann, and Huber (2006) and Fullerton (2005). All the measurement items appear in Table 2. We also include items in the questionnaire that serve as manipulation checks for the type of choice frame and regulatory focus. The discount-framed scenarios include the item, "My concern in choosing the insurance policy is to get the discount," whereas the penalty-framed scenarios feature, "My concern in choosing the insurance policy is to avoid the penalty." Both items rely on seven-point Likert scales, ranging from strongly disagree [1] to strongly agree [7]. For these manipulation checks, we use a test value of 4; a value greater than the scale midpoint implies that the manipulation operated successfully.

\section{Data Collection}

Of the questionnaires sent to customers of a large health insurance company, we obtain a net sample of 193 (response rate: 65\%). The following demographic profile emerges from the sample: $52 \%$ of the respondents are men, and $24 \%$ are aged between 26 and 35 years, $19.3 \%$ 
between 36 and 45, 18\% between 46 and 55, and 5\% are older than 56 years. We compare this sampling with the customer profile of the company database and find no significant difference with regard to these demographic factors.

\section{Data Analysis}

To test the hypotheses, we employ partial least squares (PLS) path modeling; specifically, we use PLS-Graph software version 3.0 (Chin, 2001) to obtain parameter estimates for the measurement and structural models. The PLS method has several advantages over a covariance-based analysis or LISREL, such as the ability to handle non-multivariate normal data, multicollinearity among independent variables, small samples, and formative indicators (Chin, 2001; Tenenhaus, Vinzi, Chateline, \& Lauro, 2005). In addition, PLS is suitable for modeling factorial designs. Compared with traditional techniques of experimental designs (e.g., (M)AN(C)OVA), PLS has several clear advantages, including the ability to control for measurement error, incorporate covariates in the model, and offer greater modeling flexibility (Mackenzie, 2001).

To model the experimental data with a structural equation framework, we specify single indicators for the manipulated factors, namely, types of choice frame and type of message frame, as well as the interaction effect. These manipulations employ effect coding, such that discount is coded as 1 and penalty as -1 , and gain-framed messages are coded as 1 and loss-framed messages as -1 . Effect coding is preferable to dummy coding $(0,1)$, because it produces tests of main effects and interactions for any models involving interaction terms, whereas the latter does not (O’Grady \& Medoff, 1988; Pedhazur, 1997).

The interaction frame entails the product term of the two effect-coded variables (choice frame $\times$ message frame). The dependent variables, value perceptions, buying intentions, switching intention commitment, perceived health risk, and perceived financial risk, represent reflective indicators. As mentioned previously, we specify purchase intention as a formative second-order construct comprised of the first-order buying intentions and switching intention commitment factors.

We examine the reliability, convergent validity, and discriminant validity of these indicators and, in Table 5.2 provide the PLS parameter estimates for the measurement model. Table 5.3 details the measures for assessing the internal consistency of a given block of indicators according to composite reliability (Fornell \& Larcker, 1981) and average variance extracted (AVE; Fornell \& Larcker, 1981). The composite reliability scores for all constructs with reflective indicators exceed the cut-off value of .7 proposed by Nunnally and Bernstein (1994). The AVE for all constructs exceeds the cut-off value of .5 suggested by Fornell and Larcker (1981). To assess the discriminant validity of each construct, we rely on three methods. First, a construct should share more variance with its measures than it shares with other constructs (Chin, 1998; Hulland, 1999), and therefore, the square root of the AVE should exceed the intercorrelations of the construct with other constructs. In our 
study, as we display in Table 3, the square root of the AVE for all constructs is greater than all corresponding correlations (Fornell \& Larcker, 1981). Second, cross-loadings with constructs other than that hypothesized are not substantial in magnitude (<.3; Chin, 1998; Hulland, 1999). Third, the correlations of the residual terms across blocks do not exceed |.2| (Falk \& Miller 1992; Fornell \& Bookstein 1982). Therefore, we conclude that all constructs exhibit satisfactory discriminant validity. We next assess the convergent validity of the constructs by inspecting their factor loadings. As we show in Table 2, the factor loadings for all constructs with reflective indicators are greater than .50 , which demonstrates acceptable convergent validity. The loadings of the first-order factors, buying intention and switching intention commitment, on the second-order factor of purchase intention exceed .8 (i.e., .868 for buying intention and .827 for switching intention commitment).

Because our data feature self-reported measures from the same persons in the same measurement context, we consider the potential for common method variance (Podsakoff et al., 2003). We check for common method variance by means of Harman's single-factor test and conduct an exploratory factor analysis to extract the unrotated factor solution by including all items in the analysis. Common method variance exists if a single factor accounts for the majority of the covariance. The results indicate that no single factor with an eigenvalue greater than 1 accounts for more than $37 \%$ of the variance; therefore, common method variance is not a problem for these data.

\section{Results}

\section{Manipulation check}

Our manipulation checks indicate that both choice frames differ significantly from the scale midpoint (discount-framed choice: $\mathrm{t}(94)=8.093, p<.001$; cost-sharing framed choice: $\mathrm{t}(97)$ $=8.806, p<.001)$. The regulatory focus manipulation thus works as intended.

\section{Hypotheses testing}

To estimate the path coefficient estimates of the structural model, we run a nonparametric bootstrapping with 500 resamples and provide the results in Table 5.3. None of the main effects is significant, and we find a positive significant interaction effect on value perception $(\beta=.152, p=.003)$, in support of H1 (Figure 5.2). 
Table 5.2: Measurement Instruments (Survey)

\begin{tabular}{|c|c|}
\hline Items & $\begin{array}{l}\text { Standardized } \\
\text { Loading }\end{array}$ \\
\hline \multicolumn{2}{|l|}{ Perceived Financial Risk } \\
\hline I am willing to take financial risks in order to realize savings & 0.903 \\
\hline I like taking financial risks & 0.697 \\
\hline $\begin{array}{l}\text { I am willing to take financial risks when choosing personal excess, in order to realize } \\
\text { savings }\end{array}$ & 0.933 \\
\hline \multicolumn{2}{|l|}{ Perceived Health Risk } \\
\hline How do you rate your health condition at the moment? (very poor-excellent) & 0.903 \\
\hline In the near future, how do you rate your health condition (very poor-excellent) & 0.648 \\
\hline \multicolumn{2}{|l|}{ Value Perception } \\
\hline The proposed health insurance scheme is a great value & 0.849 \\
\hline The proposed health insurance scheme is attractive & 0.895 \\
\hline The proposed health insurance is what I have wanted & 0.915 \\
\hline \multicolumn{2}{|l|}{ Buying Intention } \\
\hline It is most unlikely that I will choose this health insurance scheme (reverse coded) & 0.881 \\
\hline I will most likely choose this health insurance scheme & 0.946 \\
\hline My intention to choose this health insurance scheme is high & 0.851 \\
\hline \multicolumn{2}{|l|}{ Switching Intention Commitment (reverse coded) } \\
\hline I want to continue my relationship with my current insurance company & 0.864 \\
\hline My current insurance company has a great deal of personal meaning to me & 0.694 \\
\hline $\begin{array}{l}\text { It would be very hard for me to switch away from my current insurance company right now } \\
\text { even if I want to }\end{array}$ & 0.702 \\
\hline It would be costly to switch from my current insurance company right now & 0.825 \\
\hline \multicolumn{2}{|l|}{ Second-Order Construct } \\
\hline \multicolumn{2}{|l|}{ Purchase Intention } \\
\hline Buying intention & 0.868 \\
\hline Switching intention commitment & 0.827 \\
\hline
\end{tabular}


Table 5.3: Composite Reliability and Correlation among Construct Scores (Square Roots Values of AVE in Diagonals) (Survey)

\begin{tabular}{llllcccccc}
\hline Construct & CR & AVE & $\mathbf{1 .}$ & $\mathbf{2 .}$ & $\mathbf{3 .}$ & $\mathbf{4 .}$ & $\mathbf{5 .}$ & $\mathbf{6 .}$ & 7. \\
\hline 1. CHO $\times$ MES & $\mathrm{Na}$ & na & na & & & & & & \\
2. VAL & 0.917 & 0.786 & 0.206 & $\mathbf{0 . 8 8 7}$ & & & & & \\
3. HRISK & 0.759 & 0.677 & 0.108 & -.134 & $\mathbf{0 . 8 2 3}$ & & & & \\
4. FRISK & 0.861 & 0.681 & 0.101 & 0.533 & -0.137 & $\mathbf{0 . 8 2 5}$ & & & \\
5. BUY & 0.923 & 0.799 & 0.162 & 0.595 & -0.184 & 0.383 & $\mathbf{0 . 8 9 3}$ & & \\
6. SWITCH & 0.856 & 0.600 & 0.124 & 0.388 & -0.303 & 0.318 & 0.415 & $\mathbf{0 . 7 7 5}$ & \\
Second-order construct & & & & & & & & \\
7. PI & 0.865 & 0.588 & 0.172 & 0.600 & -0.278 & 0.421 & 0.889 & 0.785 & $\mathbf{0 . 7 4 7}$ \\
\hline
\end{tabular}

Notes: $\mathrm{CON}=$ type of choice frame; MES = type of message frame; $\mathrm{VAL}=$ perceived value; $\mathrm{BUY}=$ buying intention; SWITCH = switching intention; HRISK = perceived health risk; FRISK = perceived financial risk, $\mathrm{PI}=$ purchase intention, $\mathrm{CR}=$ composite reliability; and $\mathrm{AVE}=$ average variance extracted.

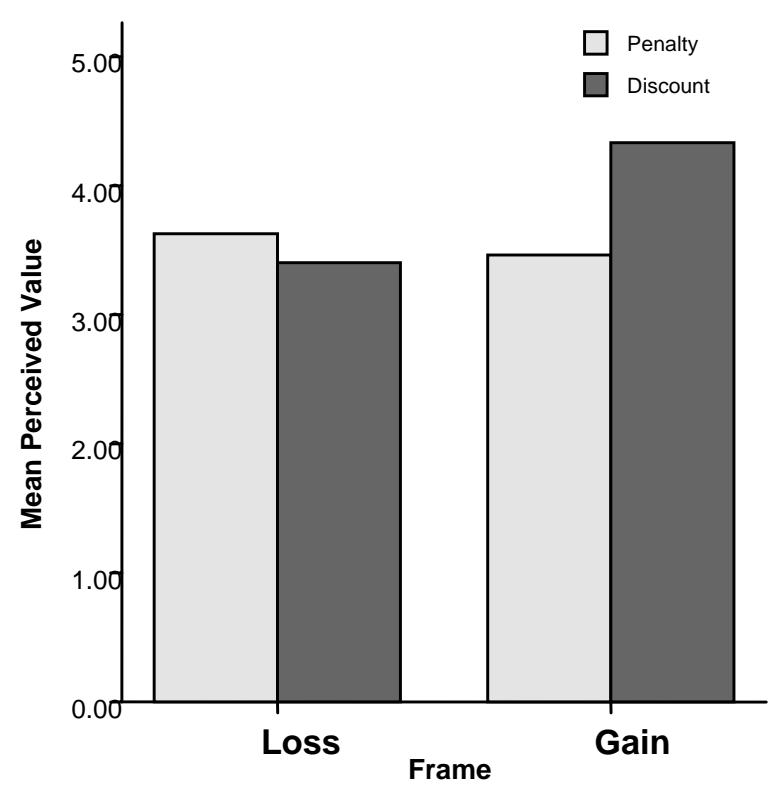

Figure 5.2 Interaction between Choice Frame and Message Frame on Perceived Value

In the light of this significant interaction effect, we conduct two simple effect tests to determine the nature of the interaction effect (Keppel \& Wickens, 2004; Pedhazur, 1997). To examine the differences between gain and loss-framed message separately for discount and penalty, we test (1) the mean differences of gain-framed and loss-framed with a discount and (2) the mean differences of gain-framed and loss-framed messages with a penalty. Thus, we split the data according to the choice frame category, using effect coding, namely, gain frame as 1 and loss frame as -1 . Next, we regress the value perception on the message frame type for each type of choice frame. Again, we use PLS Graph with nonparametric boot- 
strapping with 500 replications to obtain the parameter estimates of the relationship between the type of message frame and value. In the discount condition $(n=95)$, we obtain a positive significant relationship between message frame type and value $(\beta=.134, p=.004)$; that is, with a discount-framed scheme, value perceptions are greater for the gain frame than for the loss frame. In the penalty condition $(n=98)$, we obtain a negative marginally significant relationship between type of message frame and value perception $(\beta=-.105, p=.06)$, which indicates that in a penalty-framed scenario, value perceptions are greater for the loss frame than the gain frame. Altogether, these results support H1.

We find a significant positive relationship between value perceptions and purchase intentions $(\beta=.606, p<.001)$, in support of $\mathrm{H} 2$. With respect to the impact of covariates on value perceptions and purchase intentions, the results indicate a significant relationship between perceived health risk and value perceptions $(\beta=-.097, p=.05)$, in support of $\mathrm{H} 4 \mathrm{a}$, and a negative relationship between perceived health risk and purchase intentions $(\beta=-$ $.191, p=.003$ ), in support of H4b. Finally, we uncover a positive relationship between perceived financial risks and value perceptions $(\beta=.520, p<.001)$, in support of H5a, and between perceived financial risks and purchase intentions $(\beta=.148, p<.05)$, in support of H5b.

Table 5.3: Results for Structural Model

\begin{tabular}{llccl}
\hline Hypothesis & Relationship & Coefficient & $\boldsymbol{p}$-Values & Result \\
\cline { 1 - 3 } H1 & CHO x MES $\rightarrow$ VAL & 0.152 & 0.003 & Supported \\
H2 & VAL $\rightarrow$ PI & 0.495 & $<0.001$ & Supported \\
H4a & HRISK $\rightarrow$ VAL & -0.097 & 0.05 & Supported \\
H4b & HRISK $\rightarrow$ PI & -0.191 & 0.003 & Supported \\
H5a & FRISK $\rightarrow$ VAL & 0.520 & $<0.001$ & Supported \\
H5b & FRISK $\rightarrow$ PI & 0.148 & 0.02 & Supported \\
\hline
\end{tabular}

Notes: $\mathrm{R}^{2}: \mathrm{VAL}=.347 ; \mathrm{PI}=.420$.

\section{Mediation Analysis of the Fit Effect}

For testing and determining the support for our claim that perceived value mediates the influence of the fit effect on purchase intention, we conduct a mediational analysis with risk covariates that were omitted from the model. Thus, we ensure that the fit effect on perceived value is not contaminated by the impact of risk covariates. We also do not include the main effects from the model, because they are not significant in the prior analysis. Following Baron and Kenny (1986), we conduct three PLS analyses. First, we delete purchase intention from the original model and draw a path from choice $\times$ frame $\rightarrow$ perceived value. The interaction effect on perceived value is significant $(\beta=.224, p<.000)$. Second, we delete perceived value from the original model and draw a path from choice $\times$ frame $\rightarrow$ purchase intention; again, the interaction effect on purchase intention is significant $(\beta=.170, p<$ $.000)$. Third, we include perceived value and purchase intention in the model and draw paths from choice $\times$ frame $\rightarrow$ perceived value $\rightarrow$ purchase intention and from choice $\times$ frame $\rightarrow$ 
purchase intention. The results show that the interaction effect is significant for perceived value $(\beta=.206, p<.000)$, perceived value is significant for purchase intentions $(\beta=.597, p$ $<.000)$, yet the interaction effect is not significant for purchase intentions $(\beta=.048, p>.5)$. Because these results fulfill the necessary conditions to establish mediation (Baron \& Kenny 1986), we obtain support for the mediational role of perceived value, in support of H3.

\section{Testing the Proportion of Variance Accounted for by the Interaction Effect}

As we note in the previous section, perceived health and financial risks are essential constructs for explaining the variance in consumer preference and the choice of insurance policy. In this section, we assess the strength of the fit effect by comparing the relative impact of fit versus the risk covariates. First, we examine a decrement in the proportion of variance accounted for by the interaction effect and the two covariates (Pedhazur, 1997) from the variance explained by the full model in Figure 1. Second, we run two restricted models, nested in the full model, and compute the $\mathrm{R}^{2}$ associated with the value perception for each. The two restricted models include (1) the perceived health risk and perceived financial risk but exclude the two main and interaction effect (restricted model A) and (2) the two main and interaction effects but exclude the perceived health risk and perceived financial risk. Third, we test the significance of the $\mathrm{R}^{2}$ difference $\left(\mathrm{R}_{\text {full model }}^{2}-\mathrm{R}_{\text {restricted model }}^{2}\right)$ for each restricted model using F-tests.

The results in Table 5.4 demonstrate that the full model, restricted model A, and restricted model B account for approximately $34.7 \%, 32.2 \%$, and $8.2 \%$ of the variance in value perceptions, respectively. The $\mathrm{R}^{2}$ difference test also shows that restricted model A reduces the $\mathrm{R}^{2}$ of the original model by $.025(\mathrm{~F}=2.425, p=.067)$, whereas restricted model $\mathrm{B}$ reduces it by $.265(\mathrm{~F}=38.756, p<.001)$. Thus, the fit effect, relative to the effect of covariates, seems to offer a relatively small variance in value perception, in support of $\mathrm{H} 6$.

Table 5.4: Testing the Proportion of Variance Accounted for by the Interaction Effect

\begin{tabular}{lllll}
\hline *Model & $\mathbf{R}^{2}$ & ${ }^{*}$-Difference & F-Statistics & $p$-Value \\
\hline Full model & 0.347 & na & na & na \\
Restricted model A & 0.322 & 0.025 & 2.425 & 0.067 \\
Restricted model B & 0.082 & 0.265 & 38.756 & $<0.001$ \\
\hline
\end{tabular}

*Model A retains perceived health risk and perceived financial risk but excludes the two main and interaction effects. Model B retains the two main and interaction effects but excludes perceived health risk and perceived financial risk. ${ }^{*} \mathrm{R}_{\text {full model }}^{2}-\mathrm{R}_{\text {restricted model, }}^{2}$ na $=$ not available.

\section{Discussion}

This research investigates consumers' reactions to a health insurance policy, using regulatory focus as a theoretical basis. Specifically, we attempt to examine the interaction effect between the manner in which a policy scheme is positioned and communicated, according 
to gain- or loss-framed information. In addition, our research assesses the role of perceived value as a criterion variable that mediates the impact of value-from-fit on insurance purchase intentions. Finally, we examine the impact of the fit effect relative to the impact of perceived risks on consumer value perceptions.

Our results show that the fit effect, operationalized as the interaction between choice framing and message framing, has a positive influence on value perceptions; we find no main effects. That is, people value a health insurance policy if the information about the policy is compatible with the message frame. A gain-framed positioning is more compelling if the information about the health insurance policy highlights promotion concerns, whereas a loss-framed positioning is more effective when the information about the insurance scheme addresses prevention concerns. This result is consistent with Lee and Aaker's (2004) finding that regulatory focus moderates the effect of framing on persuasion.

In addition to demonstrating the fit effect on value perceptions, we reveal that perceived value fully mediates the impact of the fit effect on purchase intentions. In the presence of a strong influence by a context-specific determinant of perceived value, namely, risk perceptions, fit effects offer an additional explanation of the variance in value perceptions, albeit small. However, though its impact on value perceptions is relatively small, we must consider fit effects for several reasons. First, a fit effect that creates a "feeling right" experience often functions as an affective source of information for judgments (i.e., feelings-asinformation, Higgins et al., 2003) and thus indirectly influences consumer choice. Second, fit effects occur beyond people's awareness (unconscious affective processing), unlike risk perceptions that involve more deliberate, conscious, cognitive processing (e.g., "How healthy I am"), which makes them a complementary means to explain variance. Third, whereas risk perceptions are generic beliefs that are difficult to influence directly, the fit effect is directly associated with the details related to the positioning of important aspects of health insurance, such as the presentation of the policy and these details are far more accessible. Thus, we contribute to regulatory fit literature by demonstrating the relative importance of the regulatory fit effect in comparison with a substantive model that explains perceived value creation.

With respect to the influence of risk perceptions on perceived value and behavioral intentions, we identify partial mediation. Risk perceptions affect behavioral intentions both directly and indirectly through perceived value. The finding of an indirect effect of risk perceptions on behavioral intention through the perceived value of a product corroborates extant risk research that examines the tripartite relationship among risk perceptions, attitude toward a product, and behavioral intentions (e.g., Huang, 2003; Townsend \& Campbell, 2004). 


\section{Limitations and Future Research Directions}

As with any research, the findings of our study should be interpreted in the light of several key limitations. First, we limit our manipulations to a single form of maximum deductible levels (i.e., €500) with certain monthly premiums. Other combinations of deductibles and premiums might produce different results (e.g., Schoemaker \& Kunreuther, 1979). Second, choosing health insurance is often a family rather than an individual decision. Therefore, additional research should examine social and socioeconomic factors that may affect the results. Third, we focus only on the choice of individual health insurance and exclude collective health insurance (e.g., a specific insurance scheme arranged by an insurer for group of employees by an employer). In this case, individual insurance decisions likely are determined largely by group agreement. Further research could investigate the influence of regulatory focus in consumer decisions about the type of collective insurance to select. Fourth, we consider only the effects of premiums and deductibles, though rebates (i.e., the portion of the premiums that can consumers receive back) might represent another important element of an insurance scheme. Unlike deductibles, which represent a loss of current wealth, a rebate is a gain, and prior research already has demonstrated the framing effects that occur in the choice between deductibles and rebates (Johnson et al., 1993). Specifically, Johnson et al. (1993) demonstrate that a policy with rebates is more attractive than one that includes equivalent but less expensive deductibles. Additional research therefore should explore the role of regulatory focus in influencing this type of choice. Fifth, we use a sample that is not representative of Western culture. Because culture influences the regulatory focus of consumers (i.e., Western culture is more associated with a promotion focus, Eastern culture with a prevention focus; Aaker \& Lee, 2001; Briley \& Aaker, 2006), further research is needed to broaden our understanding of the role of other factors, especially culture, in affecting consumer decisions about health insurance.

\section{Managerial Implications}

Our findings suggest several managerial implications. First, the type of choice framing and message framing jointly determine the perceived value of an insurance policy. Therefore, insurers should focus on the deliberate positioning of their health insurance plans. Typically, insurers offer consumers who buy deductibles policies that include a premium discount. Our research suggests that insurers also could include a premium cost-sharing offering if consumers decide to take a no-deductible policy. If insurers decide to offer a premium discount, they should communicate it by emphasizing the benefits consumers attain from buying a deductible policy (i.e., getting the discount). However, if insurers include a premium costsharing policy, they can best communicate it by emphasizing the costs associated with choosing the policy, which should prompt consumers to choose a deductible policy. 
Second, insurers should attend to consumers' perceived health and financial risks, which dominate their insurance choices. Insurance managers could measure risk perceptions in consumer surveys, or, using short questionnaire, they could determine consumers' risk perceptions just before they decide to buy a new policy.

Third, positioning a health insurance policy in terms of discount and cost-sharing concerns and gain and loss frames offers a promising and useful strategy for attracting new customers and retaining existing costumers, which can contribute to insurers' baseline.

Fourth, because we find a mediation effect of perceived value, the positioning of an insurance policy should be based on the principle of value creation. Managers might include the benefits of policies and consumer health plans that address promotion and prevention concerns. Including such benefits in the descriptions of policies would enable insurers to enhance their value creation by targeting heterogeneous segments (e.g., mixed promotionand prevention-focused consumers).

Overall, our research thus demonstrates that the fit effect has a systematic and predictable, albeit small, impact on consumer decision making and is therefore quite important to take into account. In addition to risk perceptions, which are more general and form over a longer period of time, the fit effect relates to the (fine-print) details of an insurance policy that can alter consumer preferences and choices. 


\section{CHAPTER 6}

\section{Conclusion}

\section{Synopsis}

In this dissertation, we focused on regulatory fit as a determinant factor of perceived value that can be subsequently transferred to behaviors of consumers participating in loyalty programs. The overall objective was to examine the impact of regulatory fit on consumer responses to the communication of features of the program, such as rewards and benefits. In order to successfully address this overall objective, four research projects were formulated with their specific goals to fill the gap in regulatory focus research. The objectives of the four research projects were:

- To provide a quantitative synthesis of the research concerning the magnitude of the impact of regulatory fit.

- To empirically test the interaction effect between state regulatory focus and the way the reward is positioned on value perception and consumer subsequent behaviors.

- To assess the relative strength of the trait regulatory focus in studies that manipulates regulatory focus.

- To determine the relative impact of fit effect in comparison to context-specific determinants of variance in value perception.

The four research projects presented in this dissertation can be briefly summarized as follows. In chapter 2 we reviewed the impact of fit effect on consumer behavior in studies that use experimental designs and conducted a meta-analysis study to synthesize and integrate empirical evidence of the fit effect. In chapter 3, we described two empirical studies. In the first study, we examined the fit effect as a determinant of reward perceived value and exercise intention in the setting of a health club loyalty program. In the second study, we tested whether consumer traits specific to the domain of loyalty programs mediate the influence of regulatory foci on reward redemption decisions. In chapter 4 , we studied the impact of fit effect in a B-to-B loyalty program designed by a supplier for its retailers participating in the programs. In this chapter, our focus was to study how consumers evaluate uncertainty information regarding reward payoff. Finally, in chapter 5, we study the impact of the fit effect in the context of insurance purchase by building and testing a fit effect model that incorporates a context-specific dominant of perceived value, namely perceived health and financial risk.

In this concluding chapter, we summarize and integrate the findings that have been discussed in each chapter. We end this chapter and dissertation by providing a number of suggestions for future research. 


\section{An Integrated Perspective}

This dissertation comprises of four independent but interrelated studies that serve as building blocks to answering the research questions. In this section, we discuss several issues that unify these studies and their theoretical and managerial implications.

\section{The Impact of the Fit effect}

With regard to the impact of the fit effect on value creation, this dissertation addressed the following topics. First, we examined how consumers' regulatory foci shaped by priming the concern of the reward, i.e., in terms of promotion or prevention benefits interacts with how the message is communicated, i.e., in terms of gain or loss. Second, we empirically assessed how value created as a result of fit (i.e., value-from-fit) influences reward perceived value. Based on our meta-analysis study presented in chapter 2, the impact of fit effect on consumer decision making varies from moderate to large. This finding empirically substantiates the fact that the fit effect does affect value creation and as such plays an important role as a determinant of consumers' behavioral intentions, motivation, and judgment. In chapter 3 , we examined the influence of the fit effect on the perceived value of a reward in health loyalty programs and consumer behavioral intention (i.e., exercise intention). The regulatory fit is captured by the interaction between type of reward (i.e., promotion-type reward vs. prevention-typed reward) and the way the reward is communicated (i.e., via either gain or loss-framed information). The analytical results of the first study reveal a significant fit effect on perceived value, but not on exercise intention. We found that perceived value mediates the impact of the fit effect on exercise intention. In the second study presented in this chapter, we demonstrated that exercise motives (i.e., approach and avoidance) are proximal constructs that mediate the influence of the distal construct of regulatory focus (i.e., promotion and prevention) on reward choice. In chapter 4, we examined the interaction effect between reward type (direct-mail vs. service training program), type of payoff level (low vs. high) and the way payoff is communicated (i.e., either using verbal vs. numerical information) in the context of business-to-business loyalty programs. The results of the first study revealed that the interaction between reward type and type of payoff level is a determinant factor of perceived value and investment decision. The latter two constructs were found to positively influence loyalty. In the second study, we replicated the finding of the first study. In addition, we also demonstrated that the format in which information is presented makes a substantive difference in consumer perceptions toward the value of a reward. In chapter 5, we examined the influence of the fit effect on perceived value of a health plan and insurance purchase decision. The study in this chapter showed that value-from-fit was created as a result of the interaction effect between regulatory focus concerns and the way the message is framed (i.e., gain vs. loss). This study also demonstrated that perceive value mediates the impact of fit effect on purchase intentions. 
Finally, our empirical studies revealed several interesting results. First, fit effect is found to be an influential factor that explains variance in reward perceived value. In chapter 5 , we demonstrate that even in the presence of a strong influence of context-specific determinants of perceived value, the fit effect still plays albeit a small, role, i.e., offering additional explanation of variance in perceived value. Second, we revealed that reward perceived value, in turn, influences investment decision leading to loyalty. This result underscores the significant relationship between attitude and behavioral intention, which is indicative of loyalty (e.g., Cronin, Brady and Hult, 2000). Moreover, this finding confirms previous results in RF research that demonstrate that value created from fit can be transferred to consumer's subsequent evaluations. Third, based on our meta-analysis study, the strength of fit effect is found to vary across research settings. The results of our empirical studies seem to corroborate this finding, as there were differences in the path coefficients associated with the fit effect and as well as in the proportion of variance in the perceived value (R-square) that was accounted for by the fit effect. Therefore, given that fit effect has a significant impact on consumer decision making, therefore, managers should take the fit effect into account in positioning rewards as a strategy for attracting new customers and retaining existing ones.

\section{The Role of Domain Specific Constructs}

Previous RF research has mainly focused on the influence of the fit effect on consumer responses without taking the effect of substantial constructs (e.g., social and socioeconomic factors) into account, which may explain variance in consumer behavior. In fact, the objectives of many RF studies is to show whether there is additional value created from the fit effect, independent of the value inherently derived from the object of decision-making (Higgins et al., 2003). In order to test this, previous research usually compares responses of participants in fit vs. nonfit conditions. For instance, Higgins et al., (2003) found that participants in the fit condition assigned the price to a mug almost $50 \%$ higher than those of participants in nonfit condition. Thus, another contribution of our dissertation is the role of regulatory focus in the presence of domain specific constructs that substantially affect consumer behaviors.

As has been mentioned in the previous chapter, we conclude that there is an influence of the fit effect in the presence of risk perceptions in explaining consumers' perceived value toward a health insurance policy and consumers' purchase intention. From a managerial perspective, this result suggests that the fit effect has an impact on consumer decision making because it relates to the details of an insurance policy.

As described in chapter 3, the influence of RF on consumer behavior within health club loyalty programs may not be direct, but mediated by the domain-specific construct of exercise motives. Specifically, we found that avoidance exercise motives mediate the impact of prevention focus strength on the choice of a prevention reward. In this case, RF serves as 
distal personality characteristic that is stable and its effect on attitudes and behaviors is indirect, whereas exercise motives are proximal constructs, which are specific to situations and are malleable overtime. From a managerial perspective, this result could help managers tailor specific rewards to different LP members that have different goals as their domainspecific motives are likely to predict their behaviors.

\section{Assessing the Simultaneous Impact of Trait RF vs. State RF}

In two studies reported in chapter 4, we assessed the impact of state and trait RF simultaneously on participants' response measures. To the best of our knowledge, so far, there was not one single study in previous RF that examined this issue. In study 1, we found that promotion strength has a significant positive impact on value and investment decisions, whereas prevention strength has a significant negative impact on investment decisions and is insignificant in relation to value. In the study 2 of that chapter, promotion strength was shown to have a positive significant impact on value and investment decision and prevention strength was shown to have a negative significant impact on value and investment decision. Thus, these two studies demonstrated that in addition to the impact to state RF, the trait RF (i.e., either promotion or prevention) may explain additional variances in outcome variables. Our results refute the assumptions frequently made in RF theorizing (e.g., Higgins and Spiegel, 2004), according to which when RF is manipulated situationally, the state RF as a result of the priming would dominate the trait.

\section{Perspective on Future Research}

In addition to specific recommendations for future research provided at the end of each chapter, this final section introduces a number of general directions for future research on regulatory fit.

To account for the underlying mechanism of value creation in a situation in which people experience regulatory fit, three explanations have been advanced in the literature. First, the concept of 'feeling-right' has been well-accepted as one of the underlying mechanisms, namely when fit occurs people will feel right about their choices which in turn, affects their subsequent judgments (e.g., attitude toward an object under evaluation) (Higgins et al., 2003). Second, Lee and Aaker (2004) offered a persuasion-based explanation. They contended that the subjective experience of processing fluency may underlie the fit effect, namely that a high level of fit may lead to more fluent processing of a message, which in turn, influences subsequent evaluations. Their reasoning was that people will digest a message more fluently if the content of the message is consistent with the way they naturally think about the issue. Third, and more recently, the founder of regulatory fit theory, Higgins (2006), advanced the concept of strength of engagement as the explanation of value creation in case people experience fit. According to Higgins (2006), when people experience fit, 
their 'feel right' feeling makes them engage more strongly in what they are doing. Furthermore, the experience during the engagement will create additional value, which later adds more value to the object being pursued. Future research could explore these routes to value creation, for instance, by building an integrative model involving constructs of processing fluency (e.g., the believability of the message), feel right, strength of engagement, and value.

In our empirical studies that combined state and trait RF, we found that trait RF had an additional influence on attitudes and behaviors. This finding suggests that future research could investigate other trait covariates that might influence variance in consumers attitudes and behavior in a situation where state RF is induced. For instance, in a recent novel titled Gost by Robert Harris (p. 85, 2007) it is suggested that: "People who succeed in life are rarely reflective". This seems to reflect the need to control for the personality trait of reflexivity (Tett and Burnett, 2003). This construct refers to an individual's critical evaluation of his/her performance. Thus, the question becomes whether being reflective is more a characteristic of promotion or prevention-focused individuals? This issue is important to consider as members who fail in attaining their goals of getting LP rewards will tend to search for reasons for their failures. Searching for reasons following bad outcomes is in line with the directive function of mood (Schwartz and Clore, 1983) that claims that people in a bad mood tend to sort out the plausible causes of their bad affective state.

Another issue for future research is the dynamics of state regulatory focus. In our empirical studies, we measured and manipulated participants' regulatory focus at one point of time. However, participants' state regulatory focus is transient or may endure only temporarily. Therefore, it is subject to change, depending on which of the types of environmental stimuli that trigger the foci. Loyalty programs are long-term oriented, and hence, multiple purchase decisions are involved over a long period of time. Therefore, members' behaviors may not be persistent over time. Furthermore, redemption decisions in B-to-B loyalty programs can be strongly influenced by the dynamic of the business environment in which a firm operates. Therefore, studying the dynamic of regulatory focus and its influence on consumer's longitudinal behavior is an interesting future research avenue.

Recent research on regulatory focus (Aaker and Lee, 2001) revealed that people in Eastern cultures are generally more prevention oriented, whereas those in Western cultures are more promotion oriented. Therefore, we recommend that future research on regulatory fit should take the cultural background of participants into account and the findings of the research should be generalized across different cultures.

Finally, we hope that this dissertation has contributed to more insights on the effects of regulatory fit and stimulates others to explore and fulfill research gaps that we outlined. 


\section{APPENDICES}

\section{Sample Scenarios}

\section{Appendix A: Chapter 3}

\section{Prevention Reward (dietary advice), Loss Frame}

Please imagine that you have been working out at your health club for some time now. Now, please also imagine that the health club management is about to launch in this month a new loyalty reward program for its members. The loyalty program works as follows: For every visit you use the gym facilities, you will earn one point. If you reach 100 points, you can spend these points on a reward. The type of rewards available can be different every year. In order to participate and benefit from this loyalty program, the gym management requires you to register as a member. If you exercise regularly at least twice a week, within a year, you will get enough credit points.

Now, please also imagine that you are a member of a loyalty program and are interested in earning the freeearned credit points. For the present period, the health club management announces that the free-earned credit points can be traded in for a reward of dietary advice (equivalent to 75 AUD), which consists of a program for measuring your desired weight, measuring your calories to avoid decreased metabolism, and advice and a plan to optimize your diet and food intake. Research in sport psychology has proved that the dietary advice program is necessary to maintain your exercise level. In general, having dietary advice is an efficient way to maintain good health condition.

You don't want to miss out on this reward because you believe that by not having dietary advice, you will fail in achieving your exercise goals. Concerning the amount of credit points you have at the moment, if you don't exercise in the health club for at least two times per week from now on, you will fail to get sufficient amount of credit points at the end of the year. 


\title{
Appendix B.1: Chapter 4 (Study 1)
}

\author{
Consumer direct mail, high payoff, numerical format
}

Please imagine that you have earned 2000 credit-points last year in the channel reward program (CRP) designed by [company $X$ ]. You are now able to invest these 2000 credit-points in sales and service support and it is all your decision. However, your attention is attracted toward a consumer direct mail program, which will support you in improving your promotional strategy in order to attract new customers. This program will help you in approaching prospects twice a year and the administration of the program will be handled by a direct mail consultant. The program is expected to improve your customer database, enhance sales, and enable you to eventually improve your bottom-line results.

The conditions of the program are as follows: You are expected transfer your customer-database to a direct-marketing agency that will treat this information confidentially. This direct mail program will cost you all your 2000 free-earned credit-points.

Of course you are interested in the potential pay-off and improvements of your results if you invest your 2000 credit-points in this direct mail program. The management of company $\mathrm{X}$ informs you that there is an $\mathbf{8 0} \%$ chance that your revenues will be increased by $15 \%$, which gives you an expected payoff of $12 \%$ in case you invest in this reward.

\section{Appendix B.2: Chapter 4 (Study 2)}

\section{Consumer direct mail, high payoff, verbal format}

Please imagine that you have earned 2000 credit-points last year in the channel reward program (CRP) designed by [company $X$ ]. You are now able to invest these 2000 credit-points in sales and service support and it is all your decision. However, your attention is attracted toward a consumer direct mail program, which will support you in improving your promotional strategy in order to attract new customers. This program will help you in approaching prospects twice a year and the administration of the program will be handled by a direct mail consultant. The program is expected to improve your customer database, enhance sales, and enable you to eventually improve your bottom-line results.

The conditions of the program are as follows: You are expected transfer your customer-database to a direct-marketing agency who will treat this information confidentially. This direct mail program will cost you all your 2000 free-earned credit-points.

Of course you are interested in the potential pay-off and improvements of your results if you invest your 2000 credit-points in this direct mail program. The management of company $\mathrm{X}$ informs you that it is quite likely that your revenues will be increased by $15 \%$, which gives you an expected payoff of $1.2 \%$ in case you invest in this reward. 


\section{Appendix B.3: Chapter 4}

Manipulation checks for formats of payoff uncertainty information modes (Study 2)

\section{Numerical format}

Suppose that a $0 \%$ chance of an event to occur means impossible, and $100 \%$ chance means certain. What does the statement that "there is an $80 \%$ [30\%] chance that your revenues will increase", mean to you? (Please tick only one option that mostly applies to you):

\begin{tabular}{ll}
$100 \% \quad$ Certain \\
& Extremely likely \\
& Quite likely \\
& Rather likely \\
& Somewhat likely \\
\hline & As likely as is unlikely \\
\hline & Somewhat unlikely \\
\hline & Rather unlikely \\
\hline & Quite unlikely \\
\hline & Extremely unlikely \\
\hline Impossible
\end{tabular}

\section{Verbal format}

Suppose that, an event that is impossible to occur, is said to have a $0 \%$ chance of occurrence, and an event that is certain to occur is said to have $100 \%$ chance. What does the statement that "it is quite likely [rather unlikely] that your revenues will increase" means to you (please tick only one option that mostly applies to you):

Certain: $100 \%$

90

80

70

60

50

40

30

20

10

Impossible: $0 \%$ 


\title{
Appendix C.1: Chapter 5
}

\author{
Sample Scenarios: Getting a discount and loss-framed message condition
}

Imagine the following situation: At the moment, you have health insurance with insurance company ABC, where you need to pay a monthly premium of $€ 92.50$ with no personal excess. However, you are considering seeking an alternative to your present health insurance to cover your medical expenses.

Please also imagine that you are attracted to a new health insurance policy advertised recently by insurance company XYZ. The company XYZ offers you the following scheme:

You can purchase a policy with a monthly premium of $€ 87.50$ with personal excess of $€ 0$. In addition, company XYZ offers a discount of $€ 15$ in the monthly premium if you take an annual personal excess of $€ 500$. This means that in case you have a health treatment within one year, then you need to pay the first $€ 500$ of the treatment cost.

After making some calculations you realize that your financial situation is quite healthy and that you can cover medical expenses you incur even with a $€ 500$ personal excess. Moreover, your concern is to get the discount.

Now, if you don't choose the company XYZ's alternative, namely, the insurance scheme with a personal excess of $€ 500$, you will not receive a discount of $€ 15$ each month.

\section{Appendix C.2: Chapter 5}

\section{$\underline{\text { Avoiding a cost-sharing and gain-framed message condition }}$}

Imagine the following situation: At the moment, you have health insurance with insurance company $\mathrm{ABC}$, where you need to pay a monthly premium of $€ 87.50$ with no personal excess. However, you are considering seeking an alternative to your present health insurance to cover your medical expenses.

Please also imagine that you are attracted to a new health insurance policy advertised recently by insurance company XYZ. The company XYZ offers you the following scheme:

You can purchase a policy with a cheaper monthly premium of $€ 72.50$. However, if you buy this policy, you have to take an annual personal excess of $€ 500$. This means that in case you have a health treatment within one year, then you need to pay the first $€ 500$ of the treatment cost. If you don't want take to the personal excess, company XYZ will charge a cost-sharing fee of $€ 15$ that will be added to the monthly premium.

After making some calculations, you realize that your financial situation is quite healthy and that you can cover medical expenses you incur even with a $€ 500$ personal excess. Moreover, your concern is to avoid the cost-sharing.

Now, if you choose the company XYZ's alternative, namely, the insurance scheme with a personal excess of $€ 500$, you don't have to pay the cost-sharing of $€ 15$ each month. 


\section{REFERENCES}

References marked with an asterisk indicate studies included in the meta analysis.

\section{A}

Aaker, Jennifer L. and Angela Y. Lee (2001), “'I' Seek Pleasures and 'We' Avoid Pains: The Role of Self-Regulatory Goals in Information Processing and Persuasion.” Journal of Consumer Research, 28 (June), 33-49.

- and (2006), "Understanding Regulatory Fit." Journal of Marketing Research, 43 (February), 15-19.

Avnet, Tamar and E. Tory Higgins (2003), "Locomotion, Assessment, and Regulatory Fit: Value Transfer from "How" to "What"," Journal of Experimental Social Psychology, 39(5), 525-530.

and E. Tory Higgins (2006), "How Regulatory Fit Affects Value in Consumer Choices and Opinions," Journal of Marketing Research, 43 (February), 1-10.

Ajzen, Icek (1991). The Theory of Planned Behavior. Organizational Behavior \& Human Decisions Processes, 50(2), 179-211.

Ajzen, Icek and Martin Fishbein (1980), Understanding attitudes and predicting behavior. Englewood Cliffs, NJ: Prentice-Hall.

Arora, Raj, Charles Stoner and Alisha Arora (2006), "Using Framing and Credibility to Incorporate Exercise and Fitness in Individuals' Lifestyle," Journal of Consumer Marketing, 23 (4/5), 199-207.

\section{B}

Bagozzi, Richard P. and Youjae Yi (1989), "On the Use of Structural Equation Models in Experimental Designs," Journal of Marketing Research, 26(3), 271-284.

Bakker, Frank. M., R.ene C.J.A. van Vliet and Wynand P.M.M van de Ven (2000), "Deductibles in Health Insurance: Can the Actuarially Fair Premium Reduction Exceed the Deductible? "Health Policy, 53, 125-143.

Banks, Sara M., Peter Salovey and Susan Greener (1995), “The Effects of Message Framing on Mammography Utilization,” Health Psychology, 14(2), 178-184.

Baron, Reuben M. and David A. Kenny (1986), “The Moderator-Mediator Variable Distinction in Social Psychological Research: Conceptual, Strategic, and Statistical Considerations," Journal of Personality \& Social Psychology, 51, 1173-1182.

Bijmolt, Tammo H. A. and Rik G. M. Pieters (2001), "Meta-Analysis in Marketing When Studies Contains Multiple Measurements," Marketing Letters, 12(2), 157-169. 
Budescu, David V., Shalva Weinberg, and Thomas S. Wallsten (1988), "Decisions Based on Numerically and Verbally Expressed Uncertainties," Journal of Experimental Psychology: Human Perception and Performance, 14, 281-294.

Briley, Donnel A., and Jennifer L. Aaker (2006), "When Does Culture Matter? Effects of Personal Knowledge on the Correction of Culture-Based Judgments," Journal of Marketing Research, 43, 395-408.*

Brown, Tom J., John C. Mowen, D. Donavan and Jane W. Licata (2002), "The Customer Orientation of Service Workers: Personality Trait Effects on Self-and Supervisor Performance Ratings," Journal of Marketing Research, 39 (February), 110-119.

Breland, B. Tyson and John J. Donovan (2005), "The Role of State Goal Orientation in the Goal Establishment Process," Human Performance, 18 (1), 23-53. .

Brockner, Joel and E. Tory Higgins (2004), "Regulatory Focus Theory: Implications for the Study of Emotions at Work," Organizational Behavior \& Human Decision Processes, 86 (January), 35-66.

C

Camacho, Christopher J., E. Tory Higgins, and Lindsay Luger (2003), "Moral Value Transfer from Regulatory Fit: What Feels Right is Right and What Feels Wrong is Wrong," Journal of Personality and Social Psychology, 84 (3), 498-510.

Capizzi, Michael T. and Rick Ferguson (2005), "Loyalty Trends for the Twenty-First Century," Journal of Consumer Marketing, 22 (2), 72-80.

Cacioppo, John. T., Richard E. Petty, Jeffrey A. Feinstein and W. Blair G. Jarvis (1996), "Dispositional Differences in Cognitive Motivation: The Life and Times of Individuals Varying in Need for Cognition," Psychological Bulletin, 119, 197-253.

Card, David and Alan B. Krueger (1995) "Time-series Minimum-Wage Studies: A MetaAnalysis," The American Economic Review, 85(2):238-243.

Capizzi, Michael T. and Rick Ferguson (2005), "Loyalty Trends for the Twenty-first Century," Journal of Consumer Marketing, 22(2): 72-80.

Cardon, James H. \& Igal Hendel. (2001), "Asymmetric Information in Health Insurance: Evidence from the National Medical Expenditure Survey," RAND Journal of Economics, 32(3), 408-27.

Carver, Charles. S., and Michael F. Scheier (1981). Attention and Self-regulation: A Control-Theory Approach to Human Behavior. New York: Springer- Verlag.

Press.

(1998). On the Self-Regulation of Behavior. New York: Cambridge University of

Cesario, Joseph, Heidi Grant and E. Tory Higgins (2004), "Regulatory Fit and Persuasion: Transfer From "Feeling Right"," Journal of Personality and Social Psychology, 86(3), 388-404.* 
Cohen, Alma \& Liran Einav (2007), "Estimating Risk Preferences from Deductible Choice," American Economic Review, 97(3), 745-788.

Chavez, Linda. (2007). How to cure the health insurance crisis. http://www.townhall.com/columnists/LindaChavez/.

Chakraborty, Goutam, Richard Ettenson and Gary Gaeth (1994), "Consumers Choose Health Insurance," Journal of Health Care Marketing, 14(1), 21-33.

Cohen, Jacob (1973), "Eta-Squared and Partial Eta-Squared in Fixed Factor ANOVA Designs," Educational and Psychological Measurement 33, 107-112.

(1988), Statistical Power Analysis for the Behavioral Sciences (2nd ed.). Mahwah, NJ: Lawrence Erlbaum. (1992). “A Power Primer,” Psychological Bulletin, 112 (1),155-159.

Chen, Gilad, Stanley M. Gully, Jon-Andrew Whiteman, and Robert N. Kilcullen (2000), "Examination of Relationships among Trait-Like Individual Differences, State-Like Individual Differences, and Learning Performance,” Journal of Applied Psychology, 85(6), 835-847.

Chang, Julia (2006), "Rewarding Loyalty," Sales \& Marketing Management, 158 (2), 16.

Chernev, Alexander (2004), "Goal Orientation and Consumer Preference for the Status Quo," Journal of Consumer Research, 31, 557-565.

Chin, Wynne W. (1998), "The Partial Least Squares for Structural Equation Modeling," in Modern Methods for Business Research, G.A. Marcoulides (ed.). Mahwah, NJ: Lawrence Erlbaum Associates, 295-333.

— (2001), PLS-Graph User's Guide Version 3.0. Houston, TX: C.T. Bauer College of Business, University of Houston.

Crowe, Ellen and E. Tory Higgins (1997), "Regulatory Focus and Strategic Inclinations: Promotion and Prevention in Decision-Making." Organizational Behavior \& Human Decision Processes, 69(2), 117-132.

Cronin Jr., J. Joseph, Michael K. Brady and G. Tomas M. Hult (2000), “Assessing the Effects of Quality, Value, and Customer Satisfaction on Consumer Behavioral Intentions in Service Environments," Journal of Retailing, 76(2), 193-218.

Custers, Thomas, Onyebuchi A. Arah and Niek S. Klazinga (2007), "Is There a Business Case for Quality in the Netherlands? A Critical Analysis of the Recent Reforms of the Health Care System,". Health Policy, 82(2), 226-239.

\section{D}

Day, John, Paul Reynolds, and Geoff Lancaster (2006), "Entrepreneurship and the Small to Medium-Sized Enterprise: A Divergent/Convergent Paradox in Thinking Patterns Between Advisers and SME Owners-Managers," Management Decision, 44(5), 581-597.

Dowling, Grahame R. and Mark Uncles (1997), "Do Customer Loyalty Programs Really Work?” Sloan Management Review, 38(4), 71-82. 
Dowling, Grahame R. (2002), “Customer Relationship Management: In B2C Markets, Often Less Is More,” California Management Review, 44 (Spring), 87-104.

E

Eagly, Alice H. and Shelley Chaiken (1993), The Psychology of Attitudes. ForthWorth, TX: Harcourt Brace.

$\mathbf{F}$

Falk, R.F. and N.B. Miller (1992), A Primer for Soft Modeling. Akron, OH: University of Akron Press.

Forster, Jens, Tory E. Higgins and Lorraine Chen Idson (1998), "Approach and avoidance strength during goal attainment: Regulatory focus and the 'goal looms larger' effect," Journal of Personality and Social Psychology, 75(5), 1115-1131.*

, E. Tory Higgins and Amy Taylor Bianco (2003), "Speed/accuracy Decisions in Task Performance: Built-in Trade-Off or Separate Strategic Concerns?” Organizational Behavior and Human Decision Processes, 90, 148 - 164.

, Heidi Grant, Lorraine Chen Idson and E. Tory Higgins (2001), “Success/Failure Feedback, Expectancies, and Approach/Avoidance Motivation: How Regulatory Focus Moderates Classic Relations," Journal of Experimental Social Psychology.37 (3), 253260.*

and E. Tory Higgins (2005), "How Global Versus Local Perception Fits Regulatory Focus," Psychological Science, 16(8), 631-636.*

Florack, Arnd and Martin Scarabis (2006), "How advertising claims affect brand preferences and category-brand associations: The role of regulatory fit," Psychology and Marketing, 23(9), 741-755.*

Freitas, Antonio L., and E. Tory Higgins (2002), "Enjoying Goal-Directed Action," Psychological Science, 13(1), 1-6. *

Fedorikhin, Alexander and Catherine A. Cole (2004), "Mood Effects on Attitudes, Perceived Risk and Choice: Moderators and Mediators." Journal of Consumer Psychology, 14(1/2), 2-12.

Fuhrer, R., M.J. Shipley, J.F. Chastang, A. Schmaus, I. Niedhammer, S.A. Stansfeid, M. Goldberg, and M.G. Marmot (2002), "Socioeconomic Position, Health, and Possible Explanations: A Tale of Two Cohorts," American Journal of Public Health, 92(8), 12901294.

Fornell, Claes and Fred L. Bookstein (1982), "A Comparative Analysis of Two Structural Equation Models: LISREL and PLS Applied to Consumer Exit-Voice Theory," Journal of Marketing Research, 19, 440-452. 
and David F. Larcker (1981), "Evaluating Structural Equation Models with Un-

observable Variables and Measurement Error," Journal of Marketing Research, 18: 3950.

Freitas, Antonio L. and E. Tory Higgins (2002), "Enjoying Goal-Directed Action,” Psychological Science, 13(1), 1-6.

G

Gabel, Jon R. (2004), "Employers' Contradictory Views about Consumer-Driven Health Care,"Medical Benefits, 21(10), 9-9.

Guariglia, Alessandra and Mariacristina Rossi (2004), "Private Medical Insurance and Saving: Evidence from the British Household Panel Survey," Journal of Health Economics, 23, 761-783.

Greß, Stefan (2006), "Regulated Competition in Social Health Insurance: A Three-Country Comparison," International Social Security Review, 59(3), 27-47.

Grosskopf, Brit, Ido Erev, and Eldad Yechiam (2006), "Foregone with the Wind: Indirect Payoff Information and its Implications for Choice," International Journal of Game Theory, 34(2), 285-302.

\section{$\mathbf{H}$}

Halamish, Vered, Nira Liberman, E. Tory Higgins, and Lorraine Chen Idson (2007), "Regulatory Focus Effects on Discounting over Uncertainty for Losses vs. Gains," Journal of Economic Psychology, doi:10.1016/j/joep.2007.09.002.

Harris, Robert (2007), The Ghost, paperback, London: Hutchinson.

Harris, Jennifer and Mark Uncles (2007), "Modeling the Repatronage Behavior of Business Airline Travelers," Journal of Service Research, 9(4), 297-311.

Head, Thomas C., Ricky W. Griffin, Thomas S. Bateman, Lynn Lohman, and Valerie L. Yates (1988), "The Priming Effect in Task Design Research," Journal of Management, 14 (1), 33-39.

Higgins, E. Tory (1987), "Self-Discrepancy: A Theory Relating Self and Affect," Psychological Review, 94, 319-340.

(1996), Knowledge activation: Accessibility, applicability, and salience. In E. T. Higgins and A. W. Kruglanski (Eds.), Social psychology: Handbook of basic principles (pp. 133-168). New York: Guilford.

(1997), "Beyond Pleasure and Pain," American Psychologist, 52(12): 1280-1300. , James Shah and Ronald Friedman (1997), "Emotional Responses to Goal Attainment: Strength of Regulatory Focus as Moderator," Journal of Personality \& Social Psychology, 72, 515-525. 
(2000), "Does Personality Provide Unique Explanations for Behaviour? Personality as Cross-Person Variability in General Principles," European Journal of Personality, 14(January-April): 391-406.

, Ronald S. Friedman, Robert E. Harlow, Lorraine C. Idson, Ozlem N. Ayduk and Amy Taylor (2001), "Achievement Orientations from Subjective Histories of Success: Promotion Pride versus Prevention Pride," European Journal of Social Psychology, 31: 3-23.*

(2002), "How Self-Regulation Creates Distinct Values," Journal of Consumer Psychology, 12(March), 177-191.

, Lorraine Chen Idson, Antonio L. Freitas, Scott Spiegel and Daniel C. Molden (2003), "Transfer of Value From Fit," Journal of Personality and Social Psychology, 84(6), 1140-1153.*

and Scott Spiegel (2004), "Promotion and Prevention Strategies for SelfRegulation: A Motivated Cognition Perspective,” In R. F. Baumeister \& K. D. Vohs (Eds.), Handbook of self-regulation: Research, theory and applications,. 171-187, New York: Guilford Press.

(2005), "Value from Regulatory Fit," Current Directions in Psychological Science, 14(4), 209-213.

(2006), "Value from Hedonic Experience and Engagement," Psychological Review, 113, 439-460.

Hedges, Larry V., and Ingram Olkin (1985), Statistical Methods for Meta-Analysis. New York: Russell Sage Foundation.

and Jack L. Vevea (1998), "Fixed and Random Effects Models in MetaAnalysis," Psychological Methods, 3(4), 486-504.

Hofstede, Geert (1980), Culture's Consequences: International Differences in Work-Related Values. Newbury Park, CA: Sage.

Hulland, John (1999), "Use of Partial Least Squares (PLS) in Strategic Management Research: A Review of Four Recent Studies," Strategic Management Journal, 20 (February), 195-204.

Hullet, Craig R. and Timothy R. Levine (2003), "The Overestimation of Effect Sizes from F Values in Meta-Analysis: The Cause and a Solution," Communication Monograph, $70(1), 52-67$.

Hunter, John E. and Frank L. Schmidt (1999), Methods of Meta-Analysis, $2^{\text {nd }}$, Newbury Park, CA: Sage.

Huang, Chun L. (1993), "Simultaneous-Equation Model for Estimating Consumer Risk Perceptions, Attitudes, and Willingness-to-Pay for Residue-Free Produce," Journal of Consumer Affairs, 27(2), 377-396. 
Idson, Lorraine Chen, Nira Liberman and E. Tory Higgins (2004), "Imagining How You'd Feel: The Role of Motivational Experiences from Regulatory Fit," Personality and Social Psychology Bulletin, 30(7), 926-937. *

Iwasaki, Yoshi and Mark E. Havitz (2004), "Examining Relationships between Leisure Involvement, Psychological Commitment and Loyalty to a Recreation Agency." Journal of Leisure Research, 36(1), 45-72.

\section{J}

Jain, Shailendra Pratap, Nidhi Agrawal and Durairaj Maheswaran (2006), "When More May Be Less: The Effects of Regulatory Focus on Responses to Different Comparative Frames," Journal of Consumer Research, 33(1), 91-98.*

Jones, Lee W., Robert C. Sinclair and Kerry S. Courneya (2003), "The Effects of Source Credibility and Message Framing on Exercise Intentions, Behaviors, and Attitudes: An Integration of the Elaboration Likelihood Model and Prospect Theory," Journal of Applied Social Psychology, 33(1), 179-196.

Johnson Eric J., John Hershey, Jacqueline Meszaros and Howard Kunreuther (1993), "Framing, Probability Distortions, and Insurance Decisions," Journal of Risk and Uncertainty, 7, 35-51.

Johnson, Michael D., Andreas Herrmann and Frank Huber (2006), "The Evolution of Loyalty Intentions," Journal of Marketing, 70(April), 122-132

\section{K}

Kahneman, Daniel and Amos Tversky (1979), "Prospect Theory: An Analysis of Decision under Risk," Econometrica, 47, 263-291. and (1984), "Choices, Values, and Frames," American Psychologist, 39, 341-350.

Kalaian, Hripsime A. and Stephen W. Raudenbush (1996), "Multivariate Mixed Linear Model for Meta-Analysis," Psychological Methods, 1(3), 227-235.

Kanfer, Ruth and Philip L. Ackerman (2000), "Individual Differences in Work Motivation: Further Explorations of a Trait Framework," Applied Psychology: An International Review, 49(3).

Kay, Aaron C., S. Christian Wheeler, John A. Bargh, and Lee Ross (2004), "Material Priming: The Influence of Mundane Physical Objects on Situational Construal and Competitive Behavioral Choice," Organizational Behavior \& Human Decision Processes, 95(January): 83-96. 
Keeney, Ralph L. and Howard Raiffa (1976), Decisions with Multiple Objectives: Preference and Value Tradeoffs. New York: Wiley.

Keppel, Geoffrey and Thomas Wickens (2004), Design and Analysis: A Researcher's Handbook, 4th ed. Englewood Cliffs, NJ: Prentice Hall.

Keller, Punam A (2006), "Regulatory Focus and Efficacy of Health Messages," Journal of Consumer Research, 33(1), p.109-114. *

Keppel, Geoffrey and Thomas Wickens (2004), Design and Analysis: A Researcher's Handbook, 4th ed. Englewood Cliffs, NJ: Prentice Hall.

Kivets, Ran and Itamar Simonson (2002), "Earning the Right to Indulge: Effort as a Determinant of Customer Preferences Toward Frequency Program Rewards," Journal of Marketing Research, 39 (May), 155-70.

— and (2003), "The Effects of Effort and Intrinsic Motivation on Risky Choice," Marketing Science, 22(4), 477-502.

Kim, Yeung Jo (2006), "The Role of Regulatory Focus in Message Framing in Antismoking Advertisements for Adolescents," Journal of Advertising, 35(1), 143-151.*

Kirmani, Amna and Rui Zhu. "Vigilant Against Manipulation: The Effect of Regulatory Focus on the Use of Persuasion Knowledge," Journal of Marketing Research forthcoming.*

Konstantopoulos, Spyros and Larry V. Hedges, (2004), Meta-Analysis in Kaplan, D. (Ed.), Handbook of Quantitative Methodology for the Social Sciences, 281-297. New York: Sage.

Kozlowski, Steve W. J., Stanley M. Gully, Kenneth G. Brown, Eduardo Salas, Eleanor M. Smith, and Earl R. Nason (2001), "Effects of Training Goals and Goal Orientation Traits on Multidimensional Training Outcomes and Performance Adaptability," Organizational Behavior and Human Decision Processes, 85, 1-31.

Kluger, Avraham N., Elena Stephan, Yoav Ganzach, and Meirav Hershkovitza (2004), "The Effect of Regulatory Focus on the Shape of Probability-Weighting Function: Evidence from Across-Modality Matching Method," Organizational Behavior \& Human Decision Processes, 95(1), 20-39.

Kruglanski, Arie W., Erik P. Thompson, E. Tory Higgins, M. Nadir Atash, Antonio Pierro, James Y. Shah and Scott Spiegel (2000), "To Do the Right Thing! or To Just Do It!: Locomotion and Assessment as Distinct Self-Regulatory Imperatives," Journal of Personality and Social Psychology, 79, 793-815.

(2006), "The Nature of Fit and the Origins of "Feeling Right": A Goal-Systemic Perspective," Journal of Marketing Research, 43(1), 11-14.

Kline, Rex B. (1998), Principles and Practice of Structural Equation Modeling. New York: Guilford Press.

Knapp, Peter, D.K Raynor and D.C. Berry (2004), "Comparison of Two Methods of Presenting Risk Information to Patients about the Side Effects of Medicines," Qual Saf Health Care, 13, 176-180. 
Krishnamurthy, Parthasarathy, Patrick Carter, and Edward Blair (2001), "Attribute Framing and Goal Framing Effects in Health Decisions," Organizational Behavior \& Human Decision Processes, 85(2), 382-399.

Kumar, V and Denish Shah (2004), "Building and Sustaining Profitable Customer Loyalty for the 21 st Century," Journal of Retailing, 80(4), 317-330.

$\mathbf{L}$

Lee, Angela Y. and Jennifer L. Aaker (2004), "Bringing the Frame into Focus: The Influence of Regulatory Fit on Processing Fluency and Persuasion," Journal of Personality and Social Psychology, 86(2), 205-218.*

Leenheer, Jorna, Harald J. van Heerde, Tammo H.A. Bijmolt, and Ale Smidts (2007), "Do Loyalty Programs Really Enhance Behavioral Loyalty? An Empirical Analysis Accounting for Self-Selecting Members," International Journal of Research in Marketing, 24, 31-47.

Leung, Chi-Mei and Shui-Fong Lam (2003), "The Effects of Regulatory Focus on Teachers' Classroom Management Strategies and Emotional Consequences," Contemporary Educational Psychology, 28, 114-125.

Levin, Irwin P.; Sandra L. Schneider, and Gary J. Gaeth (1998), “All Frames Are Not Created Equal: A Typology and Critical Analysis of Framing Effects." Organizational Behavior \& Human Decision Processes, 76(2), 149-188.

Lewis, Michael (2004), "The Influence of Loyalty Programs and Short-Term Promotions on Customer Retention," Journal of Marketing Research, 41 (August), 281-92.

Liberman, Nira, Lorraine Chen Idson, Christopher J. Camacho, and E. Tory Higgins (1999), "Promotion and Prevention Choices between Stability and Change," Journal of Personality \& Social Psychology, 77 (June), 1135-1145.

Lipsey, Mark W., and David. B. Wilson (2001), Practical Meta-Analysis. Thousand Oaks, Calif: Sage Publications.

Liu, Yuping (2007), "The Long-Term Impact of Loyalty Programs on Consumer Purchase Behavior and Loyalty," Journal of Marketing, 71(4), 19-35.

Lockwood, Penelope, Christian H. Jordan, and Ziva Kunda (2002), "Motivation by Positive or Negative Role Models: Regulatory Focus Determines Who Will Best Inspire Us," Journal of Personality \& Social Psychology, 83 (April), 854-864.

Longi, Simonetta, Peter Nijkamp and Jacques Poot (2005), "A Meta-Analytic Assessment of the Effect of Immigration on Wages," Journal of Economic Surveys, 19(3), 451-477.

Loro, Laura (1998), "Loyalty Programs Paying Off for B-to-B," Advertising Age's Business Marketing, 83 (9), 1- 2.

Louro, Maria J., Rik Pieters and Marcel Zeelenberg (2005), "Negative Returns on Positive Emotions: The Influence of Pride and Self-Regulatory Goals on Repurchase Decisions," Journal of Consumer Research, 31(4), 833-840.* 
Lueck, Sarah (2005), "Details of Medicare Drug Plan are Released," Wall Street Journal Eastern Edition, 246(67), A4.

\section{$\mathbf{M}$}

Mackenzie, Scott B. (2001), "Opportunities for Improving Consumer Research through Latent Variable Structural Equation Modeling," Journal of Consumer Research, 28 (January), 159-166.

MacKinnon, David P., Chondra M. Lockwood and Jason Williams (2004), "Confidence Limits for the Indirect Effect: Distribution of the Product and Resampling Methods," Multivariate Behavioral Research, 39 (1), 99-128.

Mallinckrodt, Brent, W. Todd Abraham, Meifen Wei and Daniel W. Russell (2006), "Advances in Testing the Statistical Significance of Mediation Effects," Journal of Counseling Psychology, 53(3), 372-378.

Markland, David and David K. Ingledew (1997), “The Measurement of Exercise Motives: Factorial Validity and Invariance across Gender of a Revised Exercise Motivations Inventory," British Journal of Health Psychology, (February), 361-376.

Maarse, Hans and Yvette Bartholomée (2007), "A Public-Private Analysis of the New Dutch Health Insurance System," The European Journal of Health Economics, 8(1), 7782.

Marquis, M. Susan, Melinda Beeuwkes Buntin, José J. Escarce, Kanika Kapur, Thomas A. Louis and Jill M. Yegian (2006), "Consumer Decision Making in the Individual Health Insurance Market," Health Affairs Web Exclusives, 25, W226-W234.

Martin, R. M., F.L. Marcuse (1958), "Characteristics of Volunteers and Nonvolunteers in Psychological Experimentation," Journal of Consulting Psychology, 22(6), 475-479.

Meyerowitz, Beth E. and Selly Chaiken (1987), "The Effect of Message Framing on Breast Self-Examination Attitudes, Intentions, and Behavior," Journal of Personality and Social Psychology, 52(3), 500-510.

Mischel, Walter (1999), "Implications of Person-Situation Interaction: Getting over the Field's Borderline Personality Disorder," European Journal of Personality, 13, 455-461. (2004), "Toward an Integrative Science of the Person," Annual Review of Psychology, 55(1), 1-22.

Morais, Duarte B., Michael J. Dorsch, and Sheila J. Backman (2004), "Can Tourism Providers Buy Their Customers' Loyalty? Examining the Influence of Customer-Provider Investments on Loyalty," Journal of Travel Research, 42(February): 235-243.

Mourali, Mehdi, Ulf Bockenholt, and Michel Laroche (2007), "Compromise and Attraction Effects under Prevention and Promotion Motivations," Journal of Consumer Research, 34 (2), 234-247.

Muthen, B.O. (1984), "A General Structural Equation Model with Dichotomous, Ordered Categorical and Continuous Latent Indicators," Psychometrika, 49, 115-132. 
McGovern, Gail and Youngme Moon (2007), "Companies and the Customers Who Hate Them," Harvard Business Review, 85(6), 78-84.

McKee, Steve (2007), “The Problem with Loyalty Programs,” BusinessWeek Online, 16-16.

McMullan, Rosalind and Audrey Gilmore (2003), "The Conceptual Development of Customer Loyalty Measurement: A Proposed Scale,” Journal of Targeting, Measurement \& Analysis for Marketing, 11(March): 230-243.

McGregor, Sue L.T. (2007), "International Journal of Consumer Studies: decade review (1997-2006)," International Journal of Consumer Studies, 31(1), 2-18.

$\mathbf{N}$

Nunes, Joseph C. and Xavier Dréze (2006), "Your Loyalty Program Is Betraying You," Harvard Business Review, 84(4), 124-131.

Nunnally, J.C. and I.H. Bernstein (1994), Psychometric Theory, 3d ed. New York: McGraw-Hill.

\section{O}

O'Grady, Kevin E. and Deborah R. Medoff (1988), "Categorical Variables in Multiple Regression: Some Cautions,” Multivariate Behavioral Research, 23(2), 243-260.

\section{P}

Pauly, Mark V. (2007). Risks and benefits in health care: the view from economics. Health Affairs, 26(3), 653-662.

Pauly, Mark V. and Bradley Herring (2007), "Risk Pooling and Regulation: Policy and Reality in Today's Individual Health Insurance Market," Health Affairs, 26(3), 770-779.

Pauly, Mark V., Bradley Herring and David Song (2006), "Information technology and Consumer Search for Health Insurance," International Journal of the Economics of Business, 13(1), 45-63.

Pedhazur, Elazar J. (1997), Multiple Regression in Behavioral Research: Explanation and Prediction, 3d ed. Fort Worth, TX: Harcourt Brace College Publishers.

Pennings, Joost M. E. and Ale Smidts (2000), “Assessing the Construct Validity of Risk Attitude," Management Science, 46(10), 1337-1348.

Pennington, Ginger L. and Neal J. Roese (2003), "Regulatory Focus and Temporal Perspective," Journal of Experimental Social Psychology, 39, 563-576.

Peterson, Robert A. (2001), "On the Use of College Students in Social Science Research: Insights from a Second-Order Meta-Analysis," Journal of Consumer Research, 28 (3), 450-461. 
Perdue, Barbara C. and John O. Summers (1986), "Checking the Success of Manipulations in Marketing Experiments," Journal of Marketing Research, 23(4), 317-326.

Petrick, James F. and Sheila J. Backman (2002), “An Examination of the Construct of Perceived Value for the Prediction of Golf Travelers' Intentions to Revisit," Journal of Travel Research, 41(1), 38-45.

Podsakoff, Philip. M., Scott B. MacKenzie, Jeong-Yeon Lee and Nathan Podsakoff (2003), "Common Method Biases in Behavioral Research: A Critical Review of the Literature and Recommended Remedies," Journal of Applied Psychology, 88(5), 879-903.

\section{$\mathbf{R}$}

Raudenbush, Stephen W. (1994), "Random Effects Models," In H. Cooper and L. Hedges (Eds), The Handbook of Research Synthesis, 301-321, New York: Russell Sage Foundation.

Reinartz, Werner J. and V. Kumar (2000), "On the Profitability of Longlife Customers in a Noncontractual Setting: An Empirical Investigation and Implications for Marketing," Journal of Marketing, 64 (October), 17-35.

Rejda, George E. (1998). Principles of Risk Management and Insurance (6th ed). Boston: Addison-Wesley.

Roese, Neal J., Taekyun Hur, and Ginger L. Pennington (1999), “Counterfactual Thinking and Regulatory Focus: Implications for Action versus Inaction and Sufficiency versus Necessity," Journal of Personality and Social Psychology, 77(6), 1109-1120.

Rosenthal, Robert (1979), "The "File Drawer Problem" and Tolerance for Null Results," Psychological Bulletin, 86, 638-641.

and Donald D. Rubin (1986), "Meta-Analytic Procedures for Combining Studies with Multiple Effect Sizes," Psychological Bulletin, 99, 400-406.

, Ralp L. Rosnow and Donald D. Rubin (2000), Contrasts and Effect Sizes in Behavioral Research: A Correlational Approach. Cambridge: Cambridge Univ. Press.

and M. R. DiMatteo (2001), "Meta-Analysis: Recent Developments in Quantitative Methods for Literature Reviews," Annu. Rev. Psychol, 52, 59-82.

Rothman, Alexander J., Roger D. Bartels, John Wlaschin, and Peter Salovey (2006), “The Strategic Use of Gain- and Loss-Framed Messages to Promote Healthy Behavior: How Theory Can Inform Practice,” Journal of Communication, 56, S202-S220.

_ and Peter Salovey (1997), "Shaping Perceptions to Motivate Healthy Behavior: The Role of Message Framing," Psychological Bulletin, 121(1), 3-19.

Rothschild, Michael L. and William C. Gaidis (1981), "Behavioral Learning Theory: Its Relevance to Marketing and Promotions," Journal of Marketing, 45(Spring), 70-78.

Rust, Roland and Bruce Cooil (1994), "Reliability Measures for Qualitative Data: Theory and Implications," Journal of Marketing Research, 31(February), 1-14. 
Saxe, Robert and Barton A. Weitz (1982), "The SOCO Scale: A Measure of the Customer Orientation of Salespeople," Journal of Marketing Research, 19(August), 343-351.

Semin Gun R., Tory Higgins and Lorena Gil de Montes (2005), "Linguistic Signatures of Regulatory Focus: How Abstraction Fits Promotion More Than Prevention," Journal of Personality and Social Psychology, 89(1), 36-45. *

Simonson, Itamar (2005), “,”In Defense of Consciousness: The Role of Conscious and Unconscious Inputs in Consumer Choice. Journal of Consumer Psychology, 15(3), 211217.

Sirdeshmukh, Deepak, Jagdip Singh, and Barry Sabol (2002), “Consumer Trust, Value, and Loyalty in Relational Exchanges," Journal of Marketing, 66 (January), 15-37.

Sirgy, Joseph M., Stephan Grzeskowiak, and Chenting Su (2005), "Explaining Housing Preference and Choice: The Role of Self-Congruity and Functional Congruity," Journal of Housing and the Built Environment, 20(4), 320-347.

Schwartz, Jeffrey, Ed Scannell, and Christina McEachern (2006), "In Your Own Words: VARs Reveal the Pros and Cons of Channel Programs," Var Business, 22 (6), 66.

Schwarz, Norbert and Gerald L. Clore (1983), "Mood, Misattribution, and Judgments of Well-being: Informative and Directive Functions of Affective States," Journal of Personality and Social Psychology, 45, 513-523.

Schoemaker, Paul J. H. and Howard C. Kunreuther (1979), “An Experimental Study of Insurance Decisions," Journal of Risk \& Insurance, 46(4), 603-618.

Shadish, William R., and C. Keith Haddock (1994), "Combining Estimates of Effect Size," In H. Cooper and L. Hedges (Eds), The Handbook of Research Synthesis, 261-281, New York: Russell Sage Foundation.

Sharp, Elizabeth C., Luc G. Pelletier and Chantal Lévesque (2006), "The Double-Edged Sword of Rewards for Participation in Psychology Experiments," Canadian Journal of Behavioural Science, 38(3), 269-277.

Shugan, Steven M. (2005), "Brand Loyalty Program: Are They Shams?” Marketing Science, 24 (Spring), 185-93.

Stajkovic, Alexander D (1999), "Fitting Parametric Fixed Effect Categorical Models to Effect Sizes: A Neglected Meta-Analytic Approach in Organizational Studies," Organizational Research Methods, 2(1), 88-102.

Steyer, Rolf, Manfred Schmitt and Michael Eid (1999), "Latent State-Trait Theory and Research in Personality and Individual Differences," European Journal of Personality, 13, 389-408.

Shrout, Patrick E. and Niall Bolger (2002), "Mediation in Experimental and Nonexperimental Studies: New procedures and recommendations," Psychological Methods, 7(4), 422-445.

Spiegel, Scott, Heidi Grant-Pillow and E. Tory Higgins (2004), "How Regulatory Fit Enhances Motivational Strength during Goal Pursuit," European Journal of Social Psychology, 34, 39-54. 
Taylor, Gail Ayala and Scott A. Neslin (2005), "The Current and Future Sales Impact of a Retail Frequency Reward Program," Journal of Retailing, 81 (April), 293-305.

Tenenhaus, Michel, Vincenzo Esposito Vinzi, Yves-Marie Chatelinc and Carlo Lauro (2005), "PLS Path Modeling." Computational Statistics \& Data Analysis, 48, 159-205.

Tett, Robert P. and Dawn D. Burnett (2003), "A Personality Trait-Based Interactionist Model of Job Performance," Journal of Applied Psychology, 88(3), 500-517.

Tock, Ed (2006), "More Retention Equals More Referrals and Profits," Fitness Business Pro, 22(6), 30.

Townsend, Ellen and Scott Campbell (2004), "Psychological Determinants of Willingness

to Taste and Purchase Genetically Modified Food," Risk Analysis: An International Journal, 24(5), 1385-1393.

Thompson, Simon G., and Julian P. T. Higgins (2002), "How Should Meta-Regression Analyses Be Undertaken And Interpreted?" Statistics in Medicine, 21, 1559-1573.

Tversky, Amos and Daniel Kahneman (1991), "Loss Aversion in Riskless Choice: A Reference-Dependent Model,” Quarterly Journal of Economics, 106(April), 1039-1061.

$\mathbf{U}$

Uncles, Mark D., Grahame R. Dowling and Kathy Hammond (2003), "Customer loyalty and customer loyalty programs," Journal of Consumer Marketing, 20(4), 294-316.

V

Vohs, Kathleen D. and Roy F. Baumeister (2004), Handbook of Self-Regulation, London: Guilford-Press.

von Winterfeldt, D. and W. Edwards (1986), Decision Analysis and Behavioral Research. Cambridge: Cambridge University Press.

W

Wang, Jing and Angela Y. Lee (2006), "The Role of Regulatory Focus in Preference Construction," Journal of Marketing Research, 43 (February), 28-38.*

Werth, Lioba and Jens Foerster (2007), "How Regulatory Focus Influences Consumer Behavior," Eur. J. Soc. Psychology, 37, 33-51.*

Windschilt, Paul D. and Gary L. Wells (1996), "Measuring Psychological Uncertainty: Verbal versus Numeric Methods," Journal of Experimental Psychology: Applied, 2 (4), 343-364. 
Wold, Herman (2001), "Partial Least Squares," in Encyclopedia of Statistical Sciences, Vol. 6, S. Kotz and N.L. Johnson, eds. New York: Wiley, 581-591.

Worth, Keilah A., Helen W. Sullivan, Andrew W. Hertel, Robert W. Jeffery and Alexander J. Rothman (2005), "Avoidance Goals Can Be Beneficial: A Look at Smoking Cessation,” Basic \& Applied Social Psychology, 27(2), 107-116.

Wiener, Norbert (1948), Cybernetics or Control and Communication in the Animal and the Machine, Paris, Hermann et Cie, Cambridge: MIT Press.

Wirtz, Jochen; Anna S. Mattila and May Oo Lwin (2007), "How Effective Are Loyalty Reward Programs in Driving Share of Wallet?" Journal of Service Research, 9(4), 327334.

Wu, George, Jiao Zhang, and Richard Gonzales (2007), "Decision under Risk," in The Blackwell Handbook of Judgment and Decision Making, D. Kohler and N. Harvey, eds. Cambridge: Oxford University Press, 399-423.

$\mathbf{Y}$

Yi, Youjae and Hoseong Jeon (2003), "Effects of Loyalty Programs on Value Perception, Program Loyalty, and Brand Loyalty," Journal of the Academy of Marketing Science, 31(March): 229-240.

$\mathbf{Z}$

Zhao, Guanzhi and Cornelia Pechmann (2007), "The Impact of Regulatory Focus on Adolescents' Response to Antismoking Advertising Campaigns," Journal of Marketing Research Article Preprint, 44, 1-46.*

Zhou, Rongrong and Michel Tuan Pham (2004), "Promotion and Prevention across Mental Accounts: When Financial Products Dictate Consumers' Investment Goals," Journal of Consumer Research, 31 (February): 125-135. 


\section{ABOUT THE AUTHOR}

Ahmad Daryanto was born on 16 June 1973 in Padang, West Sumatera, Indonesia. After completion of his senior high school (SMA 2, Padang) in 1992, he went to Bandung, a capital city of the province of West Java, to pursue his university education at the Institute of Technology Bandung (ITB). He studied Environmental Engineering. While at ITB, he was selected to represent West Java as one of the Indonesian students to follow Youth Exchange Program, which was organized by the Indonesian Ministry of Education together with Canada World Youth. During this program, from August 1996 to March 1997, he was in Canada, and Aceh, Indonesia. On October 1997, he graduated from ITB. In the following year, he received a full scholarship from the Confederation of the Netherlands Industry and Employers (NVO-NCW) to pursue a master degree in Engineering Mathematics at the Faculty of Mathematical Sciences, University of Twente, the Netherlands. He graduated in June 2000 with the MSc final project 'Approximation of the Response Time Distribution in Multi-Class Queueing Networks with Special Structures' that was carried out at the Department of Network Planning at KPN Research at Leidschendam, the Netherlands. In March 2004, he started his PhD in Marketing at the Maastricht Universiteit, Maastricht, the Netherlands. During his time as a $\mathrm{PhD}$ candidate, he was actively involved in the smallgroup teaching method at a wide variety of entry-level and more advanced marketing courses. In addition, he has successfully supervised a substantial number of Master theses. On 30 April 2005, he met Rilda Aprisanti Oelangan Taneko in Maastricht, who was studying for a master degree in Gender and Development studies at the Institute of Social Studies (ISS), in the Hague, the Netherlands. They got married and are blessed with a lovely son Ilham Idraki Ahmad, who was born in Maastricht. 